SERVIÇO DE PÓS-GRADUAÇÃO DO ICMC-USP

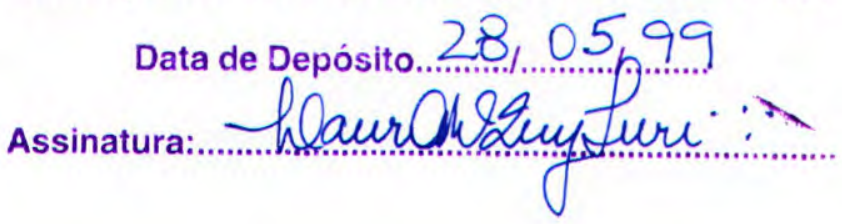

\title{
Inferência Bayesiana para Dados \\ Clínicos Exponenciais com Variáveis Auxiliares
}

\author{
Josenildo de Souza Chaves \\ Orientador : Prof ${ }^{0}$. Dr. Josemar Rodrigues
}

Dissertação apresentada ao Instituto de Ciências Matemáticas e de Computação - USP, como parte dos requisitos para a obtenção do título de Mestre em Ciências - Área de Ciências de Computação e Matemática Computacional.

USP - São Carlos

Julho de 1999. 
Aos meus pais, José e Branca e a minha esposa Eleni. 


\section{Agradecimentos}

Agradeço a Deus, que me conduziu firme durante toda esta jornada.

Desejo também agradecer a muitas pessoas, as quais de alguma maneira contribuíram para que este trabalho fosse realizado.

Ao Professor Dr. Josemar Rodrigues pela orientação segura e dedicada, pela ativa participação e pelo incentivo durante toda a pesquisa.

Ao Professor Dr. Jorge Alberto Achcar e ao Professor Dr. José Galvão Leite pelas valiosas sugestões e comentários decorrentes do exame de qualificação.

Ao Professor Dr. Marinho Gomes de Andrade Filho pelo incentivo, sugestões e pela amizade.

Aos meus amigos do Curso: Viviane Fortulan, Sandra Oliveira, Márcia Mizoi, Silvana Meira, Selene Loibel, Ana Cláudia Melo, Cláudia Hutter, Vanda Donizete, Daniela Brassolatti, Daniela Barattela, Lucimary Santos, Leonilce, Valéria, Cillene, Lillian Kátia, Luciana, Patrícia Leite, Maristela, Cristina Endo, Luciane, Marcos Araújo, Juan Cid, Ulisses dos Anjos, José Roberto Temponi, Gilberto Rinaldi, Gilberto Pereira, Vanderly Janeiro, Luciano Barbosa, Sadao, Donato, Willian, Emerson, Juliano.

Aos funcionários do ICMC, especialmente aos da pós-graduação, a Gláucia Cristianini e a Rosemeire Zambon, a Bhety, a Laura e a Marília.

À Vera Tomazella, pelo incentivo e apoio, desde a minha vinda e permanência na Cidade de São Carlos. 
À Eleni Andrade, pela paciência, carinho, e incentivo em momentos importantes que passamos.

À minha família pelo apoio e pelo incentivo neste período de muitas ausências.

Aos meus colegas professores e funcionários do Departamento de Matemática da UFMA, pelo constante incentivo que tenho recebido.

À CAPES pelo apoio financeiro.

Enfim, gostaria de agradecer a todos que direta ou indiretamente contribuíram para que este trabalho fosse realizado.

"De tudo restaram três coisas:

a certeza de que estava sempre começando, a certeza de que era preciso continuar e a incerteza de que não seria interrompido antes de terminar.

Fazer da interrupção um caminho novo,

fazer da queda um passo de dança, do medo uma escada, do sonho, uma ponte, da procura, um encontro". 


\section{ÍNDICE}

Capítulo 1 - Introdução ….............................................................................................. 1

1.1 Revisão Bibliográfica .............................................................................................. 2

1.2 Objetivos Principais .........................................................................................

1.3 Apresentação dos Capítulos.......................................................................................

Capítulo 2 - Dados Clínicos Exponenciais - Abordagem Clássica ............... 5

2.1 Modelo Estatístico sem Censuras .................................................................................. 5

2.1.1 Ilustrações Numéricas e Gráficas .............................................................. $\quad 10$

2.2 Modelo Estatístico com Censuras ............................................................................ 14

2.2.1 Variâncias Assintóticas das Estimativas de a e b ............................................ 17

2.2.2 Todos os Pacientes Entram Juntos no Estudo .............................................. 18

2.2.3 Entrada Uniforme no Estudo ................................................................... 19

2.3 Modelos Estatísticos Relacionados a Várias Variáveis Auxiliares .............................. 20

2.3.1 Modelo Estatístico sem Censuras ................................................................. 20

2.3.2 Modelo Estatístico com Censuras ..................................................................... 21

Capítulo 3 - Dados Clínicos Exponenciais - Abordagem Bayesiana

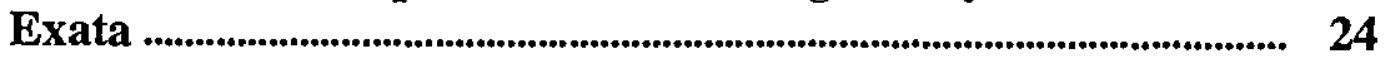

3.1 Modelo Estatístico com Censuras do Tipo III ……................................................... 24

3.2 Densidades a Priori ............................................................................................ 25

3.3 Estimação Bayesiana para a (DPG) ...................................................................... 26

3.4 Estimação Bayesiana para Amostras com Censuras do Tipo III ................................... 28

3.5 Ilustrações Numéricas e Gráficas ............................................................................. 30

Capítulo 4 - Funções de Ligações Alternativas ................................................... 34

4.1 Abordagem Clássica …….............................................................................. 34

4.2 Abordagem Bayesiana ................................................................................... 36

Capítulo 5 - Inferência Bayesiana com Densidades a Priori Obtidas Através das Variáveis Auxiliares ......................................................... 40

5.1 Priori com Médias Condicionais e Priori com Dados Ampliados .............................. 40

5.2 Modelos Lineares Generalizados ........................................................................ 41 
5.3 Especificação da Priori

5.4 Procedimento Bayesiano para um Modelo de Regressão Exponencial com uma Variável Auxiliar

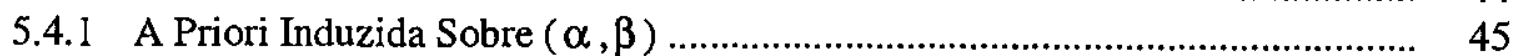

5.4.2 Densidades a Posteriori Conjuntas e Marginais ............................................. 47

Capítulo 6 - Verificação da Adequação de Modelos e Análise de Sensibilidade

6.1 Um Procedimento para Verificação da Adequação de Modelos - Nível

Descritivo Bayesiano $\left(n_{d}\right)$

6.2 Análise de Sensibilidade

Capítulo 7 - Regressão Exponencial Bayesiana com uma Variável Auxiliar - Aplicações

7.1 Inferência para a Função de Ligação Logarítimica ................................................ 52

7.1.1 Modelo com Dados Censurados e Priori PDA ….......................................... 53

7.1.2 Modelo com Dados Completos e Priori PDA …............................................ 56

7.1.3 Comparando o Efeito entre a Amostra Completa e Censurada ....................... 58

7.1.4 Modelo com Dados Censurados e Priori Não-Informativa Difusa .................. 61

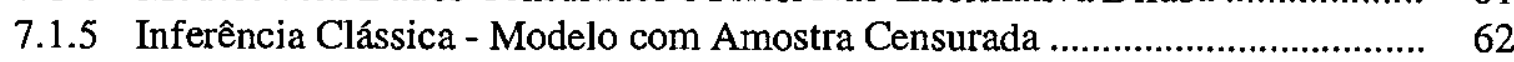

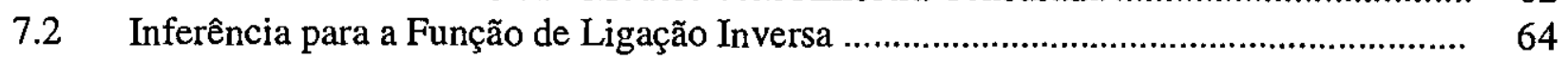

7.2.1 Modelo com Dados Censurados e Priori PDA …........................................... 64

7.2.2 Modelo com Dados Censurados e Priori Gama .................................................. 66

7.3 Inferência para a Função de Ligação Linear ............................................................. 68

7.3.1 Modelo com Dados Censurados e Priori PDA ….............................................. 68

Capítulo 8 - Conclusões Finais e Propostas Futuras …............................................ 71

Apêndice A - Integrais Utilizadas na Inferência Bayesiana Exata ................... 72

Apêndice B - Simulação Monte Carlo em Cadeia de Markov ............................. 74

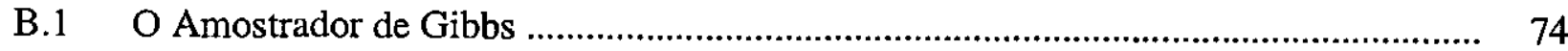

B.2 Algoritmo de Metropolis-Hastings ................................................................. 75

Apêndice C - Alguns Programas Desenvolvidos ........................................................ 78

Referências Bibliográficas ............................................................................................ 93 


\section{Resumo}

Apresentamos neste trabalho, uma análise bayesiana para dados clínicos exponenciais com variáveis auxiliares. Formulamos uma abordagem bayesiana com densidades a priori informativas, obtidas através das variáveis auxiliares sob o contexto de modelos lineares generalizados, para estimar os parâmetros de interesse, testar o modelo e prever a sobrevivência de pacientes com doenças graves. Diferentes funções de ligações são consideradas. $O$ método que iremos examinar consiste na obtenção de informações a priori para a média das respostas, com correspondentes variáveis auxiliares fixas de modo que se possa induzir uma distribuição a priori sobre os coeficientes de regressão a partir de médias condicionais a priori. Esta abordagem utiliza os algoritmos computacionais do tipo Gibbs Sampling/Metropolis-Hastings e será comparada com a inferência bayesiana exata. Finalizamos com aplicações em dados clínicos exponenciais para pacientes com leucemia utilizando amostras completas e amostras censuradas. 


\section{Abstract}

In this work, a Bayesian analysis for the exponential clinical data with auxiliary variables is presented. This Bayesian approach, with informative priors obtained under the context of generalized linear models with fixed auxiliary variables, is formulated to estimate the parameters of interest, to test models and to predict the survival time of patients with serious diseases. Various link functions are considered. The method that we are going to study consists in obtaining prior information for the response mean corresponding to observable variables with fixed covariates, such that we are able to induce a prior distribution on the regression coefficients. This approach uses Gibbs Sampling/Metropolis-Hastings algorithms and it will be compared with the exact Bayesian inference. We end with applications based on censored and uncensored exponential clinical data for patients with leukemia diseases. 


\section{Capítulo 1}

\section{INTRODUÇÃO}

Informações clínicas disponíveis para um médico no instante do diagnóstico de um câncer ou de outra doença são utilizadas para previsão do tempo de sobrevivência, escolher o tratamento para um dado paciente, e para muitos outros objetivos. Os dados podem provir da história médica, de exames físicos, ou de estudos de laboratório. Tais informações podem ser chamadas de informações concomitantes, covariadas ou variáveis auxiliares e têm sido bastante usadas de modo intuitivo. Estamos interessados em desenvolver modelos estatísticos que possam prever o tempo de sobrevivência usando variáveis auxiliares. Este último termo é o que será utilizado ao longo deste trabalho.

A escolha de um modelo apropriado envolve várias considerações: conceito de simplicidade, tratabilidade matemática, adequação do ajuste aos dados e fácil interpretação. Existem ao menos duas principais razões pelas quais um modelo estatístico de sobrevivência baseado em variáveis auxiliares é desenvolvido: (i) prever a sobrevivência de pacientes com uma particular combinação de variáveis auxiliares; (ii) avaliar a importância relativa de tais combinações na previsão do tempo de sobrevivência.

A distribuição do tempo de sobrevivência é geralmente caracterizada por três funções: (1) a função de sobrevivência, (2) a função de densidade de probabilidade, e (3) a função de risco. Estas três funções são matematicamente equivalentes, se uma delas é dada, as outras duas podem ser derivadas.

O tempo de sobrevivência de um paciente com doença crônica em geral é obtido como o tempo entre o diagnóstico ou o início de um tratamento específico e a morte. Geralmente, existem fatores (informação ou variável auxiliar) que explicam a gravidade da doença no instante em que o paciente faz o seu primeiro diagnóstico ou inicia o tratamento. Por exemplo, em pacientes com leucemia a quantidade de glóbulos brancos no instante do diagnóstico é uma 
informação adicional extremamente importante para prever a sua sobrevivência. A leucemia é um câncer cuja gravidade está associada a alta quantidade de glóbulos brancos no sangue. Qualquer modelo estatístico para prever a sobrevivência de um paciente para ser realístico deverá refletir este tipo de dependência entre o tempo de sobrevivência e a variável auxiliar.

\subsection{REVISÃO BIBLIOGRÁFICA}

Estudos da função de sobrevivência em vários ensaios clínicos envolvendo pacientes com câncer em estágio avançado (Prentice, 1973) têm sugerido a função de sobrevivência exponencial. Nestes estudos, variáveis auxiliares, tais como causas da doença, estágio da doença são registradas quando o paciente entra no estudo. Quando estas variáveis são importantes, várias funções de ligações podem ser propostas

Em geral, um modelo exponencial para relacionar a dependência entre o tempo de sobrevivência e a variável auxiliar é utilizado da seguinte forma:

Seja $t_{1}, t_{2}, \ldots, t_{n}$, uma amostra de $n$ independentes tempos de sobrevivência de pacientes, onde o tempo de sobrevivência do i-ésimo paciente possui a seguinte função de densidade:

$$
\begin{array}{ll}
\mathrm{f}_{\mathrm{i}}\left(\mathrm{t} \mid \mu_{\mathrm{i}}\right)=\frac{1}{\mu_{\mathrm{i}}} \exp \left\{-\frac{\mathrm{t}}{\mu_{\mathrm{i}}}\right\}, & \mathrm{t}_{\mathrm{i}}>0, \mu_{\mathrm{i}}>0 .
\end{array}
$$

onde $g\left(\mu_{\mathrm{i}}\right)=\eta_{\mathrm{i}}$ é uma função de ligação (conhecida) entre o tempo médio de sobrevivência e o preditor linear $\eta_{\mathrm{i}}=\mathrm{a}+\mathrm{bx}_{\mathrm{i}}$, com a e $\mathrm{b}$ representando os parâmetros desconhecidos do modelo e $\mathrm{x}_{\mathrm{i}}$ a variável auxiliar. Na literatura, este tipo de modelo é conhecido como Modelo Linear Generalizado (MLG), ver por exemplo Nelder e Wedderburn (1972), onde as seguintes funções de ligações são utilizadas : 


$$
g\left(\mu_{\mathrm{i}}\right)= \begin{cases}\ln \left(\mu_{\mathrm{i}}\right) & \text { :função logarítmica } \\ \mu_{\mathrm{i}} & \text { :função linear } \\ \frac{1}{\mu_{\mathrm{i}}} & \text { :função inversa }\end{cases}
$$

Feigl e Zelen (1965) desenvolveram para as funções de ligações acima, uma teoria de verossimilhança assintótica para estimar os parâmetros a e b quando os dados são não censurados, isto é, todos os pacientes morreram durante o experimento. Zippin e Armitage (1966), supondo função de ligação do tipo linear, generalizaram o trabalho de Feigl e Zelen para pacientes censurados, isto é, alguns pacientes estavam vivos quando o experimento terminou. Byar et al (1974), também propõe uma generalização para dados censurados e função de ligação inversa com várias variáveis auxiliares. Bhattacharya et al (1995) introduziram uma abordagem bayesiana exata, mas extremamente complexa do ponto de vista algébrico, supondo função de ligação do tipo inversa e dados censurados.

Mais recentemente, Bedrick et al (1996) sob função de ligação logarítmica, utilizaram distribuições a priori informativas, extraídas a partir de médias condicionais a priori.

\subsection{OBJETIVOS PRINCIPAIS}

Este trabalho tem como objetivo principal, reformular a abordagem bayesiana dada por Bhattacharya et al (1995). Neste contexto, utilizamos algoritmos computacionais do tipo Gibbs Sampling/Metropolis-Hasting, para estimar os parâmetros de interesse, verificar o ajuste do modelo, e prever a sobrevivência de pacientes com doenças graves via distribuições preditivas. Mais um ponto importante que devemos destacar é a formulação de distribuições a priori informativas extraídas de médias condicionais a priori e a escolha das funções de ligações. 


\subsection{APRESENTAÇÃO DOS CAPÍTULOS}

Esta dissertação está organizada em oito capítulos e três apêndices. No capítulo 2 apresentamos o problema da análise de dados clínicos exponenciais levando-se em consideração alguns aspectos, do ponto de vista clássico. Apresentamos o modelo estatístico proposto inicialmente por Feigl e Zelen (1965) para a amostra completa, e uma aplicação a pacientes com leucemia, em seguida, numa outra formulação consideramos a presença de dados censurados. Também é assumido todos os pacientes entrando juntos, e entradas uniformes no estudo clínico. No final deste capítulo, temos uma generalização para várias variáveis auxiliares com dados completos e função de ligação linear, e logo após para dados censurados com função de ligação do tipo inversa proposta por Byar et al (1974), e uma aplicação relacionada ao câncer de próstata.

No capítulo 3 apresentamos uma inferência bayesiana exata sugerida por Bhattacharya et al (1995) para amostras com censuras tipo $\Pi$ e tipo III com o uso de uma única variável auxiliar influenciando a sobrevivência de pacientes num estudo clínico. Em seguida tem-se uma aplicação em dados de leucemia

Funções de ligações alternativas, analisadas sob o ponto de vista clássico e bayesiano são apresentadas no capítulo 4.

Formulamos no capítulo 5 uma inferência bayesiana para dados clínicos exponenciais com densidades a priori informativas com dados ampliados sob o contexto de modelos lineares generalizados.

Apresentamos no capítulo 6, dois métodos bayesianos para avaliar a adequação e selecionar modelos, respectivamente.

No capítulo 7, aplicamos o desenvolvimento teórico dos capítulos 4, 5 e 6 para analisar os dados de Feigl e Zelen (1965), e Zippin e Armitage (1966).

As conclusões finais e propostas futuras estão apresentadas no capítulo 8. 


\section{Capítulo 2}

\section{DADOS CLÍNICOS EXPONENCIAIS - ABORDAGEM CLÁSSICA}

Neste capítulo apresentaremos o problema da análise de dados clínicos exponenciais levando-se em consideração alguns aspectos da inferência clássica.

Desenvolvemos uma análise do modelo proposto para dados censurados e nãocensurados com uma e com várias variáveis auxiliares. Algumas ilustrações numéricas e gráficas também são apresentadas.

\subsection{MODELO ESTATÍSTICO SEM CENSURAS}

Descreveremos inicialmente o modelo estatístico para uma variável auxiliar.

Seja $t_{1}, t_{2}, \ldots, t_{n}$ uma amostra de $n$ tempos de sobrevivência independentes, onde o tempo de sobrevivência para o i-ésimo paciente tem a seguinte função de densidade de probabilidade:

$$
\left.f\left(t_{i} \mid \theta_{i}\right)=\theta_{i} \exp \left\{-\theta_{i} t_{i}\right\} I_{\left\{t_{i}\right.}>0\right\} \quad, \quad \theta_{i}>0
$$

onde,

$$
I_{\left\{t_{i}>0\right\}}= \begin{cases}1, & \text { se } t_{i}>0 \\ 0, & \text { caso contrário. }\end{cases}
$$

Além disso, seja $x_{1}, x_{2}, \ldots, x_{n}$ os valores observados de uma variável auxiliar $x$ tal que o valor esperado do tempo de sobrevivência para o i-ésimo paciente é: 


$$
E\left(t_{i}\right)=\frac{1}{\theta_{i}}=a+b x_{i}, \quad \text { (ligação linear). }
$$

Então, a função de verossimilhança dos n tempos de sobrevivência pode ser escrita como:

$$
\begin{array}{ll}
\ell(a, b)=\prod_{i=1}^{n} f\left(t_{i} \mid \theta_{i}\right)=\prod_{i=1}^{n} \theta_{i} \exp \left(-\theta_{i} t_{i}\right), & \text { portanto, } \\
\ell(a, b)=\left(\prod_{i=1}^{n}\left(a+b x_{i}\right)^{-1}\right) \cdot\left(\exp \left(-\sum_{i=1}^{n} t_{i}\left(a+b x_{i}\right)^{-1}\right)\right) . &
\end{array}
$$

Tomando o logaritmo de (2.3), temos que :

$$
\mathcal{L}(\mathrm{a}, \mathrm{b})=\ln \ell(\mathrm{a}, \mathrm{b})=-\sum_{\mathrm{i}=1}^{\mathrm{n}} \ln \left(\mathrm{a}+\mathrm{bx}_{\mathrm{i}}\right)-\sum_{\mathrm{i}=1}^{\mathrm{n}} \mathrm{t}_{\mathrm{i}}\left(\mathrm{a}+\mathrm{bx}_{\mathrm{i}}\right)^{-1} .
$$

Os estimadores de máxima verossimilhança podem ser encontrados de (2.4), resolvendo-se as seguintes equações através de métodos iterativos :

$$
\begin{aligned}
& \frac{\partial L}{\partial a}=0=-\sum_{i=1}^{n}\left(\hat{a}+\hat{b} x_{i}\right)^{-1}+\sum_{i=1}^{n} t_{i}\left(\hat{a}+\hat{b} x_{i}\right)^{-2}, \\
& \frac{\partial L}{\partial b}=0=-\sum_{i=1}^{n} x_{i}\left(\hat{a}+\hat{b} x_{i}\right)^{-1}+\sum_{i=1}^{n} x_{i} t_{i}\left(\hat{a}+\hat{b} x_{i}\right)^{-2} .
\end{aligned}
$$

Isto pode ser possível através do método de Newton-Raphson, (ver por exemplo, Lee 1992).

Se $\left(a_{k}, b_{k}\right)$ denotarem as estimativas na $k$-ésima iteração, os valores para a $(k+1)$ ésima iteração serão encontrados pela solução do seguinte sistema de equações :

$$
\left\{\begin{array}{l}
a_{k+1}=a_{k}-\left(B_{11}^{k} f_{1}^{k}+B_{12}^{k} f_{2}^{k}\right) \\
b_{k+1}=b_{k}-\left(B_{21}^{k} f_{1}^{k}+B_{22}^{k} f_{2}^{k}\right)
\end{array}\right.
$$

onde,

$$
f_{1}(a, b)=\frac{\partial L}{\partial a} \quad, \quad f_{2}(a, b)=\frac{\partial L}{\partial b} \quad
$$


$\mathrm{B}_{\mathrm{ij}}^{\mathrm{k}}$ é o ij-ésimo elemento de $\mathrm{J}^{-1}$ calculado em $\left(\mathrm{a}_{\mathrm{k}}, \mathrm{b}_{\mathrm{k}}\right)$,

$$
\begin{gathered}
J=\left[\begin{array}{ll}
\frac{\partial f_{1}}{\partial a} & \frac{\partial f_{1}}{\partial b} \\
\frac{\partial f_{2}}{\partial a} & \frac{\partial f_{2}}{\partial b}
\end{array}\right], \\
\frac{\partial f_{1}}{\partial a}=\sum_{i=1}^{n}\left(a_{k}+b_{k} x_{i}\right)^{-2}-2 \sum_{i=1}^{n} t_{i}\left(a_{k}+b_{k} x_{i}\right)^{-3} \\
\frac{\partial f_{1}}{\partial b}=\frac{\partial f_{2}}{\partial a}=\sum_{i=1}^{n} x_{i}\left(a_{k}+b_{k} x_{i}\right)^{-2}-2 \sum_{i=1}^{n} t_{i} x_{i}\left(a_{k}+b_{k} x_{i}\right)^{-3} \\
\frac{\partial f_{2}}{\partial b}=\sum_{i=1}^{n} x_{i}^{2}\left(a_{k}+b_{k} x_{i}\right)^{-2}-2 \sum_{i=1}^{n} t_{i} x_{i}^{2}\left(a_{k}+b_{k} x_{i}\right)^{-3}
\end{gathered}
$$

Uma estimativa inicial $\left(\mathrm{a}_{0}, \mathrm{~b}_{0}\right)$, sugerida por (2.2), pode ser obtida pelo ajuste de uma reta aos $n$ pares observados $\left(x_{i}, t_{i}\right)$.

Note que a solução $(\hat{a}, \hat{b})$ deverá estar no conjunto:

$$
\left\{(\mathrm{a}, \mathrm{b}): \mathrm{a}+\mathrm{bx}_{\mathrm{i}}>0, \forall \mathrm{x}_{\mathrm{i}}\right\}
$$

$O$ processo termina quando $f_{1}$ e $f_{2}$ estão próximas de zero, ou a diferença nos valores de a e b em duas iterações consecutivas é desprezível.

A matriz de variância-covariância assintótica para (â , $\hat{b})$, (ver Bickel e Doksum ,1977), será dada por:

$$
-\left[\begin{array}{cc}
E\left(\frac{\partial^{2} L}{\partial a^{2}}\right) & E\left(\frac{\partial^{2} L}{\partial a \partial b}\right) \\
E\left(\frac{\partial^{2} L}{\partial a \partial b}\right) & E\left(\frac{\partial^{2} L}{\partial b^{2}}\right)
\end{array}\right]^{-1}=\left[\begin{array}{cc}
\operatorname{var}(\hat{a}) & \operatorname{cov}(\hat{a}, \hat{b}) \\
\operatorname{cov}(\hat{a}, \hat{b}) & \operatorname{var}(\hat{b})
\end{array}\right] .
$$


Através de algumas manipulaçōes algébricas :

$$
\begin{aligned}
& \operatorname{var}(\hat{a})=\Delta^{-1} \sum_{i=1}^{n} x_{i}^{2}\left(a+b x_{i}\right)^{-2}, \\
& \operatorname{var}(\hat{b})=\Delta^{-1} \sum_{i=1}^{n}\left(a+b x_{i}\right)^{-2}, \\
& \operatorname{cov}(\hat{a}, \hat{b})=-\Delta^{-1} \sum_{i=1}^{n} x_{i}\left(a+b x_{i}\right)^{-2},
\end{aligned}
$$

onde:

$$
\Delta=\left[\sum_{i=1}^{n} x_{i}^{2}\left(a+b x_{i}\right)^{-2}\right]\left[\sum_{i=1}^{n}\left(a+b x_{i}\right)^{-2}\right]-\left[\sum_{i=1}^{n} x_{i}\left(a+b x_{i}\right)^{-2}\right]^{2}
$$

Para obter-se as estimativas de (2.8), os parâmetros $(\mathrm{a}, \mathrm{b})$ são substituídos por $(\hat{a}, \hat{b})$. Note que a probabilidade de sobrevivência para o i-ésimo paciente é :

$$
S_{i}(t)=\int_{t}^{\infty} f\left(\xi \mid \theta_{i}\right) d \xi=\exp \left(-\theta_{i} t\right)=\exp \left[-t\left(a+b x_{i}\right)^{-1}\right]
$$

Portanto, a estimativa para a função de sobrevivência pode ser obtida substituindo $(\mathrm{a}, \mathrm{b})$ por $(\hat{a}, \hat{b})$ em (2.9). Além disso fazendo uso da normalidade assintótica dos estimadores de máxima verossimilhança, pode-se obter um intervalo de confiança aproximado para o tempo médio de sobrevivência e para a função de sobrevivência. Seja :

$$
\mathrm{s}_{\mathrm{i}}^{2}=\operatorname{var}(\hat{\mathrm{a}})+\mathrm{x}_{\mathrm{i}}^{2} \operatorname{var}(\hat{\mathrm{b}})+2 \mathrm{x}_{\mathrm{i}} \operatorname{cov}(\hat{\mathrm{a}}, \hat{\mathrm{b}})
$$

Tem-se que :

$$
P\left\{\hat{a}+\hat{b} x_{i}-s z_{\alpha / 2} \leq a+b x_{i} \leq \hat{a}+\hat{b} x_{i}+s z_{\alpha / 2} l a, b, x_{i}\right\}=1-\alpha,
$$

onde $z_{\alpha / 2}$ é o $100(1-\alpha / 2)$ percentil superior da distribuição normal padrão. 


$$
\begin{aligned}
& \text { Fazendo-se : } c_{1 i}=\hat{a}+\hat{b} x_{i}-s z_{\alpha / 2}, \text { e } \quad c_{2 i}=\hat{a}+\hat{b} x_{i}+s z_{\alpha / 2}, \text { teremos, } \\
& P\left\{c_{1 i} \leq a+b x_{i} \leq c_{2 i} \mid a, b, x_{i}\right\}=1-\alpha, \quad \text { portanto, } \\
& P\left\{c_{1 i} \leq \theta^{-1} \leq c_{2 i} \mid a, b, x_{i}\right\}=P\left\{-c_{1 i}^{-1} t \leq-\theta_{i}^{-1} t \leq-c_{2 i}^{-1} t \mid a, b, x_{i}\right\}= \\
& =P\left\{\exp \left(-t c_{1}^{-1}\right) \leq S_{i}(t) \leq \exp \left(-t c_{2}^{-1}\right)\right\}=1-\alpha .
\end{aligned}
$$

A equação (2.11) define um intervalo de confiança bilateral aproximado para a função de sobrevivência do i-ésimo paciente.

A função de verossimilhança pode ser usada para obter contornos de igual verossimilhança em relação a $(\hat{a}, \hat{b})$. Um contorno $100 \alpha \%$ é um conjunto de pontos $(a, b)$ que satisfaz a seguinte propriedade :

$$
\begin{aligned}
& \ell(a, b)=\alpha \cdot \ell(\hat{a}, \hat{b}), \quad \text { ou } \\
& L(a, b)=\ln \alpha+L(\hat{a}, \hat{b})
\end{aligned}
$$

Um teste de adequação do modelo é fornecido comparando-se os números observados e esperados de pacientes mortos num certo intervalo de tempo. Seja $t_{i}(p)$ um valor tal que :

$$
\hat{S}_{i}\left[t_{i}(p)\right]=\exp \left[-t_{i}(p)\left(\hat{a}+\hat{b} x_{i}\right)^{-1}\right]=1-p
$$

Isto é, o paciente tendo variável auxiliar $x_{i}$ sobrevive além do tempo $t_{i}(p)$ com probabilidade 1 - p. Escolhendo-se $\mathrm{p}$ igual aos quantis probabilísticos por exemplo, $\mathrm{p}=0.25$; $0.50 ; 0.75$, teremos para o $\mathrm{i}$-ésimo paciente os quatro intervalos quartílicos $\left[0, \mathrm{t}_{\mathrm{i}}(0.25)\right]$; $\left[\mathrm{t}_{\mathrm{i}}(0.25), \mathrm{t}_{\mathrm{i}}(0.50)\right] ;\left[\mathrm{t}_{\mathrm{i}}(0.50), \mathrm{t}_{\mathrm{i}}(0.75)\right] ;\left[\mathrm{t}_{\mathrm{i}}(0.75), \infty\right]$. A probabilidade do i-ésimo paciente morrer em qualquer um destes intervalos é 0.25 . Tais intervalos podem ser construídos para cada paciente. Se o modelo está bem ajustado, podemos esperar que os tempos de sobrevivência 
estejam igualmente distribuídos nos quatro intervalos. Os números observados e esperados de pacientes podem ser comparados usando-se um teste de "bondade de ajuste".

\subsubsection{ILUSTRAÇÕES NUMÉRICAS E GRÁFICAS}

Os dados dispostos na Tabela 2.1 foram introduzidos por Feigl e Zelen (1965) para dois grupos de pacientes, os quais morrem de leucemia myelogenous aguda.. Os pacientes foram classificados dentro de dois grupos AG positivos e AG negativos de acordo com a presença ou ausência de uma característica morfológica de células brancas.

TABELA 2.1 - Tempo de sobrevivência observado e quantidade de glóbulos brancos

$$
\text { (AG positivo) } n=17
$$

(AG negativo) $n=16$

Quant. Glóbulos Brancos tempo de sobrevivência Quant. Glóbulos Brancos tempo de sobrevivência

\begin{tabular}{c|c|c|c} 
(QGB) & (semanas) & (QGB) & (semanas) \\
\hline 2300 & 65 & 4400 & 56 \\
\hline 750 & 156 & 3000 & 65 \\
\hline 4300 & 100 & 4000 & 17 \\
\hline 2600 & 134 & 1500 & 16 \\
\hline 6000 & 16 & 9000 & 22 \\
\hline 10500 & 108 & 5300 & 3 \\
\hline 10000 & 121 & 10000 & 4 \\
\hline 17000 & 4 & 19000 & 2 \\
\hline 5400 & 39 & 27000 & 3 \\
\hline 7000 & 143 & 28000 & 4 \\
\hline 9400 & 56 & 31000 & 3 \\
\hline 32000 & 26 & 26000 & 30 \\
\hline 35000 & 22 & 21000 & 4 \\
\hline 100000 & 1 & 79000 & 43 \\
\hline 100000 & 1 & 100000 & \\
\hline 52000 & 5 & 100000 & \\
\hline 100000 & 65 & & \\
\hline
\end{tabular}

Para a Tabela 2.1, a variável auxiliar $\mathbf{x}$ é o $\log _{10}$ da quantidade de glóbulos brancos (QGB) no instante do diagnóstico, conhecida como uma preditora do tempo de sobrevivência para esta doença (quanto mais alta a quantidade inicial de globulos brancos menor é a probabilidade de sobrevivência num específico intervalo de tempo). 
A função de verossimilhança para o primeiro grupo é mostrada na Figura 2.1. Ao seu lado, tem-se um mapa de contornos de igual verossimilhança. Note que o máximo fica perto da reta $a+5 b=0$, porque o tempo de sobrevivência previsto para altas quantidades de glóbulos brancos é muito baixo.

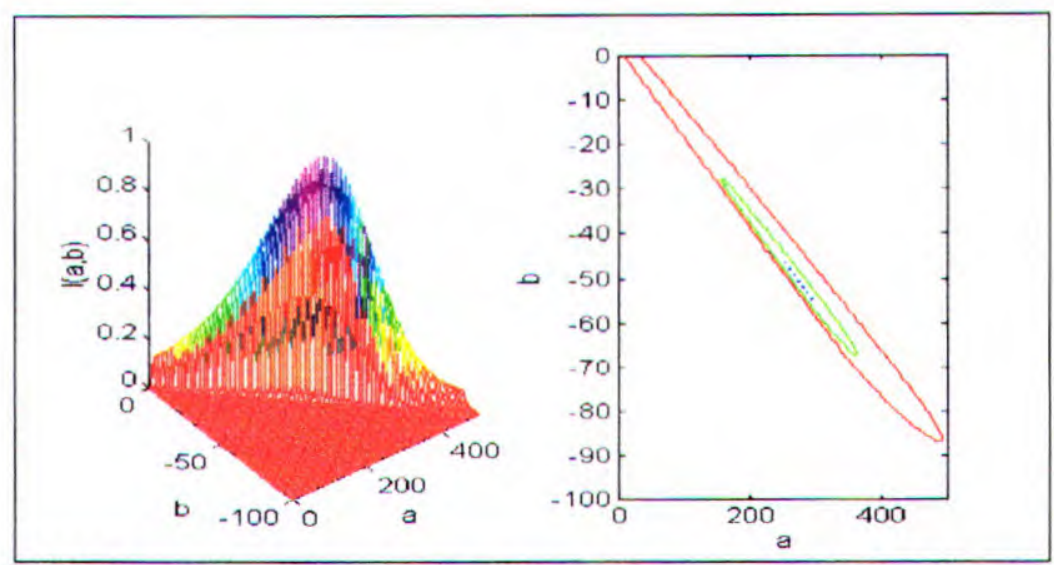

FIGURA 2.1 - Verossimilhança relativa e contornos de igual verossimilhança.

As estimativas de máxima verossimilhança $(\hat{a}, \hat{b})$ obtidas de $(2.5)$, com seus respectivos erros padrões e covariância segundo (2.8), para os dois grupos são apresentadas na Tabela 2.2 a seguir :

TABELA 2.2 - Estimativas de máxima verossimilhança, erros padrões e covariância para â e $\hat{b}$.

\begin{tabular}{c|c|c|c|c|c}
\hline Paciente do Grupo & $\hat{\mathrm{a}}$ & $\hat{\mathrm{b}}$ & $\sqrt{\operatorname{var}(\hat{\mathrm{a}})}$ & $\sqrt{\operatorname{var}(\hat{\mathrm{b}})}$ & $\operatorname{cov}(\hat{\mathrm{a}}, \hat{\mathrm{b}})$ \\
\hline AG positivo & 249 & -46 & 95.1 & 19.9 & -1888 \\
\hline AG negativo & 37 & -5 & 35.9 & 8.2 & -294 \\
\hline
\end{tabular}

Uma indicação da adequação do modelo é mostrada na Tabela 2.3, a qual dá os números esperados e observados de pacientes nos intervalos quartílicos. Os valores do qui-quadrado para a "bondade de ajustamento" , $\chi^{2}=\sum(\mathrm{O}-\mathrm{E})^{2} / \mathrm{E}$, têm dois graus de liberdade e são claramente não-significantes. 
TABELA 2.3 - Estatística $\chi^{2}$ para verificação do ajuste do modelo.

\begin{tabular}{|c|c|c|c|c|c|c|}
\hline \multirow{2}{*}{ Grupo } & \multirow{2}{*}{$\begin{array}{c}\text { Número } \\
\text { de } \\
\text { Pacientes }\end{array}$} & \multicolumn{4}{|c|}{ Intervalo Quartílico } & \multirow[b]{2}{*}{ Total } \\
\hline & & {$[0, t(.25)]$} & {$[t(.25), t(.50)]$} & {$[\mathrm{t}(.50), \mathrm{t}(.75)]$} & {$[\mathrm{t}(.75), \infty]$} & \\
\hline \multirow{3}{*}{ (AG Positivo) } & Observado & 5 & $\overline{4}$ & 3 & 5 & 17 \\
\hline & Esperado & 4.25 & 4.25 & 4.25 & 4.25 & 17 \\
\hline & & \multicolumn{5}{|c|}{$\chi^{2}=0.647(p=0.276)$} \\
\hline \multirow{3}{*}{ (AG Negativo) } & Observado & 4 & 3 & 2 & 7 & 16 \\
\hline & Esperado & 4 & 4 & 4 & 4 & 16 \\
\hline & & \multicolumn{5}{|c|}{$\chi^{2}=3.50(p=0.826)$} \\
\hline
\end{tabular}

Uma verificação gráfica da adequação do modelo é mostrada na Figura 2.2 . Aqui a distribuição de sobrevivência empírica é comparada com a curva de sobrevivência estimada associada a mediana da quantidade de glóbulos brancos para cada grupo. É notável que as probabilidades de sobrevivências para o grupo AG positivo dependam fortemente da quantidade de glóbulos brancos, ao passo que as distribuições de sobrevivência do grupo AG negativo possui somente uma fraca dependência da quantidade de glóbulos brancos. Isto está refletido no fato da diferença nos valores $\hat{b}$ ( -46 vs. -5 ). Quanto maior o l $\hat{b}$ ।, maior a dependência da quantidade de glóbulos brancos. A melhoria geral da sobrevivência dos pacientes AG positivos está relacionada ao fato de que para alguma quantidade de glóbulos brancos especificada, a curva de sobrevivência do grupo AG positivo encontra-se acima da curva de sobrevivência do grupo AG negativo.

(a)

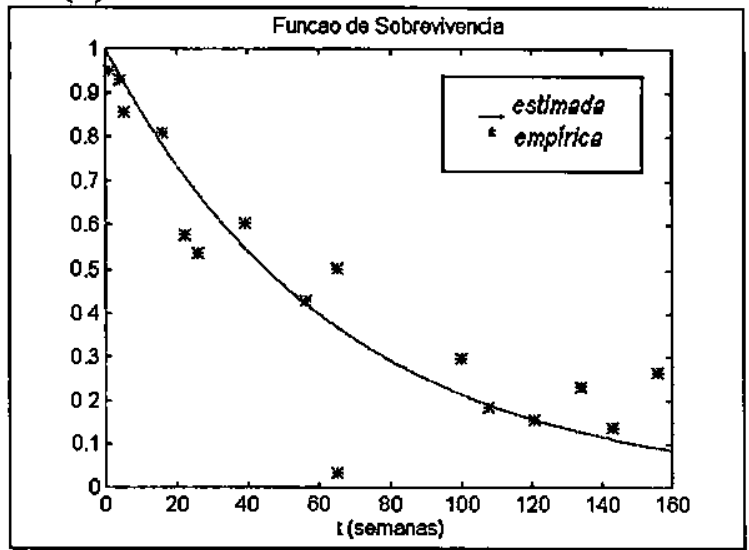

(b)

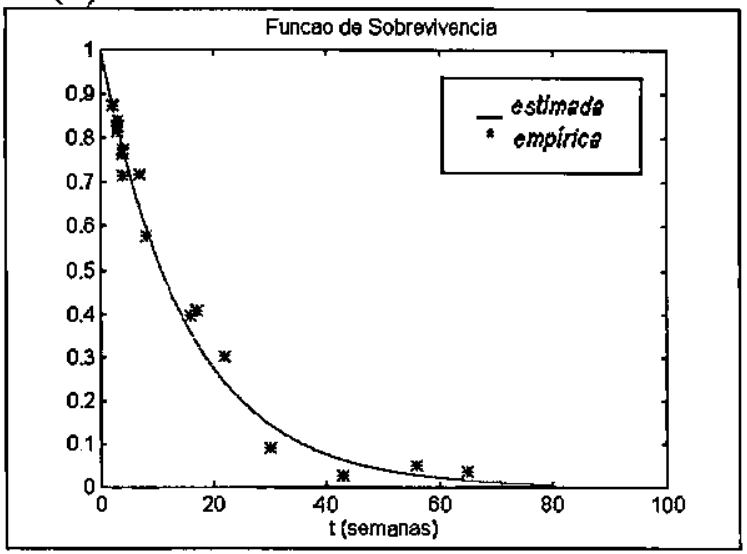

FIGURA 2.2 - Função de sobrevivência para os grupos, AG - positivo em (a) e AG - negativo em (b). 
Um outro modo de comparar os dois grupos é fazer um teste de significância aproximado para a diferença dos tempos médios de sobrevivência, i.e., $\left(a_{1}+b_{1} x\right)-\left(a_{2}+b_{2} x\right)$. Note que a comparação depende de um valor fixo da variável auxiliar $\mathbf{x}$. Portanto, uma aproximação normal para os desvios (sob a hipótese de que os dois grupos possuem os mesmos parâmetros) é :

$$
\mathrm{z}=\Delta / \mathrm{s}_{\Delta}
$$

Então, rejeitamos $\quad H_{0}:\left(a_{1}+b_{1} x\right)-\left(a_{2}+b_{2} x\right)=0 \quad$ se :

$$
\left|z=\Delta / s_{\Delta}\right| \geq z_{\alpha / 2}
$$

onde,

$$
\begin{gathered}
\Delta=\left(\hat{a}_{1}-\hat{a}_{2}\right)+x\left(\hat{b}_{1}-\hat{b}_{2}\right), \\
s_{\Delta}^{2}=\operatorname{var}\left(\hat{a}_{1}\right)+\operatorname{var}\left(\hat{a}_{2}\right)+2 x\left[\operatorname{cov}\left(\hat{a}_{1}, \hat{b}_{1}\right)+\operatorname{cov}\left(\hat{a}_{2}, \hat{b}_{2}\right)\right]+x^{2}\left[\operatorname{var}\left(\hat{b}_{1}\right)+\operatorname{var}\left(\hat{b}_{2}\right)\right],
\end{gathered}
$$

e $\quad z_{\alpha / 2}$ é o $100(1-\alpha / 2)$ percentil superior da distribuição normal-padrão.

Uma escolha apropriada de um valor fixo da variável auxiliar $\mathbf{x}$ é mediana para os dois grupos tomados juntos, i.e., 10500. Substituindo em (2.14) $\operatorname{com} x=\log _{10}(10500)=4.02$, teremos:

$$
\frac{\Delta}{S_{\Delta}}=\frac{46.46}{17.34}=2.68
$$

o qual é significante ao nível 0.008. Portanto, os tempos médios de sobrevivências são diferentes. 


\subsection{MODELO ESTATÍSTICO COM CENSURAS}

Nesta seção generalizaremos o modelo de Feigl e Zelen (1965) para mostrar como a estimação dos parâmetros do modelo de regressão linear pode ser feita, quando alguns pacientes ainda estão vivos após completar-se o estudo. Para estudos de diferentes durações, erros padrões assintóticos das estimativas são obtidos para um conjunto fixo de valores da variável auxiliar (logaritmo da quantidade de glóbulos brancos). Primeiro todos os pacientes entram juntos no estudo, e em seguida, assume-se intervalos de tempo uniformes entre entradas sucessivas de pacientes.

Novamente assumiremos como válidas as equações (2.1) e (2.2), onde n pacientes entram no estudo, d destes morrem e $\mathrm{s}=\mathrm{n}-\mathrm{d}$ ainda estão vivos no fim do estudo. Para o j-ésimo paciente cuja morte ocorre no tempo $t_{j}$, o valor da variável auxiliar é $x_{j}$; para o k-ésimo paciente ainda vivo, tendo tempo de sobrevivência $t_{k}$, a variável auxiliar possui valor $x_{k}$.

A função de verossimilhança para o conjunto de n observações é então :

$$
\ell(a, b)=\prod_{j=1}^{d} \theta_{j} \exp \left(-\theta_{j} t_{j}\right) \prod_{k=d}^{s} \exp \left(-\theta_{k} t_{k}\right)
$$

A log-verossimilhança é:

$$
\begin{aligned}
L & =\ln \ell(a, b)=\sum_{j=1}^{d} \ln \theta_{j}-\sum_{j=1}^{d} \theta_{j} t_{j}-\sum_{k=1}^{s} \theta_{k} t_{k} \\
& =\sum_{j=1}^{d} \ln \left(a+b x_{j}\right)^{-1}-\sum_{j=1}^{d}\left(a+b x_{j}\right)^{-1} t_{j}-\sum_{k=1}^{s}\left(a+b x_{k}\right)^{-1} t_{k} .
\end{aligned}
$$

Podemos obter através de métodos iterativos os valores â e $\hat{b}$ que maximizam a verossimilhança da amostra de observações igualando a zero as primeiras derivadas parciais de (2.16), estas são: 


$$
\begin{aligned}
& \frac{\partial \mathrm{L}}{\partial \mathrm{a}}=-\sum_{j} \theta_{j}+\sum_{j} \mathrm{t}_{\mathrm{j}} \theta_{\mathrm{j}}^{2}+\sum_{\mathrm{k}} \mathrm{t}_{\mathrm{k}} \theta_{\mathrm{k}}^{2}, \\
& \frac{\partial \mathrm{L}}{\partial \mathrm{b}}=-\sum_{\mathrm{j}} \mathrm{x}_{\mathrm{j}} \theta_{\mathrm{j}}+\sum_{\mathrm{j}} \mathrm{t}_{\mathrm{j}} \mathrm{x}_{\mathrm{j}} \theta_{\mathrm{j}}^{2}+\sum_{\mathrm{k}} \mathrm{t}_{\mathrm{k}} \mathrm{x}_{\mathrm{k}} \theta_{\mathrm{k}}^{2} .
\end{aligned}
$$

As segundas derivadas parciais de $\mathrm{L}$ são:

$$
\begin{aligned}
& A=\frac{\partial^{2} L}{\partial a^{2}}=\sum_{j} \theta_{j}^{2}-2 \sum_{j} t_{j} \theta_{j}^{3}-2 \sum_{k} t_{k} \theta_{k}^{3}, \\
& B=\frac{\partial^{2} L}{\partial b^{2}}=\sum_{j} x_{j}^{2} \theta_{j}^{2}-2 \sum_{j} t_{j} x_{j}^{2} \theta_{j}^{3}-2 \sum_{k} t_{k} x_{k}^{2} \theta_{k}^{3}, \\
& C=\frac{\partial^{2} L}{\partial a \partial b}=\sum_{j} x_{j} \theta_{j}^{2}-2 \sum_{j} t_{j} x_{j} \theta_{j}^{3}-2 \sum_{k} t_{k} x_{k} \theta_{k}^{3} .
\end{aligned}
$$

As fórmulas para a estimação assintótica de â e $\hat{b}$ são :

$$
\operatorname{Var}(\hat{\mathrm{a}})=-\mathrm{B} /\left(\mathrm{AB}-\mathrm{C}^{2}\right)
$$

e

$$
\operatorname{Var}(\hat{\mathrm{b}})=-\mathrm{A} /\left(\mathrm{AB}-\mathrm{C}^{2}\right)
$$

onde $(\hat{a}+\hat{b} x)^{-1}$ é substituído por $\theta$ em $(2.17)-(2.19)$.

A seguir, iremos retornar as aplicações da subseção 2.1.1. A Tabela 2.4 mostra a quantidade de glóbulos brancos, o tempo de acompanhamento e o estado de cada paciente. Cinco destes pacientes foram selecionados aleatoriamente para serem sobreviventes até o fim do estudo. Portanto, tem-se agora a presença de censuras. 
TABELA 2.4 - Quantidade de Glóbulos Brancos (QGB), período de acompanhamento e o estado do paciente com leucemia (Zippin e Armitage,1966).

\begin{tabular}{c|c|c|c}
\hline $\begin{array}{c}\text { QGB } \\
(\mathbf{1 0 0 0})\end{array}$ & $\begin{array}{c}\log _{10} \mathbf{Q G B} \\
(\mathbf{x})\end{array}$ & $\begin{array}{c}\text { Período de } \\
\text { Acompanhamento (t) } \\
\text { (semanas) }\end{array}$ & $\begin{array}{c}\text { Estado no fim do } \\
\text { Acompanhamento } \\
\text { vivo (A) ou morto (D) }\end{array}$ \\
\hline 0.75 & 2.8751 & 156 & $\mathrm{D}$ \\
\hline 2.3 & 3.3617 & 65 & $\mathrm{D}$ \\
\hline 2.6 & 3.4150 & $\mathbf{1 3}$ & $\mathrm{A}$ \\
\hline 4.3 & 3.6335 & 100 & $\mathrm{D}$ \\
\hline 5.4 & 3.7324 & 39 & $\mathrm{D}$ \\
\hline 6.0 & 3.7782 & 16 & $\mathrm{D}$ \\
\hline 7.0 & 3.8451 & 143 & $\mathbf{A}$ \\
\hline $\mathbf{9 . 4}$ & $\mathbf{3 . 9 7 3 1}$ & $\mathbf{3 4}$ & $\mathrm{D}$ \\
\hline 10.0 & 4.0000 & 121 & $\mathrm{D}$ \\
\hline 10.5 & 4.0212 & 108 & $\mathrm{D}$ \\
\hline 17.0 & 4.2304 & 4 & $\mathbf{A}$ \\
\hline $\mathbf{3 2 . 0}$ & $\mathbf{4 . 5 0 5 2}$ & $\mathbf{5}$ & $\mathrm{D}$ \\
\hline 35.0 & 4.5441 & $\mathbf{1 3}$ & $\mathbf{A}$ \\
\hline 52.0 & 4.7160 & 5 & $\mathrm{D}$ \\
\hline $\mathbf{1 0 0 . 0}$ & $\mathbf{5 . 0 0 0 0}$ & 1 & $\mathrm{D}$ \\
\hline 100.0 & 5.0000 & 1 & \\
\hline 100.0 & 5.0000 & 65 & \\
\hline & & & $\mathrm{D}$ \\
\hline
\end{tabular}

Para estes dados, através do método de Newton - Raphson, obtivemos $\hat{a}=257$ e $\hat{b}=$ - 46, valores para os quais a função de verossimilhança é máxima e empregando as equações (2.20) e (2.21) e tomando as raízes quadradas, encontramos os erros padrões (SE): $\mathrm{SE}(\hat{a})=$ 121.9 e $\operatorname{SE}(\hat{b})=26.0$. Feigl e Zelen (1965) obtiveram os valores $\hat{a}=240$ e $\hat{b}=-44$, $\operatorname{SE}(\hat{a})=95.5$ e $\operatorname{SE}(\hat{b})=20.1$ baseando-se nas informações completas de sobrevivência .

Percorrendo a superfície de resposta, Zippin e Armitage (1966) obtiveram os valores : $\hat{a}=240$ e $\hat{b}=-42 ; \operatorname{SE}(\hat{a})=104.2$ e $\operatorname{SE}(\hat{b})=23.5$. A Tabela 2.5 a abaixo, apresenta um resumo destas estimativas.

TABELA 2.5 - Estimativas de máxima verossimilhança e, erros padrões para â e $\hat{b}$.

\begin{tabular}{c|c|c|c}
\hline & $\begin{array}{c}\text { Método de Newton- } \\
\text { Raphson }\end{array}$ & $\begin{array}{c}\text { Feigl e Zelen } \\
\mathbf{( 1 9 6 5 )}\end{array}$ & $\begin{array}{c}\text { Zippin e Armitage } \\
\mathbf{( 1 9 6 6 )}\end{array}$ \\
\hline$\hat{\mathrm{a}}$ & 257 & 240 & 240 \\
\hline$\hat{\mathrm{b}}$ & -46 & -44 & -42 \\
\hline $\mathrm{SE}(\hat{\mathrm{a}})$ & 121.9 & 95.5 & 104.2 \\
\hline $\mathrm{SE}(\hat{\mathrm{b}})$ & 26.0 & 20.1 & 23.5 \\
\hline
\end{tabular}




\subsubsection{VARIÂNCIAS ASSINTÓTICAS DAS ESTIMATIVAS DE a e b}

Suponha que o i-ésimo indivíduo entra no estudo antes do fim do tempo de acompanhamento $T_{i}$ e para d mortes o período de acompanhamento observado $t_{j}$ é menor que $T_{j}$. Para $s=n-d$ sobreviventes o período de observação é $t_{k}=T_{k}$.

Para o i-ésimo indivíduo, a probabilidade de morte antes de $\mathrm{T}_{\mathrm{i}}$ é $\left(1-\mathrm{e}^{-\theta_{\mathrm{i}} \mathrm{T}_{\mathrm{i}}}\right)$ e sua contribuição para os valores esperados das segundas derivadas parciais de Lé dada por :

$$
E_{i}(\alpha)=E_{t_{i}}\left[\theta_{i}^{2} x_{i}^{\alpha} I_{\left[t_{i}<T_{i}\right]}-2 t_{i} x_{i}^{\alpha} \theta_{i}^{3} I_{\left[t_{i}<T_{i}\right]}-2 t_{i} x_{i}^{\alpha} \theta_{i}^{3} I_{\left[t_{i}=T_{i}\right]}\right],
$$

onde,

$$
\begin{aligned}
& \mathrm{I}_{\left[\mathrm{t}_{\mathrm{i}}<\mathrm{T}_{\mathrm{i}}\right]}= \begin{cases}1, & \text { se } \mathrm{t}_{\mathrm{i}}<\mathrm{Ti} \\
0, & \text { caso contrário } .\end{cases} \\
& \mathrm{I}_{\left[\mathrm{t}_{\mathrm{i}}=\mathrm{T}_{\mathrm{i}}\right]}= \begin{cases}1, & \text { se } \mathrm{t}_{\mathrm{i}}=\mathrm{Ti} \\
0, & \text { caso contrário } .\end{cases}
\end{aligned}
$$

Portanto:

$$
\begin{aligned}
& E_{i}(\alpha)=\theta_{i}^{2} x_{i}^{\alpha} \cdot P\left[t_{i}<T_{i}\right]-2 x_{i}^{\alpha} \theta_{i}^{3} \cdot E_{t_{i}}\left[t_{i} I_{\left[t_{i}<T_{i}\right]}\right]-2 x_{i}^{\alpha} \theta_{i}^{3} \cdot E_{t_{i}}\left[t_{i} I_{\left[t_{i}=T_{i}\right]}\right] \\
& E_{i}(\alpha)=\left(1-e^{-\theta_{i} T_{i}}\right) \theta_{i}^{2} x_{i}^{\alpha}-2 x_{i}^{\alpha} \theta_{i}^{3} \int_{0}^{T_{i}} t_{i} \theta_{i} e^{-\theta_{i} t_{i}} d t_{i}-2 x_{i}^{\alpha} \theta_{i}^{3} T_{i} \cdot P\left[t_{i}=T_{i}\right] \\
& E_{i}(\alpha)=\left(1-e^{-\theta_{i} T_{i}}\right) \theta_{i}^{2} x_{i}^{\alpha}-2 x_{i}^{\alpha} \theta_{i}^{3} \int_{0}^{T_{i}} t_{i} \theta_{i} e^{-\theta_{i} t_{i}} d t_{i}-2 x_{i}^{\alpha} \theta_{i}^{3} T_{i} e^{-\theta_{i} T_{i}},
\end{aligned}
$$

onde,

$$
\alpha= \begin{cases}0, & \text { para } \partial^{2} \mathrm{~L} / \partial \mathrm{a}^{2} \\ 1, & \text { para } \partial^{2} \mathrm{~L} / \partial \mathrm{a} \partial \mathrm{b} \\ 2, & \text { para } \partial^{2} \mathrm{~L} / \partial \mathrm{b}^{2}\end{cases}
$$

Desde que 


$$
\int_{0}^{T_{i}} t_{i} \theta_{i} e^{-\theta_{i} t_{i}} d t_{i}=\frac{1}{\theta_{i}^{2}}\left[\left(1-e^{-\theta_{i} T_{i}}\right)-\theta_{i} T_{i} e^{-\theta_{i} T_{i}}\right],
$$

a equação (2.22) se reduz a

$$
E_{i}(\alpha)=-x_{i}^{\alpha} \theta_{i}^{2}\left(1-e^{-\theta_{i} T_{i}}\right)
$$

\subsubsection{TODOS OS PACIENTES ENTRAM JUNTOS NO ESTUDO}

Assumiremos que todos os pacientes entram juntos no tempo $\mathrm{T}$ antes de encerrar $\mathrm{o}$ acompanhamento, i.e., $\mathrm{T}_{\mathrm{i}}=\mathrm{T}$ para todos os pacientes. Admitiremos uma distribuição fixa dos x's como dado na seção 2.1 , e que as estimativas 249 e - 46 são os verdadeiros valores dos parâmetros a e b . Empregando os valores esperados das segundas dertivadas de L como é dado em (2.23), os erros padrões assintóticos das estimativas de máxima verossimilhança de a e b estão dados na Tabela 2.6 a seguir.

TABELA 2.6 - Erros padrões assintóticos das estimativas de máxima verossimilhança, para $\mathrm{a}=249 \mathrm{e} \mathrm{b}=-46, \mathrm{e}$ assumindo que todos os pacientes entram juntos no estudo.

\begin{tabular}{c|c|c}
\hline $\begin{array}{c}\text { Duração do estudo } \\
\text { semanas (T) }\end{array}$ & SE $(\hat{\mathbf{a}})$ & SE $(\hat{\mathrm{b}})$ \\
\hline 25 & 170 & 35 \\
\hline 50 & 133 & 27 \\
\hline 100 & 111 & 23 \\
\hline 200 & 100 & 21 \\
\hline$\infty$ & 96 & 20 \\
\hline
\end{tabular}

Note que, para um aumento no tempo de estudo os erros padrões reduzem-se e tendem aos os mesmos valores, iguais aqueles obtidos para os dados não-censurados na seção 2.1.1. 


\subsubsection{ENTRADA UNIFORME NO ESTUDO}

Vamos a seguir, assumir que $T_{i}$ tem uma distribuição retangular em $(0, T)$, correspondendo a pacientes entrando no estudo a uma taxa uniforme, i.e., em intervalos regulares entre o tempo zero e o tempo T. Sob esta suposição, podemos denotar os valores de $\mathrm{E}_{\mathrm{i}}(\alpha)$ por $\mathrm{E}_{\mathrm{i}}^{*}(\alpha)$. Então :

$$
\begin{aligned}
\mathrm{E}_{\mathrm{i}}^{*}(\alpha) & =-\mathrm{x}_{\mathrm{i}}^{\alpha} \theta_{\mathrm{i}}^{2}\left\{1-\frac{1}{\mathrm{~T}} \int_{0}^{\mathrm{T}} \mathrm{e}^{-\theta_{\mathrm{i}} \mathrm{T}_{\mathrm{i}}} \mathrm{dt} \mathrm{t}_{\mathrm{i}}\right\} \\
& =-\mathrm{x}_{\mathrm{i}}^{\alpha} \theta_{\mathrm{i}}^{2}\left\{1-\frac{1}{\theta_{\mathrm{i}} \mathrm{T}}\left(1-\mathrm{e}^{-\theta_{\mathrm{i}} \mathrm{T}}\right)\right\} .
\end{aligned}
$$

Novamente, utilizando as 17 observações de Feigl e Zelen (1965) e assumindo verdadeiro os valores $\mathrm{a}=249 \mathrm{e} \mathrm{b}=-46$, obtém-se os valores mostrados na Tabela 2.7 para os erros padrões assintóticos de â e $\hat{b}$.

TABELA 2.7 - Erros padrões assintóticos das estimativas de máxima verossimilhança, para $\mathrm{a}=249$ e $\mathrm{b}=-46 \mathrm{e}$ assumindo entrada de pacientes uniformemente no estudo de duração $\mathrm{T}$.

\begin{tabular}{c|c|c}
\hline $\begin{array}{c}\text { Duração do estudo } \\
\text { semanas (T) }\end{array}$ & SE $(\hat{\mathrm{a}})$ & SE $(\hat{\mathrm{b}})$ \\
\hline 25 & 232 & 48 \\
\hline 50 & 176 & 36 \\
\hline 100 & 139 & 29 \\
\hline 200 & 118 & 24 \\
\hline$\infty$ & 96 & 20 \\
\hline
\end{tabular}

Uma comparação das Tabelas 2.6 e 2.7 mostra , que a precisão das estimativas baseadas em entradas uniformes é aproximadamente a mesma correspondendo ao estudo com metade da duração no qual todos os pacientes entram juntos.

Aqui, como na Tabela 2.6, os erros padrões baseados num estudo de 200 semanas de duração são aproximadamente metade daqueles de 25 semanas de estudo. 


\subsection{MODELOS ESTATÍSTICOS RELACIONADOS A VÁRIAS VARIÁVEIS AUXILIARES}

\subsubsection{MODELO ESTATÍSTICO SEM CENSURAS}

A generalização dos procedimentos apresentados quando o experimento clínico possui $\mathrm{k}$ tratamentos é direta. Seja o i-ésimo paciente recebendo tratamento $j$ e tendo tempo de sobrevivência $t_{i j}$ para $i=1,2, \ldots, n_{j} ; j=1,2, \ldots, k$ ( $n_{j}$ pacientes recebem tratamento $j$ ). Assumindo o modelo (2.1) para os tempos de sobrevivência, com :

$$
E\left(t_{i j}\right)=a_{j}+b x_{i j}
$$

onde $\mathrm{x}_{\mathrm{ij}}$ é a variável auxiliar (Feigl e Zelen, 1965), a log-verossimilhança pode ser escrita como:

$$
L\left(a_{1}, a_{2}, \ldots, a_{k}, b\right)=\sum_{j=1}^{k} L\left(a_{j}, b\right),
$$

onde,

$$
L\left(a_{j}, b\right)=-\sum_{i=1}^{n_{j}} \ln \left(a_{j}+b x_{i j}\right)-\sum_{i=1}^{n_{j}} t_{i j}\left(a_{j}+b x_{i j}\right)^{-1} .
$$

As equações cujas soluções resultam nos estimadores de máxima verossimilhança são:

$$
\begin{aligned}
& \sum_{i=1}^{n_{j}}\left(\hat{a}_{j}+\hat{b} x_{i j}\right)^{-1}=\sum_{i=1}^{n_{j}} t_{i j}\left(\hat{a}_{j}+\hat{b} x_{i j}\right)^{-2}, j=1,2, \ldots, k ; \\
& \sum_{j=1}^{k} \sum_{i=1}^{n_{j}} x_{i j}\left(\hat{a}_{j}+\hat{b} x_{i j}\right)^{-1}=\sum_{j=1}^{k} \sum_{i=1}^{n_{j}} t_{i j} x_{i j}\left(\hat{a}_{j}+\hat{b} x_{i j}\right)^{-2} .
\end{aligned}
$$

Estas são de difícil solução, e um método iterativo se faz necessário. 


\subsubsection{MODELO ESTATÍSTICO COM CENSURAS}

O modelo apresentado na seção 2.2 será estendido para determinar a importância relativa da presença de vários sintomas em relação ao tempo de sobrevivência sobrevivência.

Suponha que o i-ésimo indivíduo em estudo possui os seguintes valores de $\mathrm{k}$ variáveis auxiliares $\mathrm{X}_{\mathrm{i} 0}, \mathrm{X}_{\mathrm{i} 1}, \ldots, \mathrm{X}_{\mathrm{ik}}$. A variável $\mathrm{X}_{\mathrm{i} 0} \in$ "dummy" e identicamente igual a 1. Para as outras variáveis, $X_{i j}=1$, se o paciente $i$ tem a característica $\mathrm{j}$ e $X_{i j}=0$, caso contrário, $j=1,2$, $\ldots, k$. Além disso, suponha que os correspondentes tempos de sobrevivência $t_{1}, t_{2}, \ldots, t_{n}$ estejam cada um relacionado ao risco individual $\theta_{i}$, o qual é dado como uma combinação linear das variáveis auxiliares :

$$
\theta_{i}=b_{0} x_{i 0}+b_{1} x_{i 1}+b_{2} x_{i 2}+\cdots+b_{k} x_{i k}=\sum_{j=0}^{k} b_{j} x_{i j}, \quad i=1, \ldots, n .
$$

O termo $b_{0}$ representa o risco básico (intercepto), isto é, $b_{0}$ representa a taxa de mortalidade $\theta_{i}$, sob a condição que todos os $X_{i j}$ são iguais a zero.

A função de verossimilhança para uma amostra censurada é, obtida de forma análoga, através de (2.15), isto é :

$$
\ell=\prod_{i=1}^{d} \theta_{i} \exp \left(-\theta_{i} t_{i}\right) \prod_{j=d+1}^{n} \exp \left(-\theta_{j} t_{j}\right)
$$

Aqui estamos supondo $\mathrm{d}$ mortes e $\mathrm{s}=\mathrm{n}$ - $\mathrm{d}$ sobreviventes. A equação (2.26) pode ser reescrita, introduzindo-se uma variável $z_{i}$ para denotar se a i-ésima observação é censurada, logo

$$
\ell=\prod_{\mathrm{i}=1}^{\mathrm{n}} \exp \left(\mathrm{z}_{\mathrm{i}} \ln \theta_{\mathrm{i}}-\theta_{\mathrm{i}} \mathrm{t}_{\mathrm{i}}\right)
$$

onde, 


$$
z_{i}=\left\{\begin{array}{l}
1, \text { se o paciente } i \text { morre no tempo } t_{i}, \\
0, \text { se paciente } i \text { está vivo no tempo } t_{i}
\end{array} .\right.
$$

Substituindo $\theta_{\mathrm{i}}$ dado pela equação (2.25), a log-verossimilhança será dada por:

$$
L=\ln \ell=\sum_{i=1}^{n}\left[z_{i} \ln \left(\sum_{j=0}^{k} b_{j} x_{i j}\right)-\left(\sum_{j=0}^{k} b_{j} x_{i j}\right) t_{i}\right]
$$

As $k+1$ equações não-lineares cujas soluções fornecem as estimativas de máxima verossimilhança são dadas por :

$$
\frac{\partial L}{\partial b_{j}}=\sum_{i=1}^{n}\left(z_{i} X_{i j} / \sum_{j=0}^{k} b_{j} X_{i j}\right)-\sum_{i=1}^{n} X_{i j} t_{i}=0 .
$$

O modelo apresentado acima com taxa de risco (2.25), foi usado por Byar et al. (1974) para um estudo baseado em 11 variáveis auxiliares e dados censurados de 1824 pacientes com um avançado câncer de próstata .

As estimativas dos coeficientes de regressão para as 11 variáveis e seus desvios padrões são dados na Tabela 2.8 a seguir . Uma idéia da importância relativa das variáveis é ilustrada pela magnitude dos coeficientes de regressão. Por exemplo, a variável 8 (totalmente enfermo) aparece como a mais importante. 
TABELA 2.8 - Estimativas dos coeficientes de regressão e seus desvios padrões.

\begin{tabular}{|c|c|c|c|c|}
\hline$N^{\circ}$ & Variável & $\hat{b}_{j}^{*}$ & SD & $\hat{b}_{j} / S D$ \\
\hline 0 & Taxa de mortalidade & 7.88 & 0.64 & 12.31 \\
\hline 1 & Dor devido ao câncer & -0.20 & 1.75 & -0.11 \\
\hline 2 & Acido fosfático $^{+} \quad 1.1-2.0$ & 4.15 & 1.40 & 2.96 \\
\hline 3 & Acido fosfático $^{+} \quad 2.1-5.0$ & 6.94 & 1.93 & 3.60 \\
\hline 4 & Acido fosfático $^{+}>5$ & 11.04 & 2.09 & 5.28 \\
\hline 5 & Dilatação da uretra & 4.68 & 2.31 & 2.03 \\
\hline$\overline{6}$ & 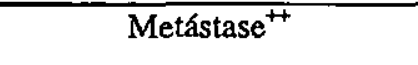 & 7.36 & 1.91 & 3.85 \\
\hline 7 & Parcialmente enfermo & 11.18 & 2.39 & 4.68 \\
\hline 8 & Totalmente enfermo & 21.55 & 8.48 & 2.54 \\
\hline 9 & Peso $<130$ libras & 3.50 & 1.34 & 2.61 \\
\hline 10 & Hemoglobina $<12 \mathrm{~g} / 100 \mathrm{mI}$ & 5.44 & 1.49 & 3.65 \\
\hline 11 & Idade $\geq 70$ anos & 3.02 & 0.85 & 3.55 \\
\hline
\end{tabular}

Note que o prognóstico para um paciente parcialmente enfermo é melhor que para um paciente totalmente enfermo. Os desvios padrões das estimativas foram grandemente afetados pelo número de pacientes no qual a variável esteve presente. Um pequeno desvio está associado a variável 0 (taxa básica de mortalidade), pois todos os pacientes contribuíram para sua estimação, mas a variável 8 (totalmente enfermo), embora tenha estimativa para o coeficiente de regressão grande, possui também um grande desvio padrão, visto que muito poucos pacientes estavam totalmente enfermos no começo do estudo.

Uma previsão da taxa de mortalidade poderá ser obtida para cada paciente pela simples adição dos coeficientes de regressão correspondentes à característica presente no diagnóstico através da expressão (2.25). 


\section{Capítulo 3}

\section{DADOS CLÍNICOS EXPONENCIAIS - ABORDAGEM BAYESIANA EXATA}

Neste capítulo apresentamos uma inferência bayesiana exata para dados de sobrevivência, surgindo de um modelo de tempos de sobrevivência exponencial, para amostras com censuras tipo II e tipo III com o uso de uma única variável auxiliar $\mathbf{x}$ influenciando a sobrevivência de pacientes num estudo clínico. Em seguida, tem-se uma aplicação em dados de leucemia.

\subsection{MODELO ESTATÍSTICO COM CENSURAS DO TIPO III}

Assumiremos o modelo de sobrevivência exponencial com função densidade de probabilidade (fdp) dada pela equação (2.1) e que $\mathbf{n}$ pacientes entram num estudo clínico ao mesmo tempo e são acompanhados até $\mathbf{d}$ mortes, $(0<\mathrm{d} \cdot \mathrm{n})$. Os tempos até a morte registrados são denotados por $t_{1}<t_{2}<\cdots<t_{d}$. Portanto, temos a censura do tipo $\Pi$, de modo que os $t_{i}$ são desconhecidos para $\mathrm{i} \geq \mathrm{d}+1$.

Denotaremos por $\mathbf{x}_{1}$ o valor de uma variável auxiliar $\mathbf{x}$ correspondendo ao i-ésimo paciente $(i=1,2, \ldots, n)$. Por exemplo no estudo da leucemia, a quantidade de glóbulos brancos (QGB) e o tempo de diagnóstico são variáveis úteis para a análise de dados de sobrevivência. Portanto, os dados consistem no vetor de observações $\left(x_{i}, t_{i}\right), i=1,2, \ldots, n$, onde $t_{i}>t_{d}$ para $i=d+1, d+2, \ldots, n$. Este conjunto de dados será representado por $D_{l}$. 
Se o valor da variável auxiliar x para o i-ésimo paciente é $x_{i}(i=1,2, \ldots, n)$, a taxa de risco $\theta_{\mathrm{i}}$ para este paciente é uma função de $x_{i}$. Sob a hipótese de que altos valores da variável auxiliar x correspondem ao aumento da gravidade da doença em estudo, claramente podemos supor:

$$
\theta=\beta x, \quad(\beta>0) \quad \text { (ligação inversa) }
$$

isto é,

$$
E\left(t_{i}\right)=(\beta x)^{-1} \text {. }
$$

Portanto, através da fdp (2.1), a função densidade de probabilidade para o tempo sobrevivência de um paciente com uma variável auxiliar $\mathrm{x}$, será dada pela expressão:

$$
f(t \mid \beta)=\beta x e^{-\beta x t}, \quad(0 \leq t<\infty, \quad \beta>0, \quad x>0)
$$

\subsection{DENSIDADES A PRIORI}

Denotaremos por $\pi(\beta)$ e $\pi(\beta \mid D)$ as densidades de $\beta$ a priori e a posteriori respectivamente, onde, $\mathrm{D}=$ conjunto de dados. Utilizaremos as seguintes densidades a priori de parâmetro $\beta$ :

(i) Densidade a Priori Gama (DPG):

$$
\pi(\beta)=\frac{b^{q}}{\Gamma(q)} \beta^{q-1} e^{-b \beta}, \quad(0<\beta<\infty ; b, q>0)
$$

Portanto, $\beta \sim G(q, b)$, onde os hiperparâmetros $(q, b)$ são conhecidos.

(ii) Priori Não-informativa Difusa (PND):

$$
\pi(\beta)=1, \quad(0<\beta<\infty) .
$$




\subsection{ESTIMAÇÃO BAYESIANA PARA A (DPG)}

A fdp conjunta para os dados $D_{1}=\left(x_{i}, t_{i}\right), i=1, \ldots, n$ (com censuras do tipo II) é obtida (ver Kalbfleisch e Prentice, 1980) a partir da equação (3.2), isto é:

$$
\begin{aligned}
f(\underset{\sim}{t} \mid \beta) & =\frac{n !}{(n-d) !} \prod_{i \notin C} f\left(t_{i} \mid \beta\right) \prod_{i \in C} S\left(t_{i} \mid \beta\right) \\
& =\frac{n !}{(n-d) !} \prod_{i=1}^{d} \beta x_{i} \cdot e^{-\beta x_{i} t_{i}} \cdot e^{-\beta x_{d+1} t_{d}} \ldots e^{-\beta x_{n} t_{d}} \\
& =\frac{n !}{(n-d) !}\left[\prod_{i=1}^{d} x_{i}\right] \beta^{d} \cdot e^{-\beta\left[\sum_{i=1}^{d} x_{i} t_{i}+t_{d} \sum_{i=d+1}^{n} x_{i}\right]} \\
& =\frac{n !}{(n-d) !}\left[\prod_{i=1}^{d} x_{i}\right] \beta^{d} \cdot e^{-\beta Q}, \quad\left(0<t_{1}<t_{2}<\cdots<t_{d}<\infty\right), \beta>0
\end{aligned}
$$

onde, $\underset{\sim}{t}=\left(t_{1}, t_{2}, \cdots, t_{d}\right)$, e a estatística $Q$ é definida por:

$$
Q=\sum_{i=1}^{d} t_{i} x_{i}+t_{d} \sum_{i=d+1}^{n} x_{i}
$$

Portanto, o núcleo da função de verossimilhança será dado por :

$$
\ell(\beta) \propto \beta^{d} \mathrm{e}^{-\beta Q} \quad, \quad(0<\beta<\infty)
$$

Combinando a equação (3.7) com a DPG (3.3), obtemos a densidade a posteriori de $\beta$,

$$
\pi(\beta \mid D)=\frac{(b+Q)^{d+q}}{\Gamma(d+q)} \beta^{d+q-1} \exp \{-(b+Q) \beta\}, \quad 0<\beta<\infty
$$

Portanto, $\pi(\beta \mid D) \sim G(d+q, b+Q)$ pertence a família conjugada de densidades a priori gama. 
Sob a suposição da função de perda quadrática, o valor esperado da posteriori (3.8) fornece um estimador de Bayes de $\beta$ dado por:

$$
\hat{\beta}=\int_{0}^{\infty} \beta \cdot \pi(\beta \mid D) d \beta=\frac{(d+q)}{(b+Q)}
$$

A variância a posteriori de $\beta$ é dada por:

$$
V_{\beta}=\frac{(d+q)}{(b+Q)^{2}}
$$

Do mesmo modo, o estimador bayesiano do tempo médio de sobrevivência, para um paciente com variável auxiliar $\mathrm{x}$, é dado por:

$$
\hat{\mu}=E(\mu \mid x)=\int_{0}^{\infty}(\beta x)^{-1} \pi(\beta \mid D) d \beta=\frac{(b+Q)}{x(d+q-1)} .
$$

A variância a posteriori de $\mu$ é dada por:

$$
V_{\mu}=\frac{(b+Q)^{2}}{x^{2}(d+q-1)^{2}(d+q-2)} \quad, \quad d \geq 2
$$

Sob a suposição da função de perda quadrática, o estimador de Bayes da função de sobrevivência é dado por :

$$
\hat{S}=\hat{S}(t \mid x)=\int_{0}^{\infty} e^{-\beta x t} \pi(\beta \mid D) d \beta=\left[1+\frac{x t}{(b+Q)}\right]^{-(d+q)}
$$

A variância a posteriori da função de sobrevivência é obtida como : 


$$
V_{s}=\left[1+\frac{2 x t}{(b+Q)}\right]^{-(d+q)}-\left[1+\frac{x t}{(b+q)}\right]^{-2(d+q)}
$$

Os resultados bayesianos para a priori não-informativa podem ser obtidos escrevendo $\mathrm{b}=0$ e $\mathrm{q}=1$ nas equações (3.9) - (3.14) .

\subsection{ESTIMAÇÃO BAYESIANA PARA AMOSTRAS COM CENSURAS DO TIPO III}

Em muitos estudos clínicos relacionados com dados de análise de sobrevivência, os pacientes entram no estudo em pontos diferentes de medidas de tempo de uma origem comum $\mathrm{t}=0$, e o estudo clínico é encerrado no final de um período de acompanhamento fixado $\mathrm{t}=\mathrm{T}_{0}$.

Durante o período total $\mathbf{T}_{\mathbf{0}}$ de acompanhamento, alguns pacientes podem morrer, abandonar ("perdidos") ou sobreviver. Se o número total de pacientes que entraram para o estudo clínico é denotado por $\mathbf{n}$ e o número de pacientes que morreram antes do final do período de acompanhamento $T_{0}$ é denotado por $\mathbf{d}$, então o restante dos pacientes $\mathbf{s}=(\mathbf{n}$-d) consiste de pacientes que abandonaram o acompanhamento em tempos diferentes e daqueles que sobreviveram após o fim do estudo. Portanto, o conjunto de dados consiste no vetor de observações $\left(x_{j}, t_{j}\right), j=1, \ldots, d e\left(x_{k}, t_{k}\right), k=1,2, \ldots, s$, onde $t_{j}$ representa o tempo até a morte do $\mathrm{j}$-ésimo paciente, $\mathrm{x}_{\mathrm{j}}$ é o valor da variável auxiliar $\mathrm{x}$ para o $\mathrm{j}$-ésimo paciente, $\mathrm{t}_{\mathrm{k}}$ denota o tempo de sobrevivência registrado do k-ésimo paciente $\mathrm{e}_{\mathrm{k}}$ denota o valor registrado da variável auxiliar x para o k-ésimo paciente. Denotaremos este conjunto de dados por $\mathrm{D}_{2}$. Este tipo de censura é conhecido como censura amostral do tipo III ( Lee,1992) ou censura aleatória. Diz-se também que a amostra é progressivamente censurada. 
Para o conjunto de dados $\mathrm{D}_{2}$ descrito acima, a função de verossimilhança pode ser indicada como:

$$
L(\beta) \propto \beta^{d} e^{-\beta Q} \quad, \quad(0<\beta<\infty)
$$

onde,

$$
Q^{*}=\sum_{j=1}^{d} x_{j} t_{j}+\sum_{k=1}^{s} x_{k}^{\prime} t_{k}
$$

A equação (3.15) pode ser combinada com a DPG (3.3) e a PND (3.4), respectivamente, usando o Teorema de Bayes para obter as respectivas densidades a posteriori . Felizmente, todos os resultados bayesianos da seção (3.3) podem ser obtidos para o conjunto de dados $\mathrm{D}_{2}$, substituindo naqueles resultados $\mathrm{Q}$ por $\mathrm{Q}^{*}$. 


\subsection{ILUSTRAÇÕES NUMÉRICAS E GRÁFICAS}

Para ilustrar o uso do modelo (2.1), caracterizado pela taxa de risco $\theta=\beta \times$ (3.1), utilizamos os dados da Tabela 2.4 da seção 2.2. Este conjunto de dados, também analisado por Bhattacharya et al (1995) resulta em uma amostra censurada do tipo III $\operatorname{com} \mathrm{n}=17, \mathrm{~d}=12$, $\mathrm{s}=5$ e fornece o valor $\mathrm{Q}^{*}=3341.11$.

$O$ intervalo de credibilidade $\left[t_{1}, t_{2}\right] 100(1-\alpha) \%,(0<\alpha<1)$ para o tempo de sobrevivência $\mathrm{T}$ é definido pela equação:

$$
P\left\{t_{1} \leq T \leq t_{2}\right\}=\int_{t_{1}}^{t_{2}} f(t \mid D) d t=1-\alpha
$$

onde,

$$
f(t \mid D)=\int_{0}^{\infty} f(t \mid \beta) \pi(\beta \mid D) d \beta=x \frac{(b+Q)^{d+q}(d+q)}{(x t+b+Q)^{d+q+1}} \quad . \quad \text { Note }
$$

que $f(t \mid D)$ é do mesmo tipo de uma distribuição de Pareto.

Assumindo uma densidade a priori Gama $\mathrm{G}(2,1)$, temos a seguinte densidade a posteriori:

$$
\pi(\beta \mid D)=3.484 \times 10^{39} \beta^{13} \mathrm{e}^{-3342.11 \beta} \quad, \quad(0<\beta<\infty)
$$

A Figura 3.1 a seguir mostra os gráficos para estas duas densidades .

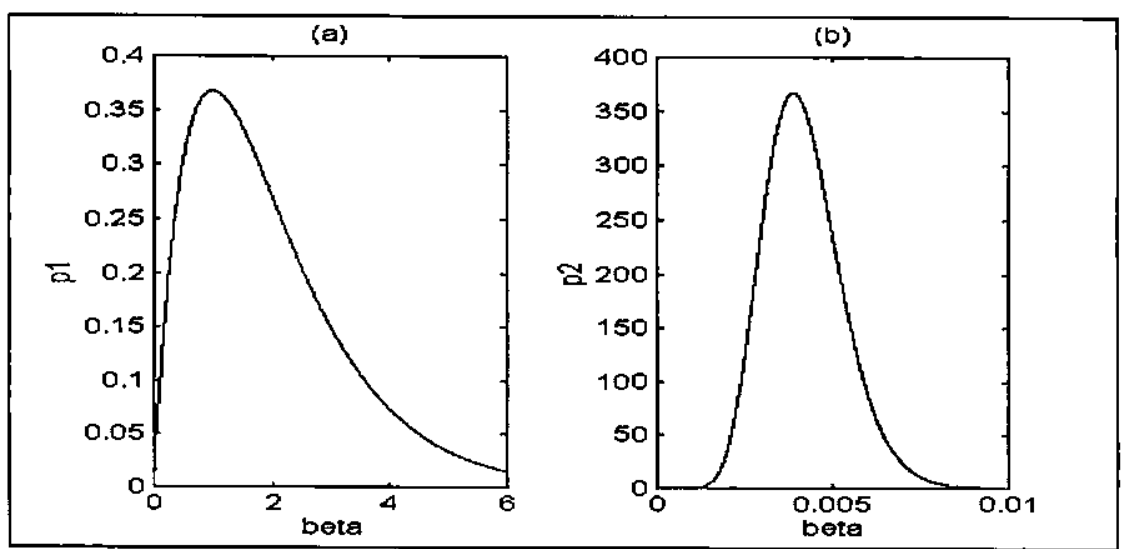

FIGURA 3.1 : Gráfico das densidades em (a) priori Gama(2,1), e em (b) posteriori $\operatorname{Gama}(14,3342.11)$. 
A estimativa bayesiana de $\beta$ é dada por $\hat{\beta}=4.19 \times 10^{-3}$, com variância a posteriori, $\mathrm{V}_{\beta}=1.25 \times 10^{-6}$

A estimativa bayesiana do tempo médio de sobrevivência foi encontrada como sendo $\hat{\mu}=\left(257.09 \mathrm{x}^{-1}\right)$ semanas. Para um paciente com um valor baixo da variável auxiliar $\mathrm{x}=2.88$, temos $\hat{\mu}=89.27$ semanas, enquanto para um valor alto $x=5$, temos $\hat{\mu}=51.42$ semanas. As estimativas bayesianas do tempo médio de sobrevivência para diferentes valores de $\mathrm{x}$ estão em concordância com as correspondentes EMV's , como mostra a Tabela 3.1 a seguir.

TABELA 3.1- Estimativas do tempo médio de sobrevivência (em semanas).

\begin{tabular}{c|c|c|c}
\hline \multirow{2}{*}{$\mathbf{x}$} & \multicolumn{3}{|c|}{ Estimativas do tempo médio de sobrevivência } \\
\cline { 2 - 4 } & $\begin{array}{c}\text { Priori Gama } \\
(\mathbf{q}=\mathbf{2}, \mathbf{b}=1)\end{array}$ & $\begin{array}{c}\text { Priori Não } \\
\text { Informativa }(\boldsymbol{\pi}(\boldsymbol{\beta})=1)\end{array}$ & EMV \\
\hline 2.88 & 89.27 & 96.68 & 96.68 \\
\hline 3.20 & 80.34 & 87.01 & 87.01 \\
\hline 3.40 & 75.61 & 81.89 & 81.89 \\
\hline 3.60 & 71.41 & 77.34 & 77.34 \\
\hline 3.80 & 67.65 & 73.27 & 73.27 \\
\hline 4.00 & 64.27 & 69.61 & 69.61 \\
\hline 4.02 & 63.95 & 69.26 & 69.26 \\
\hline 4.10 & 62.70 & 67.91 & 67.91 \\
\hline 4.20 & 61.21 & 66.29 & 66.29 \\
\hline 4.40 & 58.43 & 63.28 & 63.28 \\
\hline 4.80 & 53.56 & 58.01 & 58.01 \\
\hline 5.00 & 51.42 & 55.69 & 55.69 \\
\hline
\end{tabular}

A estimativa bayesiana da função de sobrevivência $S=S(t / x)$ foi encontrada como sendo:

$$
\hat{S}=\hat{S}(t \mid x)=\left[1+\frac{x t}{3342.11}\right]^{-14}
$$

Para $x=2.88$ e $x=5$, respectivamente, os valores de $\hat{S}$ foram calculados para diferentes valores de $t$ na Tabela 3.2, e estão em concordância com as correspondentes EMV's. 
TABELA 3.2 - Estimativas da função de sobrevivência.

\begin{tabular}{|c|c|c|c|c|c|c|}
\hline \multirow{3}{*}{$\begin{array}{c}\text { Tempo } \\
\text { De } \\
\text { Sobrevivência } \\
\text { (em semanas) }\end{array}$} & \multicolumn{6}{|c|}{ Estimativas da Função de Sobrevivência } \\
\hline & \multicolumn{2}{|c|}{$\begin{array}{l}\text { Priori Gama } \\
(q=2, b=1)\end{array}$} & \multicolumn{2}{|c|}{$\begin{array}{c}\text { Priori Não } \\
\text { Informativa }(\pi(\beta)=1)\end{array}$} & \multicolumn{2}{|c|}{ EMV } \\
\hline & $x=2.88$ & $x=5$ & $x=2.88$ & $x=5$ & $x=2.88$ & $x=5$ \\
\hline 10 & 0.8868 & 0.8123 & 0.8944 & 0.8244 & 0.9017 & 0.8356 \\
\hline 20 & 0.7872 & 0.6618 & 0.8007 & 0.6816 & 0.8131 & 0.6983 \\
\hline 30 & 0.6995 & 0.5408 & 0.7176 & 0.5650 & 0.7332 & 0.5835 \\
\hline 40 & 0.6222 & 0.4432 & 0.6436 & 0.4696 & 0.6612 & 0.4876 \\
\hline 50 & 0.5540 & 0.3642 & 0.5778 & 0.3914 & 0.5962 & 0.4074 \\
\hline 60 & 0.4937 & 0.3002 & 0.5192 & 0.3270 & 0.5376 & 0.3405 \\
\hline 70 & 0.4404 & 0.2480 & 0.4669 & 0.2739 & 0.4848 & 0.2845 \\
\hline 80 & 0.3932 & 0.2054 & 0.4202 & 0.2299 & 0.4371 & 0.2377 \\
\hline 90 & 0.3514 & 0.1706 & 0.3786 & 0.1935 & 0.3942 & 0.1986 \\
\hline 100 & 0.3143 & 0.1420 & 0.3413 & 0.1632 & 0.3554 & 0.1660 \\
\hline
\end{tabular}

Para um paciente com $x=2.88$, um intervalo com $90 \%$ de credibilidade, obtido através de (3.17) com priori $\mathrm{G}(2,1)$, para o tempo de sobrevivência é dado por [4.26, 276.88] semanas, enquanto o intervalo correspondente a $x=5$, é $[2.45,159.48]$ semanas. Podemos ainda observar que a priori própria $\mathrm{G}(2,1)$, apresenta neste caso, o mesmo comportamento da priori não informativa.

Para a priori não-informativa PND dada por (3.4), os resultados similares a estes mencionados acima para a DPG, são apresentados a seguir. A densidade a posteriori neste caso é dada por:

$$
\pi(\beta \mid D)=1.349 \times 10^{37} \beta^{12} \mathrm{e}^{-3341.11 \beta}, \quad(0<\beta<\infty)
$$

O gráfico da densidade (3.20) é mostrado pela Figura 3.2 a seguir .

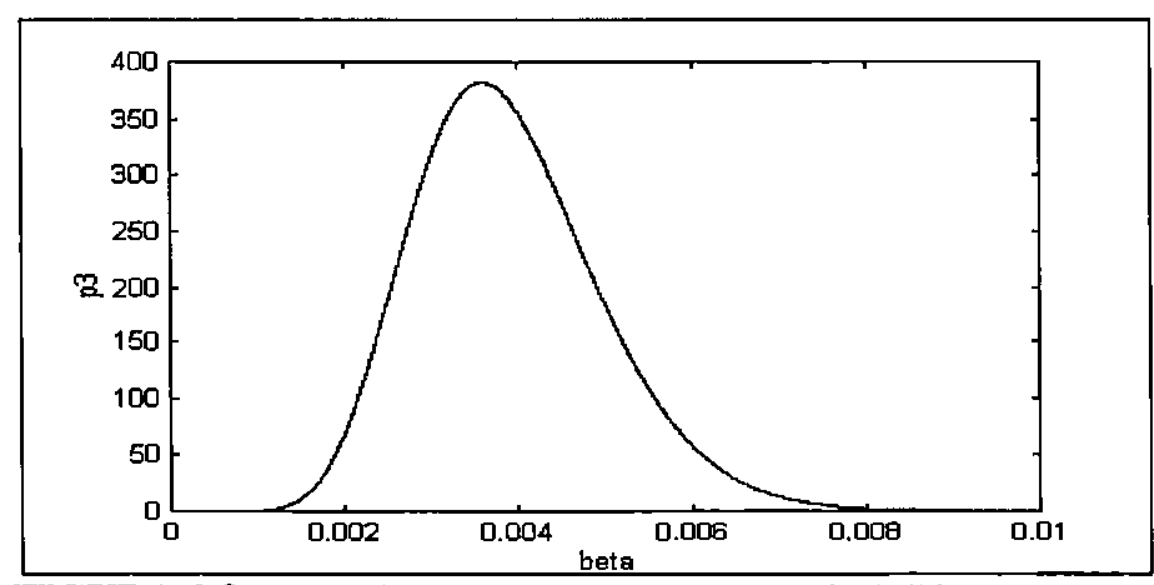

FIGURA 3.2 - Posteriori G(13,3341.11) com priori difusa. 
Podemos observar que os dados amostrais produzem um conhecimento considerável sobre $\beta$. Obtivemos as seguintes estimativas bayesianas: $\hat{\beta}=0.0039, \quad V_{\beta}=1.16 \times 10^{-6}$ e $\hat{\mu}=\left(278.43 \mathrm{x}^{-1}\right)$, de maneira que para $\mathrm{x}=2.88 \mathrm{e} \mathrm{x}=5$, foram encontrados respectivamente $\hat{\mu}=96.68$ e $\hat{\mu}=55.69$ semanas.

A estimativa bayesiana para $\mathrm{S}=\mathrm{S}(\mathrm{t} \mid \mathrm{x})$ com PND foi encontrada como sendo:

$$
\hat{S}=\hat{S}(t \mid x)=\left[1+\frac{x t}{3341.11}\right]^{-13}
$$

Estimativas bayesianas do tempo médio de sobrevivência e da função de sobrevivência para diferentes valores de $\mathrm{x}$ e $\mathrm{t}$ estão em concordância com as correspondentes EMV's (ver Tabelas 3.1 e 3.2 ).

As estimativas das funções de sobrevivência correspondentes para as DPG e PND estão mostradas na Figura 3.3 a seguir, para dois níveis da variável auxiliar $\mathrm{x}=2.88$ e $\mathrm{x}=5$, os quais correspondem ao mínimo e ao máximo da amostra, respectivamente.

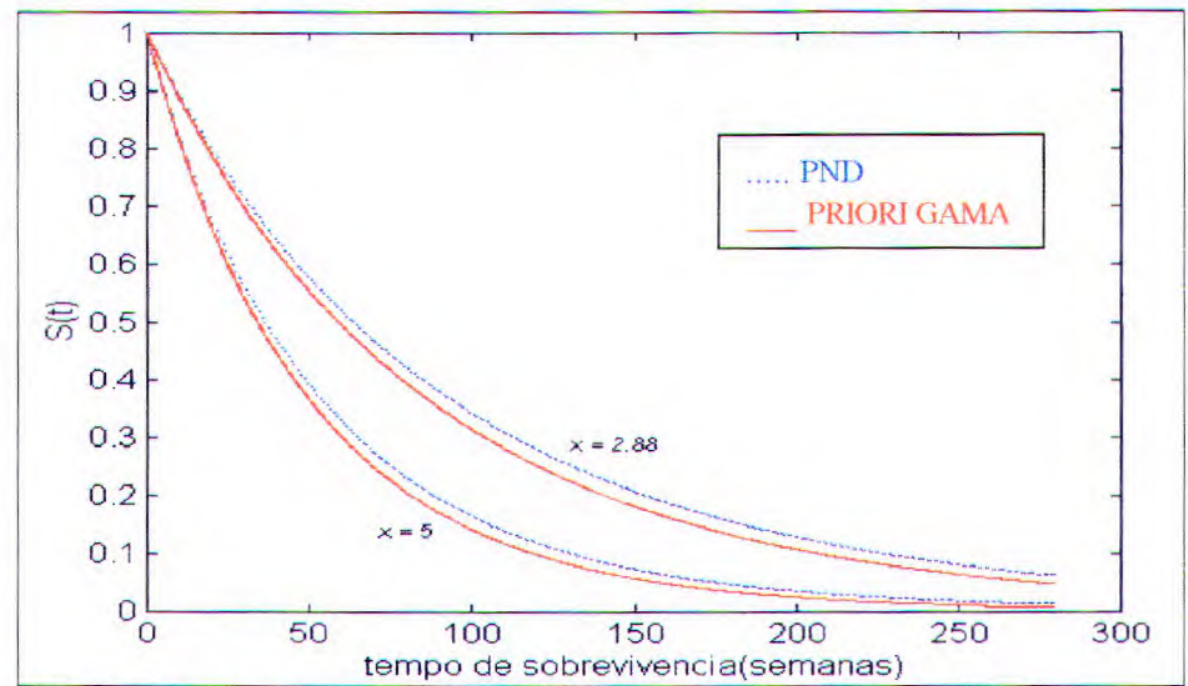

FIGURA 3.3 - Funções de sobrevivência para as DPG e PND, com $x=2.88$ e $x=5$. Note que a priori $G(2,1)$, neste caso, tem comportamento semelhante ao da priori não-informativa sobre a função de sobrevivência.

Podemos ressaltar, que as conclusões apresentadas acima poderiam ser mais precisas se fosse usado uma função de ligação mais geral, por exemplo, $\theta=\alpha+\beta x \quad(\alpha, \beta>0)$

Um desenvolvimento teórico para esta função de ligação entre outras será apresentado no próximo capítulo. 


\section{Capítulo 4}

\section{FUNÇÕES DE LIGAÇÕES ALTERNATIVAS}

Neste capítulo, desenvolvemos uma análise clássica, e bayesiana exata para funções de ligações alternativas às apresentadas nos capítulos anteriores. Na seção 4.1, duas funções de ligações sob o enfoque clássico e na seção 4.2 mais duas outras sugeridas por Bhattacharya et al (1995) sob o enfoque da inferência bayesiana exata.

\subsection{ABORDAGEM CLÁSSICA}

Duas outras formulações do problema são dadas por:

$$
E\left(t_{i}\right)=\left(a+b x_{i}\right)^{-1}
$$

ou

$$
E\left(t_{i}\right)=a e^{b x_{i}}
$$

Quando (4.1) é usada, a estatística $\left(\sum_{\mathrm{i}} \mathrm{t}_{\mathrm{i}}, \sum_{\mathrm{i}} \mathrm{x}_{\mathrm{i}} \mathrm{t}_{\mathrm{i}}\right)$ é uma estatística suficiente para (a , b), como está indicado em Cox (1964).

O modelo dado por (4.2) é equivalente, assumindo-se que o logaritmo do tempo médio de sobrevivência é uma função linear da variável auxiliar. Uma parametrização mais conveniente deste modelo é dada por :

$$
E\left(t_{i}\right)=a e^{b d_{i}}, \quad d_{i}=x_{i}-\bar{x} .
$$

Usando (4.3), a log-verossimilhança pode ser escrita como: 


$$
L(a, b)=-n \log a-\sum_{i} \frac{t_{i} e^{-b d_{i}}}{a}
$$

As estimativas de máxima verossimilhança são obtidas pela solução das equações :

$$
\begin{aligned}
& \sum_{i=1}^{n} t_{i} d_{i} e^{-\hat{b} d_{i}}=0 \\
& \hat{a}=\sum_{i=1}^{n} \frac{t_{i} e^{-\hat{b} d_{i}}}{n} .
\end{aligned}
$$

As variâncias e covariâncias assintóticas dos estimadores de máxima verossimilhança são facilmente encontradas, isto é:

$$
\begin{aligned}
& \operatorname{var}(\hat{a})=a^{2} / n, \\
& \operatorname{var}(\hat{b})=\left(\sum_{i=1}^{n} d_{i}^{2}\right)^{-1} \quad, \quad \operatorname{cov}(\hat{a}, \hat{b})=0 .
\end{aligned}
$$

O problema numérico restringe-se a encontrar uma solução para (4.5), a qual pode ser obtida por iteração. Se

$$
\phi(b)=\sum_{i=1}^{n} t_{i} d_{i} e^{-b d_{i}}
$$

e $\hat{b}_{k}$ é a estimativa para a k-ésima iteração, então $\hat{b}_{k+1}$, o valor de $\hat{b}$ na $(k+1)$-ésima iteração, é

$$
\hat{\mathrm{b}}_{\mathrm{k}+1}=\hat{\mathrm{b}}_{\mathrm{k}}-\phi\left(\hat{\mathrm{b}}_{\mathrm{k}}\right) / \phi^{\prime}\left(\hat{\mathrm{b}}_{\mathrm{k}}\right)
$$

onde,

$$
\phi^{\prime}\left(\hat{b}_{k}\right)=-\sum_{i=1}^{n} t_{i} d_{i}^{2} e^{-\hat{b}_{k} d_{i}} .
$$

Uma estimativa inicial para $\hat{b}$ é obtida pelo ajuste de uma reta de mínimos quadrados aos pares $\left(d_{i}, \operatorname{lnt}_{i}\right)$, pois $t_{i}$ satisfaz (4.3), de modo que

$$
t_{i}=a e^{b d_{i}}+e_{i}, \quad \text { onde, } \quad E\left(e_{i}\right)=0 \text {. }
$$




$$
\Rightarrow \quad \ln \mathrm{t}_{\mathrm{i}}=\ln \mathrm{a}+\mathrm{bd} \mathrm{d}_{\mathrm{i}}+\ln \mathrm{e}_{\mathrm{i}}
$$

Portanto,

$$
\hat{\mathrm{b}}_{0}=\frac{\sum_{\mathrm{i}=1}^{n} \mathrm{~d}_{\mathrm{i}}\left(\ln \mathrm{t}_{\mathrm{i}}\right)}{\sum_{\mathrm{i}=1}^{n} \mathrm{~d}_{\mathrm{i}}^{2}} \quad \text { é uma estimativa inicial para } \hat{\mathrm{b}} \text {. }
$$

\subsection{ABORDAGEM BAYESIANA}

Assumiremos novamente o modelo de sobrevivência exponencial (2.1), onde a taxa de risco $\theta$ é dada pela expressão:

$$
\theta=\alpha+\beta x, \quad(\alpha, \beta>0) \quad
$$

A estrutura dos dados de sobrevivência será assumida como $\mathrm{D}_{2}$ (amostra censurada do tipo III). A função de verossimilhança para estes dados amostrais é dada por :

$$
\ell(\alpha, \beta)=\left[\prod_{i=1}^{d}\left(\alpha+\beta x_{i}\right)\right] e^{-\alpha S^{*}-\beta Q^{*}}, \quad(0<\alpha, \beta<\infty)
$$

onde $\mathrm{Q}^{*}$ é dado por (3.16), e $\mathrm{S}^{*}$ é dado pela expressão :

$$
\mathrm{S}^{*}=\left(\sum_{\mathrm{j}=1}^{\mathrm{d}} \mathrm{t}_{\mathrm{j}}\right)+\left(\sum_{\mathrm{k}=1}^{\mathrm{s}} \mathrm{t}_{\mathrm{k}}\right)
$$

a qual representa o tempo de sobrevivência total acumulado dos dados amostrais observados. Se escrevermos o termo produto em (4.10) como uma soma, a função de verossimilhança pode ser reescrita como: 


$$
\ell(\alpha, \beta)=\sum_{r=0}^{d} S_{r, d} \alpha^{d-\tau} \beta^{r} e^{-\alpha S^{*}-\beta Q^{*}}, \quad(\alpha, \beta>0)
$$

onde $S_{0, d}=1, S_{1, d}=\sum_{j=1}^{d} x_{j}$, e para $r=2,3, \ldots, d, S_{r, d}$ é a soma de todos os possíveis produtos de $r$ elementos distintos do conjunto $x=\left\{x_{1}, x_{2}, \ldots, x_{d}\right\}$.

Para as densidades a priori de $\alpha$ e $\beta$, foi assumido que $\alpha \sim G(a, v), \beta \sim G(b, q)$ e que $\alpha$ e $\beta$ são densidades a priori independentes. Portanto, a densidade a priori conjunta de $(\alpha, \beta)$ é dada por :

$$
\pi(\alpha, \beta)=\frac{a^{v} b^{q}}{\Gamma(v) \Gamma(q)} \alpha^{v-1} \beta^{q-1} e^{-a \alpha-b \beta} \quad(\alpha, \beta>0)
$$

onde $a, b, v, q>0$. Assim, a densidade a posteriori conjunta de $(\alpha, \beta)$ é obtida como:

$$
\pi(\alpha, \beta \mid D)=K^{-1} \sum_{r=0}^{d} S_{r, d} \alpha^{d+v-r-1} \beta^{q+r-1} e^{-\alpha\left(a+s^{*}\right)-\beta\left(b+Q^{*}\right)}, \quad(\alpha, \beta>0),
$$

onde

$$
K=\sum_{r=0}^{d} S_{r, d} \frac{\Gamma(d+v-r) \Gamma(q+r)}{\left(a+S^{*}\right)^{d+v-r}\left(b+Q^{*}\right)^{q+r}}
$$

Através de (4.14), as densidades a marginais posteriori de $\alpha$ e $\beta$ são dadas por :

$$
\pi(\alpha \mid D)=K^{-1} \sum_{r=0}^{d} S_{r, d} \frac{\Gamma(q+r)}{\left(b+Q^{*}\right)^{q+r}} \alpha^{d+v-r-1} e^{-\alpha\left(a+s^{*}\right)} \quad(\alpha, \beta>0),
$$

e

$$
\pi(\beta \mid D)=K^{-1} \sum_{r=0}^{d} S_{r, d} \frac{\Gamma(d+v-r)}{\left(a+S^{*}\right)^{d+v-r}} \beta^{q+r-1} e^{-\beta\left(b+Q^{*}\right)} \quad(0<\beta<\infty) .
$$


Sob a suposição da função de perda quadrática, os estimadores bayesianos de $\alpha$ e $\beta$ e as variâncias a posteriori de $\alpha$ e $\beta$ são respectivamente dados pelas seguintes expressões :

$$
\begin{aligned}
& \hat{\alpha}=K^{-1} \sum_{r=0}^{d} S_{r, d} \frac{\Gamma(d+v-r+1) \Gamma(q+r)}{\left(a+S^{*}\right)^{d+v-r+1}\left(b+Q^{*}\right)^{q+r}}, \\
& \hat{\beta}=K^{-1} \sum_{r=0}^{d} S_{r, d} \frac{\Gamma(d+v-r) \Gamma(q+r+1)}{\left(a+S^{*}\right)^{d+v-r}\left(b+Q^{*}\right)^{q+r+1}}, \\
& V_{\alpha}=K^{-1} \sum_{r=0}^{d} S_{r, d} \frac{\Gamma(d+v-r+2) \Gamma(q+r)}{\left(a+S^{*}\right)^{d+v-r+2}\left(b+Q^{*}\right)^{q+r}}-(\hat{\alpha})^{2}, \\
& V_{\beta}=K^{-1} \sum_{r=0}^{d} S_{r, d} \frac{\Gamma(d+v-r) \Gamma(q+r+2)}{\left(a+S^{*}\right)^{d+v-r}\left(b+Q^{*}\right)^{q+r+2}}-(\hat{\beta})^{2} .
\end{aligned}
$$

O estimador bayesiano do tempo médio de sobrevivência $\mu=(\alpha+\beta x)^{-1}$ para este modelo pode ser avaliado como :

$$
\hat{\mu}=\int_{0}^{\infty} \int_{0}^{\infty}(\alpha+\beta x)^{-1} \pi^{*}(\alpha, \beta) \mathrm{d} \alpha \mathrm{d} \beta
$$

onde $\pi(\alpha, \beta \mid D)$ é dado por (4.14). Integrando com respeito a $\beta$ usando ((A.3), Apêndice A), e em seguida integrando com respeito a $\alpha$ usando ((A.4), Apêndice A), obtém-se o seguinte resultado :

$$
\hat{\mu}=K^{-1} \sum_{r=0}^{d} S_{r, d} \frac{\Gamma(q+r) \Gamma(d+v-r){ }_{2} F_{1}\left[d+v-r, 1 ; d+v+q ; 1-\left(b+Q^{*}\right)\left(a+S^{*}\right)^{-1} x^{-1}\right]}{x(d+v+q-1)\left(a+S^{*}\right)^{d+v-r}\left(b+Q^{*}\right)^{Q+r-1}},
$$

o qual é válido para $d+v+q>1$. Do mesmo modo, a variância a posteriori de $\mu$ é obtida por: 


$$
V_{\mu}=K^{-1} \sum_{r=0}^{d} S_{r, d} \frac{\Gamma(q+r) \Gamma(d+v-r){ }_{2} F_{1}\left[d+v-r, 2 ; d+v+q ; 1-\left(b+Q^{*}\right)\left(a+S^{*}\right)^{-1} x^{-1}\right]}{x^{2}(d+v+q-1)(d+v+q-2)\left(a+S^{*}\right)^{d+v-r}\left(b+Q^{*}\right)^{q+r-2}}
$$

$-(\hat{\mu})^{2}$,

a qual é válida para $d+v+q>2$.

O estimador bayesiano da função de sobrevivência $S=S(t \mid x)=\exp [-(\alpha+\beta x) t]$, é dado por :

$$
\hat{S}=\hat{S}(t \mid x)=\int_{0}^{\infty} \int_{0}^{\infty} e^{-(\alpha+\beta x) t} \pi^{*}(\alpha, \beta) d \alpha d \beta
$$

onde $\pi(\alpha, \beta \mid D)$ é dado por (4.14). Calculando a integral dupla, obteremos :

$$
\hat{S}(t \mid x)=K^{-1} \sum_{r=0}^{d} S_{r, d} \frac{\Gamma(d+v-r) \Gamma(q+r)}{\left(a+S^{*}+t\right)^{d+v-r}\left(b+Q^{*}+t \quad x\right)^{q+r}}
$$

Analogamente, a variância posteriori de S é obtida como :

$$
V_{S}=K^{-1} \sum_{r=0}^{d} S_{r, d} \frac{\Gamma(d+v-r) \Gamma\left(q_{0}+r\right)}{\left(a+S^{*}+2 t\right)^{d+v-r}\left(b+Q^{*}+2 t x\right)^{q+r}}-(\hat{S})^{2}
$$




\section{Capítulo 5}

\section{INFERENCIA BAYESIANA COM DENSIDADES A PRIORI OBTIDAS ATRAVÉS DAS VARIÁVEIS AUXILIARES}

Neste capítulo formulamos uma inferência bayesiana para dados clínicos exponenciais com densidades a priori informativas com dados ampliados sob o contexto de modelos lineares generalizados (MLG).

Vários métodos para especificar densidades a priori para MLG têm sidos propostos, alguns dos quais estão baseados na especificação de densidades a priori normais e em densidades a priori difusas. O método que iremos examinar consiste na obtenção de informações a priori para a média das respostas com correspondentes variáveis auxiliares fixas de modo que se possa induzir, através da função de ligação, uma distribuição a priori sobre os coeficientes de regressão, (ver Bedrick et al, 1996, 1997).

\subsection{PRIORI COM MÉDIAS CONDICIONAIS E PRIORI COM DADOS AMPLIADOS}

A idéia de considerar densidades a priori com médias condicionais (PMC's) parte da avaliação das informações a priori em p locações no espaço das variáveis preditoras. Para cada locação, especifica-se uma distribuição a priori para a média das observações. Iremos especificar densidades a priori independentes para as várias locaçōes, embora não seja teoricamente necessário. A priori sobre os coeficientes de regressão $\beta$ é então induzida da PMC. 
Densidades a priori com médias condicionais (PMC's) estão estreitamente relacionadas a priori com dados ampliados (PDA's). Uma PDA, possui a mesma forma da função de verossimilhança, baseada nas " observações a priori" $\left(\tilde{\mathbf{t}}_{i}, \tilde{\mathbf{x}}_{\mathbf{i}}, \tilde{\mathrm{w}}_{\mathrm{i}}: \mathrm{i}=1, \ldots, \mathrm{p}\right)$.

O $\tilde{t}_{\mathrm{i}}$ pode ser determinado quando o consideramos como uma "observação a priori" típica associada com $\tilde{\mathbf{x}}_{\mathbf{i}}$. Os parâmetros pesos $\tilde{\mathrm{w}}_{\mathrm{i}}$ são vistos como um possível número fracionário a priori de observações associadas com $\tilde{\mathrm{t}}_{\mathrm{i}}$. Em geral, a PMC que corresponde a uma PDA depende da função de ligação. Na seção seguinte apresentamos uma introdução aos modelos lineares generalizados (MLG).

\subsection{MODELOS LINEARES GENERALIZADOS}

Uma observação aleatória $\mathrm{T}$ que possui função densidade de probabilidade dada por:

$$
f(t] \theta, \phi ; w)=h(\phi, t, w) \exp \left[\frac{w}{\phi}\{\theta t-r(\theta)\}\right]
$$

Aqui $\mathrm{r}(\cdot) \mathrm{e} h(\cdot, \cdot, \cdot)$ são funções conhecidas, w é um inteiro conhecido e $\theta$ é um parâmetro de escala desconhecido, o parâmetro $\phi$ é assumido conhecido, assim (5.1) pertence a família exponencial. Portanto a variável aleatória $\mathrm{T}$ depende unicamente de $\theta$. A média de $\mathrm{T}$ é dada por:

$$
E(T) \equiv m=\dot{r}(\theta)
$$

onde $\dot{r}(\theta)=\operatorname{dr}(\theta) / d \theta$. A variância de $T$ é dada por, $\operatorname{var}(T)=\ddot{r}(\theta) \phi / w$, onde $\ddot{r}(\theta)$ é a segunda derivada de $r(\theta)$.

A função $\ddot{r}(\theta)$ é escrita frequientemente como uma função de $m$,

$$
V(m) \equiv \ddot{r}(\theta)=\ddot{r}\left(\dot{r}^{-1}(m)\right) \text {. }
$$

$\mathrm{V}(\mathrm{m})$ é chamada de função de variância. A estrutura linear para distribuições da forma (5.1) é especificada por uma função de ligação, 


$$
g(m)=x^{\prime} \beta,
$$

onde $\beta$ é um vetor de $\mathrm{p}$ coeficientes de regressão desconhecidos, $\mathbf{x}$ é um vetor de $\mathrm{p}$ de variáveis auxiliares conhecidas, e $\mathrm{g}(\cdot)$ é uma função monótona conhecida.

Um MLG consiste em observações independentes $T_{i}, i=1, \ldots, n$ com

$$
\mathrm{T}_{\mathrm{i}} \sim \mathrm{f}\left(\mathrm{t}_{\mathrm{i}} \mid \theta_{\mathrm{i}}, \phi ; \mathrm{w}_{\mathrm{i}}\right), \quad \mathrm{E}\left(\mathrm{T}_{\mathrm{i}}\right) \equiv \mathrm{m}_{\mathrm{i}}, \quad \mathrm{g}\left(\mathrm{m}_{\mathrm{i}}\right)=\mathbf{x}_{\mathrm{i}}^{\prime} \beta
$$

(ver por exemplo, McCullagh e Nelder 1989). Se $\mathrm{g}\left(\mathrm{m}_{\mathrm{i}}\right)=\theta_{\mathrm{i}}$, então o modelo é um MLG canônico e $\mathrm{g}(\cdot)$ é a função de ligação canônica.

A função de verossimilhança para um MLG é

$$
L(\beta)=\left\{\prod_{i=1}^{n} h\left(\phi, t_{i}, w_{i}\right)\right\} \exp \left[\sum_{i=1}^{n} \frac{w_{i}}{\phi}\left\{\theta_{i} t_{i}-r\left(\theta_{i}\right)\right\}\right],
$$

onde $\theta_{i}$ é obtido como uma função de $\beta$ por (5.2) e (5.4), isto é,

$$
\theta_{\mathrm{i}}=\dot{\mathrm{r}}^{-1}\left(\mathrm{~m}_{\mathrm{i}}\right)=\dot{\mathrm{r}}^{-1}\left(\mathrm{~g}^{-1}\left(\mathbf{x}_{\mathbf{i}}^{\prime} \beta\right)\right) \equiv \mathrm{k}\left(\mathbf{x}_{\mathbf{i}}^{\prime} \beta\right)
$$

Serão apenas considerados modelos nos quais os $m_{i}$ 's determinam unicamente $\beta$.

\subsection{ESPECIFICAÇÃO DA PRIORI}

Seja $\tilde{\mathrm{X}}$ uma matriz $\mathrm{p} \times \mathrm{p}$ não-singular com $\tilde{\mathrm{x}}_{\mathrm{i}}^{\prime}$ na i-ésima linha $\mathrm{i}=1, \ldots, \mathrm{p}$ linearmente independentes. Seja $\pi_{0}(\tilde{\mathrm{m}})$ uma priori própria sobre $\tilde{\mathrm{m}}=\left(\tilde{\mathrm{m}}_{1}, \ldots, \tilde{\mathrm{m}}_{\mathrm{p}}\right)^{\prime}$, onde $\tilde{\mathrm{m}}_{\mathrm{i}}=\mathrm{E}\left(\tilde{\mathrm{t}} \mathrm{I} \tilde{\mathbf{x}}_{\mathrm{i}}\right)$, $\tilde{\mathbf{x}}_{\mathbf{i}}, \mathrm{i}=1, \ldots, \mathrm{p}$. 
Teremos,

$$
\tilde{\mathbf{m}} \equiv \mathbf{G}^{-1}(\tilde{\mathbf{X}} \beta) \quad \text { (vetor de transformações tal que exista }
$$

uma relação 1-1 entre $\beta$ e $\tilde{\mathrm{m}}$ ), portanto,

$$
\beta=\tilde{\mathbf{X}}^{-1} \mathbf{G}(\tilde{\mathbf{m}})
$$

Seja $\pi_{0}(\tilde{\mathbf{m}})$ uma priori própria arbitrária sobre $\tilde{\mathbf{m}}$. A priori induzida sobre $\beta$ é

$$
\pi(\beta)=\pi_{0}\left(\mathbf{G}^{-1}(\tilde{\mathbf{X}} \beta)\right)\left|\mathrm{dG}^{-1}(\tilde{\mathbf{X}} \beta)\right|
$$

Considere que as $\tilde{\mathrm{m}}_{\mathfrak{i}} \mathrm{s}$ podem ser avaliadas independentemente, então,

$$
\pi_{0}(\tilde{\mathbf{m}})=\prod_{\mathrm{i}=1}^{\mathrm{p}} \pi_{0 \mathrm{i}}\left(\tilde{\mathrm{m}}_{\mathrm{i}}\right)
$$

Através de (5.7) a priori induzida é dada por :

$$
\begin{aligned}
& \pi(\beta)=\prod_{i=1}^{p} \pi_{0 i}\left(g^{-1}\left(\tilde{\mathbf{x}}_{i}^{\prime} \beta\right)\right) /\left\{\left|\tilde{\mathbf{X}}^{-1}\right| \prod_{i=1}^{\mathrm{p}} \dot{\mathrm{g}}(\tilde{\mathrm{m}})\right\} \\
& \pi(\beta) \propto \prod_{\mathrm{i}=1}^{\mathrm{p}} \pi_{0 \mathrm{i}}\left(\mathrm{g}^{-1}\left(\tilde{\mathbf{x}}_{\mathbf{i}}^{\prime} \beta\right)\right) / \dot{\mathrm{g}}\left(\mathrm{g}^{-1}\left(\tilde{\mathbf{x}}_{\mathrm{i}}^{\prime} \boldsymbol{\beta}\right)\right),
\end{aligned}
$$

onde $\dot{\mathrm{g}}$ é a primeira derivada de $\mathrm{g}$ em relação a $\beta$.

Uma PDA sobre $\beta$ é uma PMC que tem a mesma forma da verossimilhança (5.5), baseada nas "observações a priori" $\left(\tilde{\mathrm{t}}_{\mathrm{i}}, \tilde{\mathbf{x}}_{\mathrm{i}}, \tilde{\mathrm{w}}_{\mathrm{i}}: \mathrm{i}=1, \ldots, \mathrm{p}\right)$, onde $\tilde{\mathrm{t}}_{\mathrm{i}}$ pode ser determinado considerando-se uma "observação a priori" típica associada ao $\tilde{\mathbf{x}}_{\mathbf{i}}$ com peso $\widetilde{\mathrm{w}}_{\mathbf{i}} \cdot \mathrm{O}$ parâmetro $\widetilde{\mathrm{w}}_{\mathrm{i}}$ é visto como um número fracionário a priori de observações associadas com $\tilde{\mathrm{t}}_{\mathrm{i}}$.

É importante observar que é muito mais fácil obter informações sobre a média das variáveis respostas, $\mathrm{E}(\mathrm{tl} \mathbf{x})=\mathrm{m}$, a qual está na mesma escala dos dados, do que obter conhecimento a priori sobre $\beta$. Uma priori para $\beta$ em tais modelos necessariamente requer obtenção separada de informações, porque o significado de $\beta$ depende da função de ligação. 


\subsection{PROCEDIMENTO BAYESIANO PARA UM MODELO DE REGRESSÃO EXPONENCIAL COM UMA VARIÁVEL AUXILIAR}

Cox, (1964); Feigl e Zelen, (1965); Zippin e Armitage, (1966) utilizaram uma relação linear (função de ligação linear) entre o tempo médio de sobrevivência e algumas características da doença ou variáveis auxiliares. A desvantagem desta relação é que os valores dos coeficientes de regressão estão restritos aos valores do tempo médio de sobrevivência. Bhattacharya et al (1995) utilizaram uma função de ligação inversa, além de considerar uma situação em que o risco do paciente decresce com o aumento da variável auxiliar. Alguns autores na literatura como Kaplan e Meier, 1958; Prentice, 1973; Bedrick et al, (1996) sugerem uma relação exponencial entre a taxa de morte e as variáveis auxiliares (função de ligação logarítmica). Iremos considerar o modelo exponencial para o tempo de sobrevivência de pacientes, inicialmente, com a seguinte função de risco:

$$
h\left(t_{i}\right)=\exp \left\{-\left(\alpha+\beta z_{i}\right)\right\}, \quad i=1, \ldots, n
$$

Portanto, $\quad \log \left(\mathrm{E}\left(\mathrm{t}_{\mathrm{i}}\right)\right)=\alpha+\beta \mathrm{z}_{\mathrm{i}}, \quad$ (ligação logarítmica),

onde $t_{i}$ é o tempo médio de sobrevivência do i-ésimo paciente, $z_{i}=x_{i}-\bar{x}, \quad x_{i}$ a variável auxiliar, e $\alpha$ e $\beta$ parâmetros desconhecidos.

Considere que $\mathrm{n}$ pacientes entram no estudo, e que $\mathbf{r}$ destes morrem e $\mathrm{n}-\mathrm{r}$ ainda permanecem vivos no fim do estudo. Então, $t_{1}, t_{2}, \ldots, t_{r}$, são os tempos de sobrevivência enquanto $t_{r+1}, \ldots, t_{n}$, foram censurados em $t_{r+1}^{o}, \ldots, t_{n}^{o}$, respectivamente. A partir do modelo (5.10), a função de verossimilhança será dada por:

$$
L(\alpha, \beta)=\prod_{i=1}^{r} e^{-\left(\alpha+\beta Z_{i}\right)} \exp \left\{-t_{i} e^{-\left(\alpha+\beta Z_{i}\right)}\right\} \exp \left\{-\sum_{j=r+1}^{n} t_{j}^{o} e^{-\left(\alpha+\beta Z_{j}\right)}\right\}
$$

Nossos objetivos do ponto de vista Bayesiano são estimar a curva de sobrevivência, 


$$
P(T>t \mid \alpha, \beta, X)=\exp \left\{-t e^{-(\alpha+\beta(X-\bar{X}))}\right\}
$$

as densidades marginais a posteriori de $\alpha$ e $\beta$ e quantis destas densidades.

\subsubsection{A PRIORI INDUZIDA SOBRE $(\alpha, \beta)$}

Seguindo o procedimento de médias condicionais a priori (PMC) introduzido na seção (5.3), faremos

$$
\tilde{\mathrm{m}}_{\mathrm{i}}=\mathrm{E}\left(\mathrm{T}_{\mathrm{i}} \mid \alpha, \beta, \tilde{\mathrm{X}}_{\mathrm{i}}\right)=\mathrm{e}^{-\left(\alpha+\beta\left(\tilde{\mathrm{X}}_{\mathrm{i}}-\overline{\mathrm{X}}\right)\right)}, \quad \mathrm{i}=1,2
$$

onde, $\left\{\left(\tilde{\mathrm{t}}_{\mathrm{i}}, \tilde{\mathrm{X}}_{\mathrm{i}}\right), \mathrm{i}=1,2\right\}$ são "dados a priori” e, $\tilde{\mathrm{X}}_{1}$ e $\tilde{\mathrm{X}}_{2}$ vetores linearmente independentes, logo

$$
\left(\begin{array}{l}
\alpha \\
\beta
\end{array}\right)=\left(\begin{array}{ll}
1 & \left(\tilde{\mathrm{X}}_{1}-\overline{\mathrm{X}}\right) \\
1 & \left(\tilde{\mathrm{X}}_{2}-\overline{\mathrm{X}}\right)
\end{array}\right)^{-1}\left(\begin{array}{l}
-\log \left(\tilde{\mathrm{m}}_{1}\right) \\
-\log \left(\tilde{\mathrm{m}}_{2}\right)
\end{array}\right) .
$$

Assumindo que $\tilde{\mathrm{m}}_{\mathrm{i}} \sim \operatorname{InvGamma}\left(\tilde{\mathrm{w}}_{\mathrm{i}}, \tilde{\mathrm{w}}_{\mathrm{i}} \tilde{\mathrm{i}}_{\mathrm{i}}\right), \mathrm{i}=1,2$ e $\tilde{\mathrm{w}}_{\mathrm{i}}$ 's como pesos associados $\operatorname{aos} \tilde{\mathrm{i}}_{\mathrm{i}}$, a priori induzida sobre $(\alpha, \beta) \mathrm{em}(5.12)$ é

$$
\pi(\alpha, \beta) \propto \prod_{i=1}^{2} \mathrm{e}^{-\left(\alpha+\beta \tilde{Z}_{i}\right) \tilde{w}_{i}} \exp \left\{-\tilde{t}_{i} \tilde{w}_{i} e^{-\left(\alpha+\beta \tilde{Z}_{i}\right)}\right\}
$$

Agora, nosso problema consiste na escolha apropriada dos pesos $\tilde{\mathbf{w}}_{\mathrm{i}}, \mathrm{i}=1,2$ sob a avaliação da "informação a priori" $\tilde{\leftarrow}$. 
- Escolhendo os pesos $\widetilde{\mathrm{w}}_{\mathrm{i}}$ :

Se fizermos uma comparação entre a parte não-censurada da função de verossimilhança em (5.11) e a priori induzida sobre $(\alpha, \beta)$, é razoável pensar em $\widetilde{\mathrm{w}}_{\mathrm{i}}=1$, isto é, a informação a priori , $\tilde{\mathrm{t}}_{\mathrm{i}}$, tem o mesmo "peso" como a informação de uma única observação $t_{i}$. É interessante observar que esta particular priori induzida é a mesma obtida por Bedrick et al (1996) chamada de "data augmentation prior" (DAP) para uma amostra não-censurada.

- Avaliando os hiperparâmetros $\tilde{\mathrm{t}}_{\mathrm{i}}$, (Bedrick et al, 1996)

Seja $\tilde{p}_{i}=e^{-t_{i} / \tilde{m}_{i}}$, para uma escolha apropriada de $t_{i}, i=1$, 2. Para valores especificados $\tau_{i}, i=1,2$, determinamos $\gamma_{i}$ tal que

$$
P\left(\tilde{p}_{i} \geq \gamma_{i}\right)=\tau_{i}, \quad i=1,2
$$

Portanto temos que

$$
\tau_{\mathrm{i}}=\mathrm{P}\left(\frac{\tilde{\bar{f}}}{\tilde{\mathrm{m}}_{\mathrm{i}}} \leq-\frac{\tilde{\bar{q}} \log \left(\gamma_{\mathrm{i}}\right)}{\mathrm{t}_{\mathrm{i}}}\right)
$$

onde, $\frac{\tilde{\mathrm{t}}_{\mathrm{i}}}{\tilde{\mathrm{m}}_{\mathrm{i}}} \sim \operatorname{Gama}(1,1)$, e uma solução explícita para $\tilde{\mathrm{t}}_{\mathrm{i}}$ é dada por:

$$
\tilde{\mathrm{i}}_{\mathrm{i}}=\frac{\mathrm{t}_{\mathrm{i}} \log \left(1-\tau_{\mathrm{i}}\right)}{\log \left(\gamma_{\mathrm{i}}\right)}
$$

Caso contrário, podemos calcular duas probabilidades para cada i e assim obter duas equações da forma (5.14), que pode ser resolvida simultaneamente para $\left(\widetilde{w}_{i}, \tilde{t}_{i}\right)$. 


\subsubsection{DENSIDADES A POSTERIORI CONJUNTAS E MARGINAIS}

A partir de (5.11) e (5.13), temos para $\tilde{\mathrm{w}}_{1}=1$, a seguinte densidade a posteriori conjunta para $(\alpha, \beta)$ :

$$
\begin{array}{r}
\pi(\alpha, \beta \mid D) \propto \mathrm{e}^{(\mathrm{r}+2) \alpha+\beta\left(\tilde{\mathrm{Z}}_{1}+\tilde{\mathrm{Z}}_{2}+\sum_{\mathrm{i}=1}^{\mathrm{r}} \mathrm{Z}_{\mathrm{i}}\right)} \exp \left\{-\left(\sum_{\mathrm{i}=1}^{2} \tilde{\mathrm{t}}_{\mathrm{i}} \mathrm{e}^{-\left(\alpha+\beta \tilde{\mathrm{Z}}_{\mathrm{i}}\right)}+\right.\right. \\
\left.\left.+\sum_{\mathrm{i}=1}^{\mathrm{r}} \mathrm{t}_{\mathrm{i}} \mathrm{e}^{-\left(\alpha+\beta \mathrm{Z}_{\mathrm{i}}\right)}+\sum_{\mathrm{i}=\mathrm{r}+1}^{\mathrm{n}} \mathrm{t}_{\mathrm{i}}^{\mathrm{o}} \mathrm{e}^{-\left(\alpha+\beta \mathrm{Z}_{\mathrm{i}}\right)}\right)\right\}
\end{array}
$$

As densidades marginais a posteriori são dadas por:

$$
\begin{aligned}
& \pi(\alpha \mid \beta, D) \propto \exp \{-(r+2) \alpha\} A(\alpha, \beta), \\
& \pi(\beta \mid \alpha, D) \propto \exp \left\{-\left(\sum_{i=1}^{r} z_{i}+\tilde{z}_{1}+\tilde{z}_{2}\right) \beta\right\} A(\alpha, \beta),
\end{aligned}
$$

onde,

$$
A(\alpha, \beta)=\exp \left\{-\left(\sum_{i=1}^{r} t_{i} e^{-\left(\alpha+\beta z_{i}\right)}+\sum_{i=r+1}^{n} t_{i}^{0} e^{-\left(\alpha+\beta z_{i}\right)}+\sum_{i=1}^{2} \tilde{t}_{i} e^{-\left(\alpha+\beta \tilde{z}_{i}\right)}\right)\right\}
$$

Note que estas densidades a posteriori, não possuem uma forma conhecida. Portanto, não podemos utilizar o algoritmo amostrador de Gibbs, como uma maneira de tornar possível qualquer inferência sobre $\alpha$ e $\beta$ (ver por exemplo, Casella e George, 1992). Entretanto as variáveis $\alpha$ e $\beta$ poderão ser geradas usando-se as densidades condicionais (5.16) como medidas de transição nas Cadeias de Markov. Neste caso, podemos utilizar o algoritmo MetropolisHastings (ver por exemplo, Chib e Greenberg, 1995). Mais detalhes sobre estes dois algoritmos, podem ser vistos no Apêndice B. 


\section{Capítulo 6}

\section{VERIFICAÇÃO DA ADEQUAÇÃO DE MODELOS E ANÁLISE DE SENSIBILIDADE}

A verificação do ajuste do modelo é fundamental numa análise estatística. Uma boa análise bayesiana, portanto, deverá incluir no mínimo alguma verificação da adequabilidade do modelo ajustado aos dados e a plausibilidade segundo os objetivos de interesse. Se o modelo está bem ajustado aos dados, qualquer conjunto de dados gerado por este modelo, deverá ser similar aos dados observados ou atuais.

Vários métodos para verificar a adequação do ajuste e seleção ou discriminação de modelos são propostos por vários autores do ponto de vista Clássico e Bayesiano, (ver por exemplo, Carlin e Louis, 1997; Gelman et al, 1995; Gelfand e Dey, 1994).

A seguir apresentaremos dois métodos bayesianos que nos permitem, respectivamente, avaliar a adequação e selecionar modelos.

\subsection{UM PROCEDIMENTO PARA VERIFICAÇÃO DA ADEQUAÇÃO DE MODELOS - NÍVEL DESCRITIVO BAYESIANO $\left(\mathbf{n}_{\mathbf{d}}\right)$}

A técnica básica para verificar o ajuste do modelo consiste em extrair valores simulados da distribuição preditiva a posteriori (réplicas da amostra) e comparar estas amostras com os dados observados. Qualquer diferença sistemática entre essas réplicas e os dados observados indicam potenciais falhas do modelo. 
A falta de ajuste dos dados com respeito a distribuição preditiva a posteriori pode ser avaliada pela probabilidade caudal ou nível descritivo bayesiano $\left(\mathbf{n}_{\mathbf{d}}\right)$, (ver por exemplo, Gelman et al, 1995), definido como a probabilidade que os dados replicados sejam mais extremos que os dados observados, segundo uma estatística de teste.

Consideremos as seguintes

\title{
NOTAÇÕES :
}

\author{
y: Dados observados; \\ $\theta \quad$ : Vetor de parâmetros;
}

$\mathrm{y}^{\text {rep }}$ : Cópia dos dados (réplicas). A réplica é o y que poderia ser observado futuramente se o experimento fosse repetido com o mesmo modelo e valor de $\theta$ que originou os dados atuais (y). As réplicas serão obtidas por simulação.

$$
\begin{aligned}
& \mathrm{p}\left(\mathrm{y}^{\text {rep }} \mid \mathrm{y}\right)=\int \mathrm{p}\left(\mathrm{y}^{\text {rep }} \mid \theta\right) \mathrm{p}(\theta \mid \mathrm{y}) \mathrm{d} \theta: \text { Densidade preditiva de } \mathrm{y}^{\mathrm{rep}} ; \\
& \mathrm{T}(\mathrm{y}, \theta): \text { Estatística do teste. }
\end{aligned}
$$

Utilizando-se as notações acima, temos os seguintes resultados:

$$
\begin{aligned}
n_{d} & =P\left(T\left(y^{\text {rep }}, \theta\right) \geq T(y, \theta) \mid y\right) \\
& =\iint I_{T\left(y^{\text {rep }}, \theta\right) \geq T(y, \theta)} p\left(y^{\text {rep }}, \theta \mid y\right) d \theta d y^{\text {rep }} \\
& =\iint I_{T}{\left(y^{\text {rep }}, \theta\right) \geq T(y, \theta)} p(\theta \mid y) p\left(y^{\text {rep }} \mid \theta\right) d \theta d y^{\text {rep }} .
\end{aligned}
$$

Note que a estatística do teste $T(y, \theta)$ pode ser função de parâmetros desconhecidos na abordagem bayesiana, pois são obtidos através de simulações da distribuição a posteriori destes parâmetros.

$O$ resultado (6.1), sugere o seguinte procedimento para calcular $\mathbf{n}_{\mathbf{d}}$ via algoritmos de simulação Monte Carlo com Cadeia de Markov (MCMC) (ver, Gelman et al, 1995): 
- $\operatorname{Gerar} \theta^{(\ell)}$ via $\mathrm{p}(\theta \mid \mathrm{y})$ e em seguida gerar $\mathrm{y}^{\mathrm{rep}(\ell)}$ via $\mathrm{p}\left(\mathrm{y} \mid \theta^{(\ell)}\right)$, para $\ell=1, \ldots, \mathrm{L}$;

- Uma estimativa para $\mathbf{n}_{\mathbf{d}}$ é dada por :

$$
\hat{\mathrm{n}}_{\mathrm{d}}=\frac{\#\left\{\mathrm{~T}\left(\mathrm{y}^{\mathrm{rep}(\ell)}, \theta^{(\ell)}\right) \geq \mathrm{T}\left(\mathrm{y}, \theta^{(\ell)}\right), \ell=1, \ldots, \mathrm{L}\right\}}{\mathrm{L}}
$$

Podemos decidir pela rejeição do modelo, por exemplo, se para alguma estatística de teste $\hat{\mathrm{n}}_{\mathrm{d}}<0.05$ ou $\hat{\mathrm{n}}_{\mathrm{d}}>0.95$. Contudo, devemos chamar a atenção que a escolha da estatística do teste deverá levar em consideração outros aspectos que desejamos verificar como "outliers" e assimetria.

Se temos interesse em verificar o ajuste para valores extremos da distribuição, podemos utilizar

$$
T(y, \theta)=\min \left(y_{i}\right), \quad \text { ou } \quad T(y, \theta)=\max \left(y_{i}\right)
$$

Agora, se o interesse está em verificar a suposição de assimetria, poderemos utilizar,

$$
\left.T(y, \theta)=\mid y_{(90)}-\theta\right)|-| y_{(10)}-\theta \mid
$$

onde $y_{(90)}$ e $y_{(10)}$ correspondem aos percentis 90 e 10 da distribuição, respectivamente. Portanto, dependendo do problema , podemos definir uma estatística apropriada com a finalidade de medir aspectos dos dados que não são levados em consideração pelo modelo.

Uma estatística utilizada com bastante frequêencia como alternativa as anteriores aqui mencionadas é definida por:

$$
T(y, \theta)=\sum_{i} \frac{\left(y_{i}-E\left(y_{i} \mid \theta\right)\right)^{2}}{\operatorname{Var}\left(y_{i} \mid \theta\right)}
$$

É importante observar, como enfatiza Carlin e Louis (1997), que não estamos defendendo o uso do nível descritivo bayesiano na escolha do modelo, porque $\mathbf{n}_{\mathbf{d}}$ não é a probabilidade que o modelo esteja correto dado os dados . Portanto, o nível descritivo bayesiano não poderá ser 
usado para a escolha de modelos concorrentes. No entanto, é uma medida útil para verificar a discrepância entre o modelo proposto e os dados observados.

\subsection{ANÁLISE DE SENSIBILIDADE}

Em situações práticas é freqüente encontrar vários modelos alternativos com um adequado ajuste aos dados. A questão básica é como escolher um desses modelos ou estudar a sensibilidade das inferências sobre esses modelos?

A literatura sobre a escolha Bayesiana de modelos é bastante considerável. Iremos basear nossas decisões, como sugerem Carlin e Louis (1997), utilizando a distribuição preditiva condicional, também referida como preditiva condicional ordenada (CPO),

$$
\begin{aligned}
c_{i}=p\left(y_{i} \mid D_{(i)}\right)=\frac{p(y)}{p\left(D_{(i)}\right)} & =\int p\left(y_{i} \mid \theta, D_{(i)}\right) p\left(\theta \mid D_{(i)} d \theta\right. \\
& =\int p\left(y_{i} \mid \theta\right) p\left(\theta \mid D_{(i)}\right) d \theta \\
& \approx \int p\left(y_{i} \mid \theta\right) p(\theta \mid y) d \theta .
\end{aligned}
$$

Portanto, uma estimativa para $\mathrm{c}_{\mathrm{i}}$ será dada por :

$$
\hat{\mathrm{c}}_{\mathrm{i}}=\hat{\mathrm{p}}\left(\mathrm{y}_{\mathrm{i}} \mid \mathrm{D}_{(\mathrm{i})}\right)=\frac{1}{\mathrm{G}} \sum_{\mathrm{g}=1}^{\mathrm{G}} \mathrm{p}\left(\mathrm{y}_{\mathrm{i}} \mid \theta^{(\mathrm{g})}\right)
$$

onde, $\mathrm{D}_{(\mathrm{i})}=\left\{\mathrm{y}_{1}, \mathrm{y}_{2}, \ldots, \mathrm{y}_{\mathrm{i}-1}, \ldots, \mathrm{y}_{\mathrm{n}}\right\}$. Os valores de $\theta^{(\mathrm{g})}$ são obtidos via amostras MCMC de $p(\theta \mid y)$.

O gráfico de $\hat{p}\left(y_{i} \mid D_{(i)}\right)$ versus i poderá ser utilizado para ilustrar a escolha do modelo e diagnóstico de possíveis "outliers".

Iremos escolher o modelo que maximiza o produto,

$$
\mathrm{c}(\ell)=\prod_{\mathrm{i}=1}^{\mathrm{n}} \mathrm{C}_{\mathrm{i}}(\ell)
$$

onde, $\ell$ indexa os modelos. 


\section{Capítulo 7}

\section{REGRESSÃO EXPONENCIAL BAYESIANA COM UMA VARIÁVEL AUXILIAR - Aplicações}

Iremos utilizar o desenvolvimento teórico dos capítulos 4 e 5 para analisar os dados de Feigl e Zelen (1965), e Zippin e Armitage (1966), assumindo o modelo (2.1) e levando em consideração as funções de ligação logarítmica, inversa e linear. A introdução de censuras na amostra e o tipo de priori irão permitir interessantes comparações com a análise clássica e a inferência exata. A função de sobrevivência preditiva e densidades marginais a posteriori serão as principais ferramentas. A verificação e seleção do modelo será realizada utilizando-se as técnicas do capítulo anterior, isto é , nível descritivo bayesiano e distribuição preditiva condicional ordenada (CPO).

\subsection{INFERÊNCIA PARA A FUNÇÃO DE LIGAÇÃO LOGARÍTMICA}

Consideremos novamente os dados da Tabela 2.4 (Zippin e Armitage, 1966). A função de verossimilhança para o modelo exponencial, com função de risco (5.10), será dada por:

$$
L(\alpha, \beta)=\exp \left\{-12 \alpha-\beta \sum_{i=1}^{12} z_{i}-\sum_{i=1}^{17} t_{i} e^{-\left(\alpha+\beta z_{i}\right)}\right\}
$$

Note que $x_{i}$ foi parametrizada como $z_{i}=x_{i}-\bar{x}$. 


\subsubsection{MODELO COM DADOS CENSURADOS E PRIORI PDA}

Para um modelo de regressão com o preditor, $\log _{10}$ da quantidade inicial de glóbulos brancos no sangue (QGB) mais um intercepto, especificamos informações a priori a dois níveis da QGB. A resposta té o tempo de sobrevivência em semanas para pacientes com leucemia.

Suponha que se possa afirmar, independentemente, que a proporção dos pacientes que sobrevivem além de 1 ano é pelo menos $25 \%$ com probabilidade $90 \%\left(\tau_{1}=0,90\right.$,) para o valor baixo 3 do $\log _{10}$ da QGB, isto é,

$$
\mathrm{P}\left(\exp \left(-\mathrm{t}_{1} / \tilde{\mathrm{m}}_{\mathrm{i}}\right)>0.25\right)=0.90 \text {. }
$$

Suponha também que que a proporção dos pacientes que sobrevivem além de 1 ano é pelo menos $75 \%$ com probabilidade 0,10 para o nível alto 5 da QGB. Logo, fixando $\tilde{x}_{1}^{\prime}=\left[\begin{array}{ll}1 & 3\end{array}\right], \tilde{x}_{2}^{+}=\left[\begin{array}{ll}1 & 5\end{array}\right]$, $\mathrm{t}_{1}=\mathrm{t}_{2}=52, \gamma_{1}=0,25, \tau_{2}=0,10$ e $\gamma_{2}=0,75$, e utilizando (5.14), determina-se os $\tilde{\mathrm{t}}_{\mathrm{i}}$ 's , isto é, $\tilde{\mathrm{t}}_{1}=86,37$, e $\tilde{\mathrm{t}}_{2}=19,04$.

A partir da expressão (5.15), teremos as seguintes densidades marginais a posteriori:

$$
\begin{aligned}
& \pi(\alpha \mid \beta, D) \propto \exp \{-14 \alpha\} A(\alpha, \beta), \\
& \pi(\beta \mid \alpha, D) \propto \exp \left\{-\left(\sum_{i=1}^{12} z_{i}+\tilde{z}_{1}+\tilde{z}_{2}\right) \beta\right\} A(\alpha, \beta),
\end{aligned}
$$

onde,

$$
A(\alpha, \beta)=\exp \left\{-\left(\sum_{i=1}^{17} t_{i} e^{-\left(\alpha+\beta Z_{i}\right)}+86,37 e^{-\left(\alpha+\beta \tilde{Z}_{1}\right)}+19,04 e^{-\left(\alpha+\beta \tilde{Z}_{2}\right)}\right)\right\}
$$

As Figuras 7.1 e 7.2 apresentam as densidades conjuntas a priori e a posteriori e contornos da densidade conjunta. Na parametrização usada, foi subtraído a média do vetor de variáveis auxiliares dos dados observados de ambas variáveis auxiliares atual e a priori. 

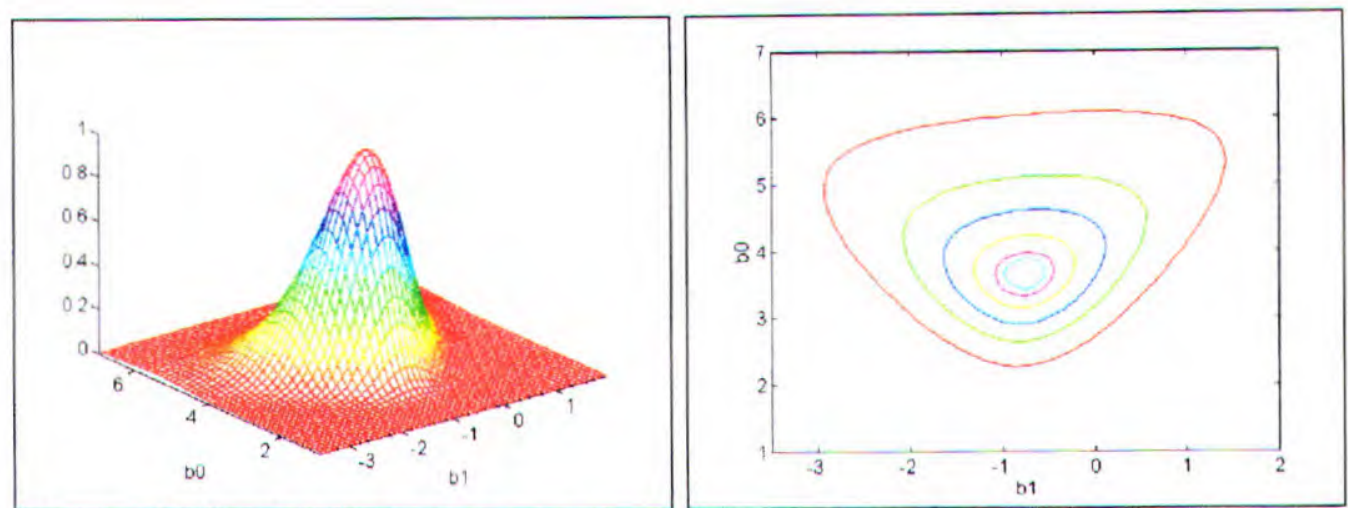

Figura 7.1 - Priori conjunta, e contornos nos niveis 0,$05 ; 0,25$; 0,50 0,75; 0,90 e 0,95. Ligação logarítmica, dados censurados e priori PDA.
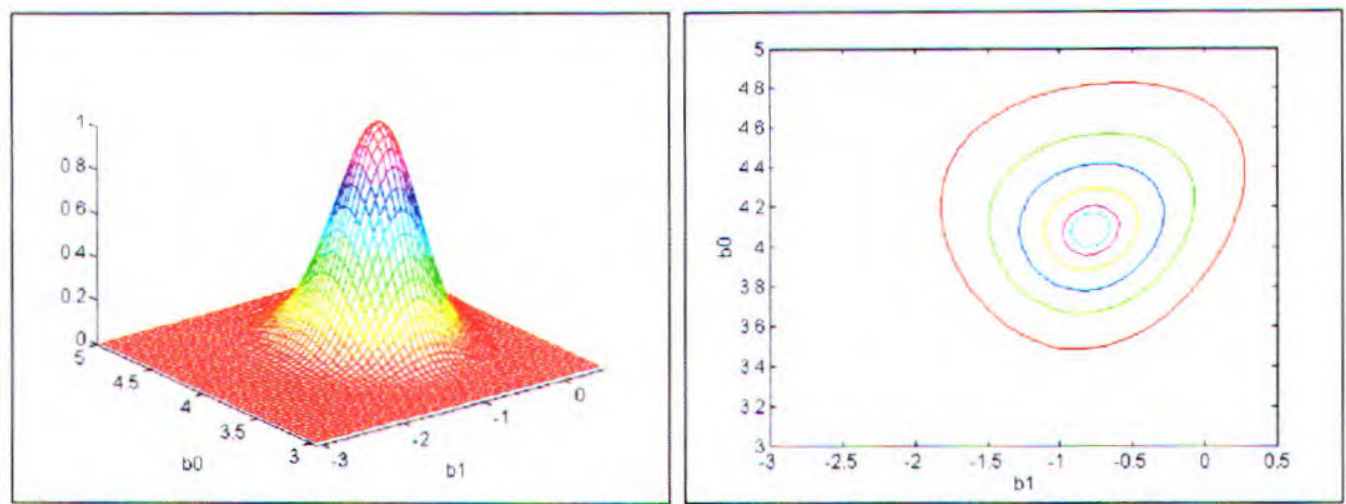

Figura 7.2 - posteriori conjunta, e contornos nos niveis 0,$05 ; 0,25$; 0,50 0,75; 0,90 e 0,95. Ligação logarítmica, dados censurados e priori PDA.

As estimativas das curvas de sobrevivência preditivas a serem apresentadas são obtidas a partir da expressão:

$$
P\left(T \geq t \mid x_{f}, D\right)=\iint \exp \left\{-t e^{-\left(\alpha+\beta x_{f}\right)}\right\} \pi(\alpha, \beta \mid D) d \alpha d \beta
$$

Através das densidades condicionais dadas por (7.2) e (7.3), geramos 5 cadeias de 2000 iterações utilizando o algoritmo Metropolis-Hastings, assumindo núcleo de transição das cadeias para $\alpha$ e $\beta$ normais (ver Apêndice $C$ ). Monitoramos a convergência pelo o critério de Gelman e Rubin (1992) que usa a técnica de análise de variância para determinar se iterações adicionais serão necessárias. Para cada parâmetro, consideramos as iterações $515^{\mathrm{a}}, 530^{\mathrm{a}}, \ldots, 2000^{\mathrm{a}}$. Portanto as 5 cadeias produzem uma amostra de tamanho 500. A seguir, apresentamos um resumo gráfico e algumas estatísticas. 
(a)

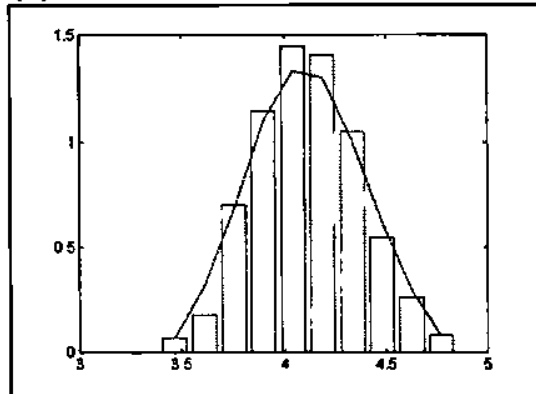

(b)

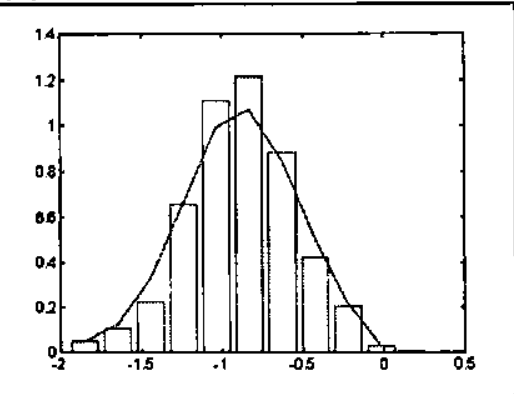

(c)

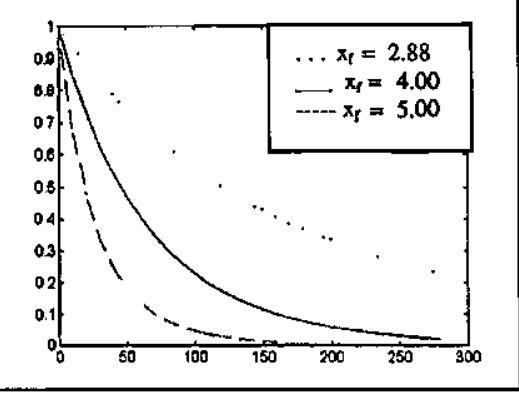

Figura 7.3 - Densidades marginais a posteriori para $\alpha$ em (a), e para $\beta$ em (b), em (c) a distribuição de sobrevivência preditiva. Ligação logarítmica, dados censurados e priori PDA.

As estimativas das três curvas na Figura 7.3 (c) e ao longo deste capítulo foram obtidas utilizando-se os valores $\mathrm{x}_{\mathrm{f}}=2,88 ; 4,00$ e 5,00 os quais representam o mínimo, a mediana e o máximo, respectivamente. Valores obtidos nos dados.

(a)

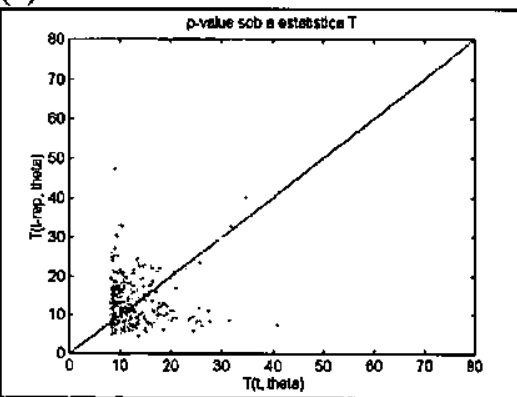

(b)

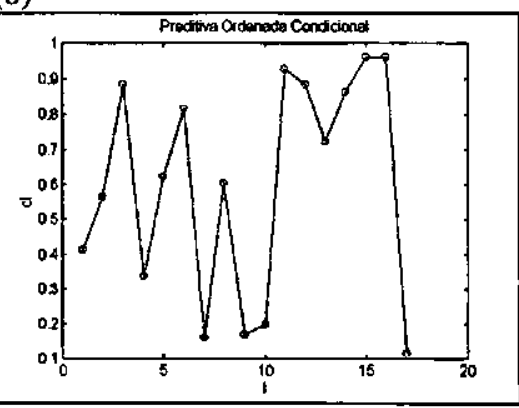

Figura 7.4 - (a) Diagrama de dispersão da estatística do teste,

$\mathrm{T}(\mathrm{y}, \beta)=\sum_{\mathrm{i}} \frac{\left(\mathrm{y}_{\mathrm{i}}-\mathrm{E}\left(\mathrm{y}_{\mathrm{i}} \mid \beta\right)\right)^{2}}{\operatorname{var}\left(\mathrm{y}_{\mathrm{j}} \mid \beta\right)}$, para o nível descritivo bayesiano, $\left(\boldsymbol{n}_{d}=57,8 \%\right)$,

(na abscissa $T(y, \beta))$ versus $\left(T\left(y^{\text {rep }}, \beta\right)\right.$ na ordenada $)$, baseado em 500 simulaçōes.

(b) Preditiva ordenada condicional ( $c_{i}$ versus $i$ ), $c\left(l_{1}\right)=6,384959631162401 \times 10^{-6}$.

(a)

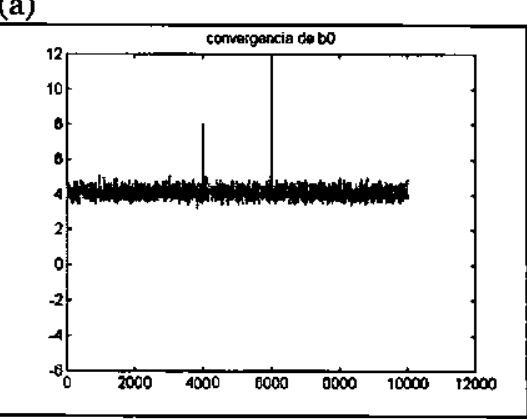

(b)

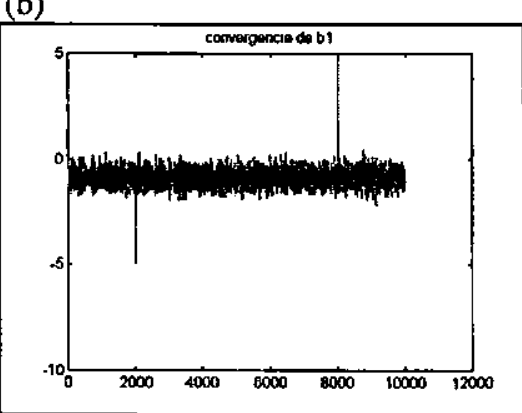

(c)

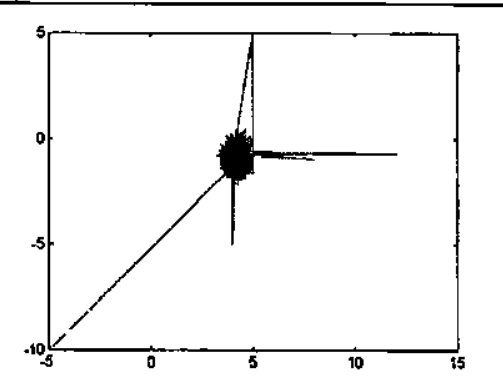

Figura 7.5 - Gráfico da convergência para os parâmetros $\alpha$ e $\beta$ em (a) e (b) respectivamente, e em (c) convergência conjunta, baseados em 10000 simulações 
TABELA 7.1 - Estatísticas a posteriori, ligação logarítimica com priori PDA e dados censurados.

\begin{tabular}{l|c|c}
\hline & $\alpha$ & $\beta$ \\
\hline IC(95\%) & 3,$6355 ; 4,6264$ & $-1,5642 ;-0,2609$ \\
\hline IC(90\%) & 3,$7150 ; 4,5566$ & $-1,4657 ;-3,2987$ \\
\hline Critério de G. e Rubin $(\sqrt{\hat{\mathrm{R}}})$ & 0,998332 & 1,002293 \\
\hline Média & 4,1126 & $-0,8842$ \\
\hline Erro padrão & 0,2576 & 0,3292 \\
\hline Taxa de aceitação & $48,2 \%$ & $68,9 \%$ \\
\hline
\end{tabular}

Na Tabela 7.1 acima temos: Os intervalos de credibilidade (IC) para $\alpha$ e $\beta$ com $95 \%$ e 90\%, note que, ainda para 95\% o limite superior do IC de $\beta$ é negativo; o Critério de G. e Rubin $(\sqrt{\hat{\mathrm{R}}})$, o valor de $\sqrt{\hat{\mathrm{R}}}$ deve estar próximo de 1 para cada parâmetro para se ter indicação de convergência; as estimativas $\alpha$ e $\beta$ pela média das densidades marginais a posteriori; o erro padrão destas estimativas; e a taxa de aceitação dos parâmetros no algoritmo de MetropolisHastings.

\subsubsection{MODELO COM DADOS COMPLETOS E PRIORI PDA}

A seguir apresentamos um resumo gráfico (Figuras 7.6, 7.7 e 7.8) e algumas estatísticas, ambos extraídos de 10000 simulações MCMC para os dados com a amostra completa, priori PDA e função de ligação logarítmica:

(a)

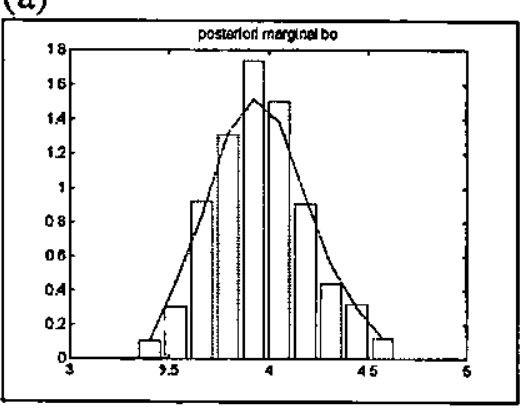

(b)

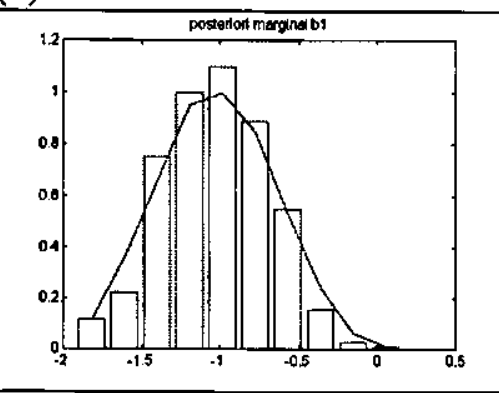

(c)

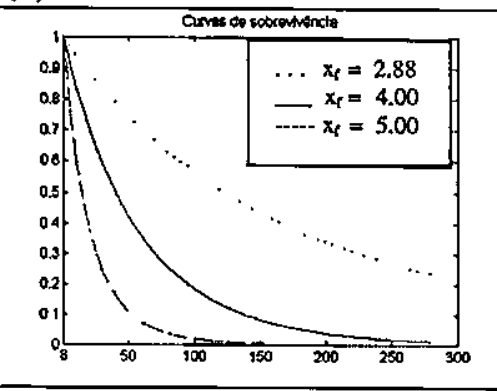

Figura 7.6 - Densidades marginais a Posteriori para $\alpha$ em (a) e para $\beta$ em (b), em (c) a distribuição de sobrevivência preditiva. Gráficos baseados em uma amostra de 500 simulações. Ligaçāo logarítmica, dados completos e priori PDA. 
(a)

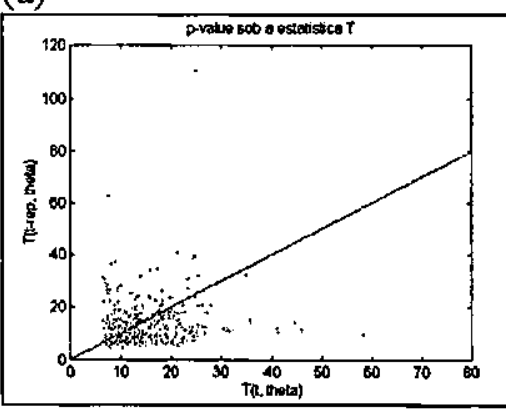

(b)

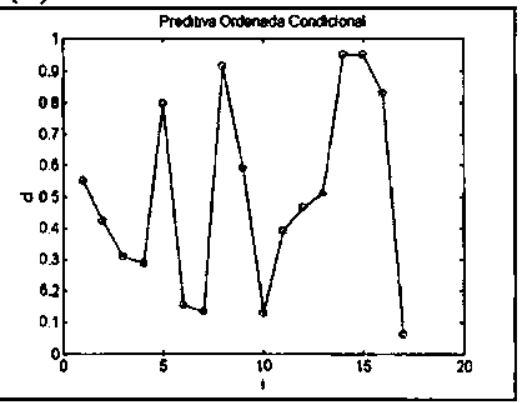

Figura 7.7 - (a) Diagrama de dispersão da estatística do teste, $\mathrm{T}(\mathrm{y}, \beta)=\sum_{\mathrm{i}} \frac{\left(\mathrm{y}_{\mathrm{i}}-\mathrm{E}\left(\mathrm{y}_{\mathrm{i}} \mid \beta\right)\right)^{2}}{\operatorname{var}\left(\mathrm{y}_{\mathrm{i}} \mid \beta\right)}$, para o nivel descritivo bayesiano, $\left(n_{d}=\mathbf{4 6 , 4 \%}\right)$, (na abscissa $T(y, \beta))$ versus $\left(T\left(y^{\text {rep }}, \beta\right)\right.$ na ordenada $)$, baseado em 500 simulações.

(b) Preditiva ordenada condicional $\left(c_{i}\right.$ versus $i$ ). $\mathbf{c}\left(\mathbf{l}_{2}\right)=1,019887140542234 \times 10^{-7}$.

(a)

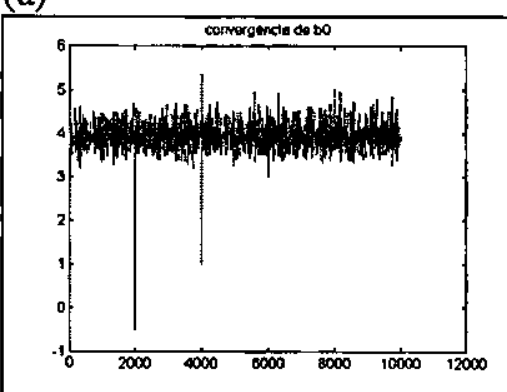

(b)

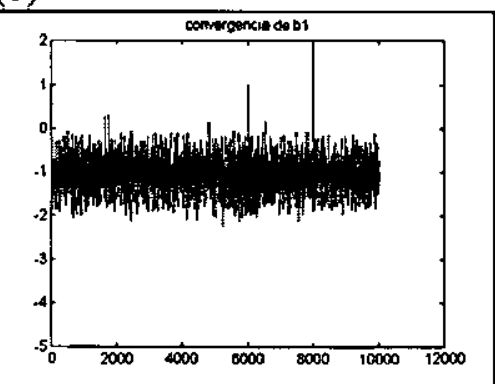

(c)

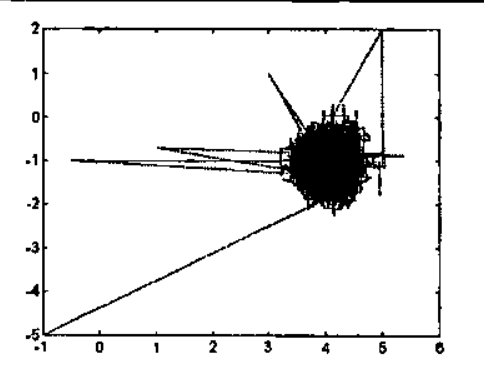

Figura 7.8 - Gráfico da convergência para os parâmetros $\alpha$ e $\beta$ em (a) e (b) respectivamente, e em (c) convergência conjunta, baseados em 10000 simulações.

TABELA 7.2 - Estatísticas a posteriori, ligação logarítmica com priori PDA e amostra completa.

\begin{tabular}{l|c|c}
\hline & $\alpha$ & $\beta$ \\
\hline IC(95\%) & 3,$5262 ; 4,4900$ & $-1,7100 ;-0,4083$ \\
\hline IC(90\%) & 3,$5887 ; 4,3912$ & $-1,5810 ;-0,4874$ \\
\hline Critério de G. e Rubin $(\sqrt{\hat{R}})$ & 1,002729 & 0,998581 \\
\hline Média & 3,9594 & $-1,0277$ \\
\hline Erro padrão & 0,2393 & 0,3372 \\
\hline Taxa de aceitação & $28,2 \%$ & $50,5 \%$ \\
\hline
\end{tabular}




\subsubsection{COMPARANDO O EFEITO ENTRE A AMOSTRA COMPLETA E CENSURADA}

A Tabela 7.3 a seguir, apresenta mais três "amostras a priori" como alternativas a amostra inicial, na intenção de medir o efeito nos resultados a posteriori sob esta situação menos realística. Tendo-se em mente este objetivo, as Figuras 7.9 e 7.10 apresentam gráficos de contornos das densidades a posteriori conjuntas, densidades a posteriori marginais de $\beta$ e curvas de sobrevivência preditiva para as diferentes "amostras a priori" dadas na Tabela 7.3.

TABELA 7.3 - Amostras a priori para $\tau_{1}=0.90$ e $\tau_{2}=0.10$.

\begin{tabular}{c|c|c|c|c|c|c|c}
\hline$\widetilde{\mathrm{x}}_{1}$ & $\widetilde{\mathrm{x}}_{2}$ & $\mathrm{t}_{1}$ & $\mathrm{t}_{\mathbf{2}}$ & $\gamma_{1}$ & $\gamma_{2}$ & $\tilde{\mathrm{t}}$ & $\tilde{\mathrm{t}}_{2}$ \\
\hline $\mathbf{3}$ & $\mathbf{5}$ & $\mathbf{5 2}$ & $\mathbf{5 2}$ & $\mathbf{0 , 2 5}$ & $\mathbf{0 , 7 5}$ & $\mathbf{8 6 , 3 7}$ & $\mathbf{1 9 , 0 4}$ \\
\hline 3 & 5 & 52 & 52 & 0,45 & 0,55 & 149,95 & 9,16 \\
\hline 3 & 5 & 52 & 52 & 0,65 & 0,35 & 277,95 & 5,22 \\
\hline 3 & 5 & 52 & 52 & 0,80 & 0,20 & 536,58 & 3,40 \\
\hline
\end{tabular}

contornos posteriori marginal de $\beta$ curvas de sobrevivência

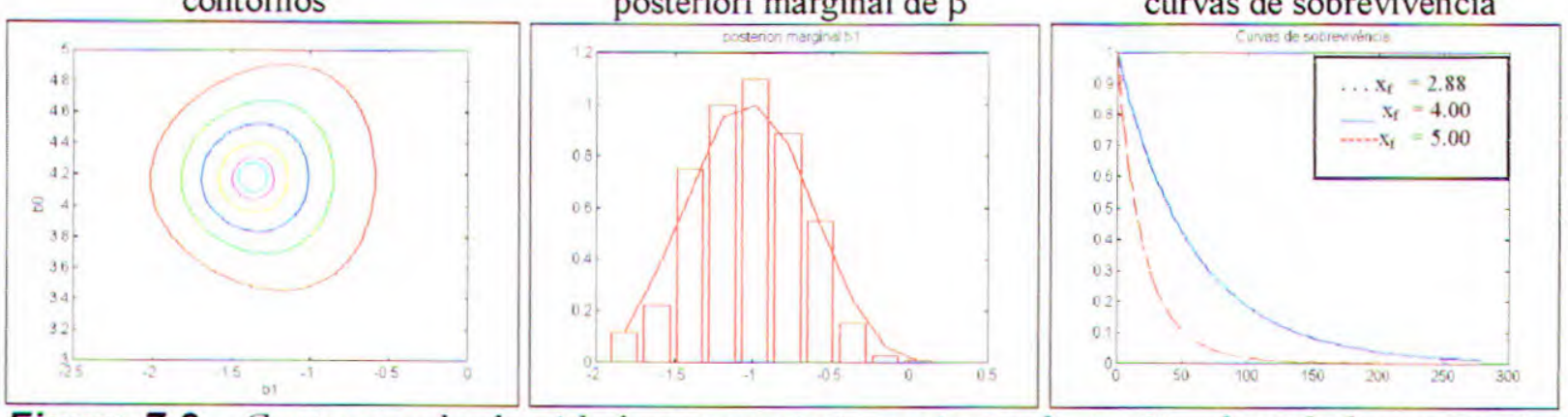

Figura 7.9 - Contornos da densidade a posteriori conjunta de $(\alpha, \beta)$, densidade a posteriori marginal de e curvas de sobrevivencia preditiva. Ligação logaritmica, dados completos e priori PDA. $\widetilde{\mathrm{t}}_{1}=86,37 ; \widetilde{\mathrm{t}}_{2}=19,04 ; \gamma_{1}=0,25 ; \gamma_{2}=0,75$. 
(1) contornos posteriori marginal de $\beta$ curvas de sobrevivência
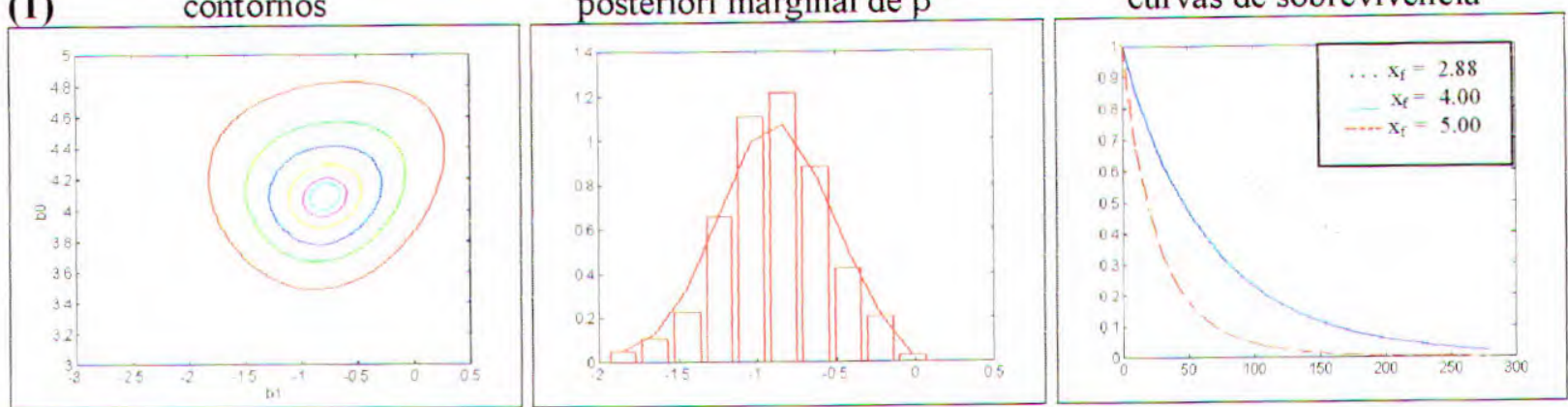

(2) contornos posteriori marginal de $\beta$
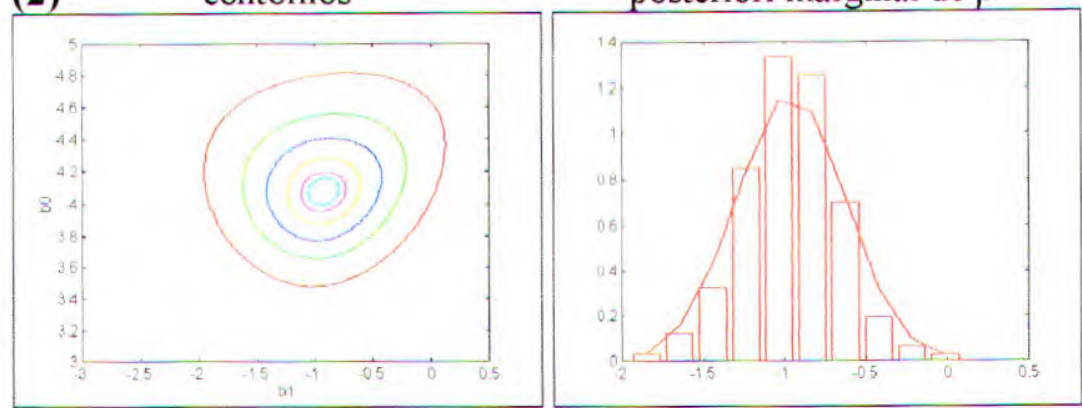

curvas de sobrevivência

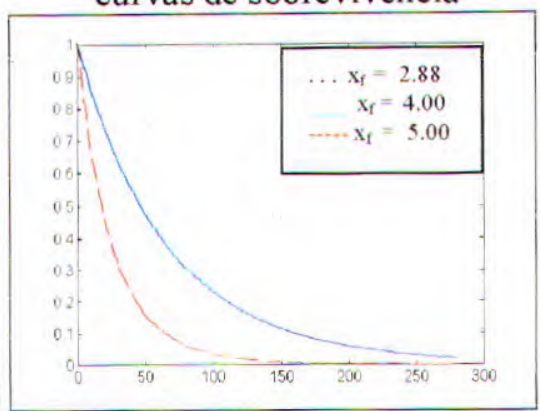

(3) contornos posteriori marginal de $\beta$

curvas de sobrevivência
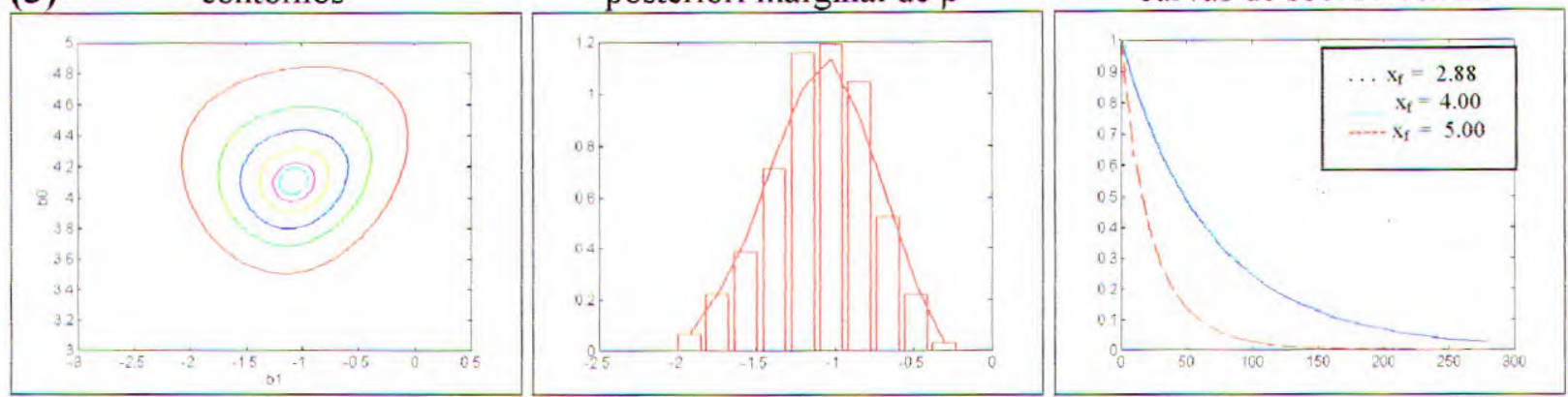

(4) contornos posteriori marginal de $\beta$ curvas de sobrevivência
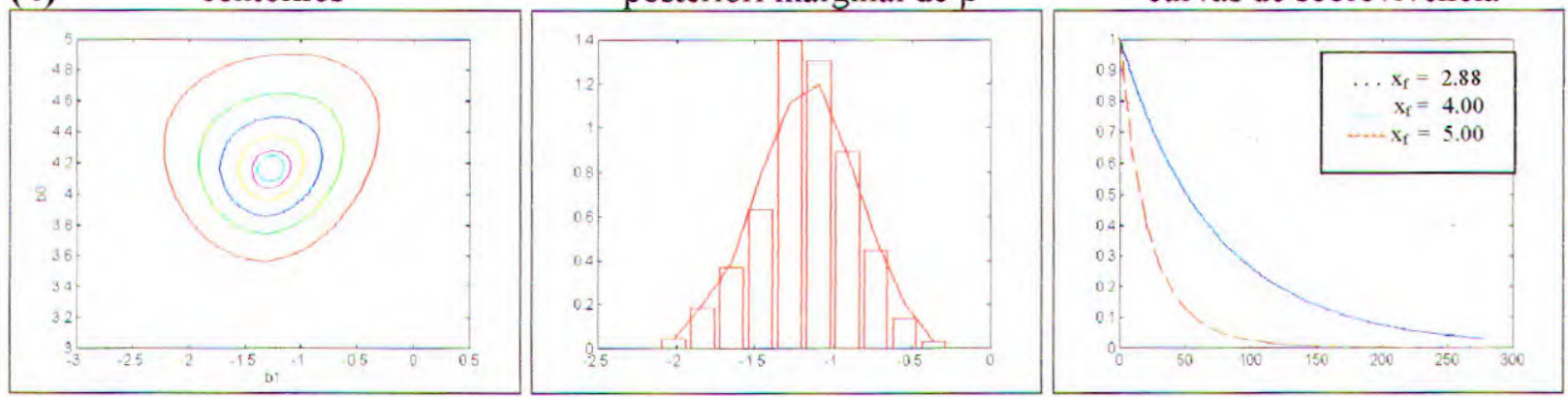

Figura 7.10 - Contornos da densidade a posteriori conjunta de $(\alpha, \beta)$, densidade a posteriori marginal de $\beta$ e curvas de sobrevivência preditiva. Ligação logaritmica, dados completos $e$ priori PDA. (1) $\widetilde{t}_{1}=86,37 ; \widetilde{t}_{2}=19,04 ; \gamma_{1}=0,25 ; \gamma_{2}=0,75 . \quad$ (2) $\widetilde{t}_{1}=149,95 ; \widetilde{t}_{2}=9,16$ $; \gamma_{1}=0,45 ; \quad \gamma_{2}=0,55$. (3) $\tilde{\mathrm{t}}_{1}=277,95 ; \tilde{\mathrm{t}}_{2}=5,22 ; \gamma_{1}=0,65 ; \gamma_{2}=0,35$ e (4) $\tilde{\mathrm{t}}_{1}=536,58$; $\tilde{\mathrm{t}}_{2}=3,40 ; \quad \gamma_{1}=0,80 ; \gamma_{2}=0,20$. 
Podemos comparar os gráficos da Figura 7.10-(1), (2), (3) e (4) para verificar a mudança, sob a presença de censuras, na forma da densidade a posteriori conjunta e densidade marginal de $\beta$, e o deslocamento para cima da distribuição de sobrevivência preditiva correspondente ao nível baixo da QGB. Isto resultaria em previsões mais otimistas do tempo médio de sobrevivência para pacientes com baixa quantidade de globulos brancos. Portanto, este caso reforça a importância da extração da "amostra a priori".

A Tabela 7.4, apresenta quantis da densidade a posteriori de $\beta$ para $\tilde{x}_{1}=3$ e $\tilde{x}_{2}=5$.

TABELA 7.4 - Quantis da densidade a posteriori de $\beta$ com dados censurados.

\begin{tabular}{|c|c|c|c|c|c|c|}
\hline (10) & $\tilde{t}_{2}$ & 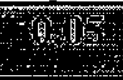 & 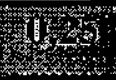 & Hing, & in & 16: \\
\hline 86,37 & 19,04 & $-1,42$ & $-1,11$ & $-0,87$ & $-0,65$ & $-0,32$ \\
\hline 149,95 & 9,16 & $-1,42$ & $-1,14$ & $-0,96$ & $-0,80$ & $-0,51$ \\
\hline 277,95 & 5,22 & $-1,57$ & $-1,28$ & $-1,04$ & $-0,82$ & $-0,49$ \\
\hline 536,58 & 3,40 & $-1,67$ & $-1,40$ & $-1,20$ & $-0,99$ & $-0,71$ \\
\hline
\end{tabular}

Comparando a Figura 7.9 (amostra completa) com a Figura 7.10-(1) (dados censurados), podemos ver claramente um forte efeito dos dados censurados sobre a densidade a posteriori conjunta e distribuição sobrevivência preditiva. 


\subsubsection{MODELO COM DADOS CENSURADOS E PRIORI NÃO- INFORMATIVA DIFUSA $(\pi(\alpha, \beta)=1)$}

Neste caso, a densidade a posteriori conjunta de $\alpha$ e $\beta$ é dada por (7.1).

(a)

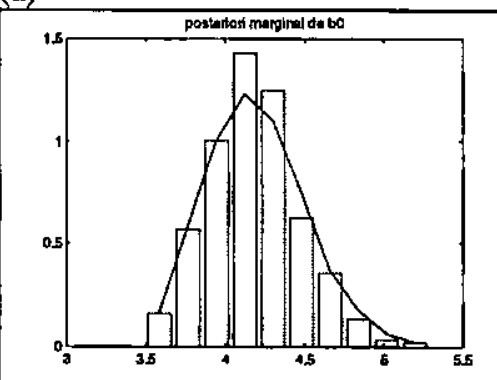

(b)

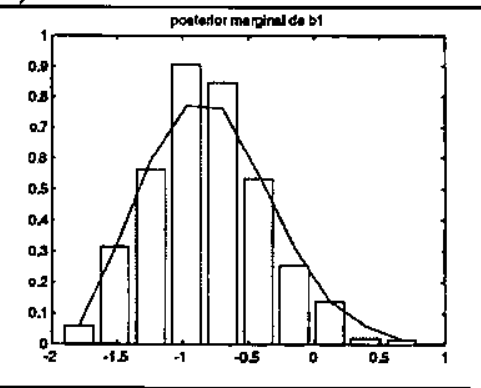

(c)

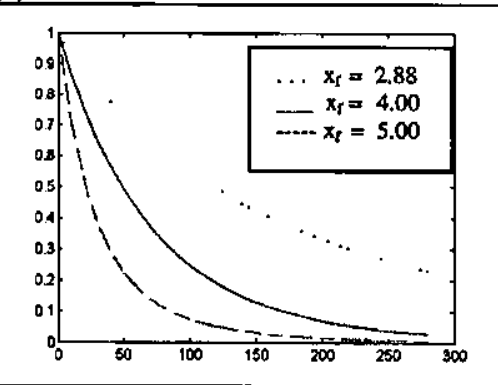

Figura 7.11 -Densidades marginais a posteriori para $\alpha$ em (a), e para $\beta$ em (b), em (c) a distribuição de sobrevivência preditiva. Ligação logarítmica, dados censurados e priori difusa.

(a)

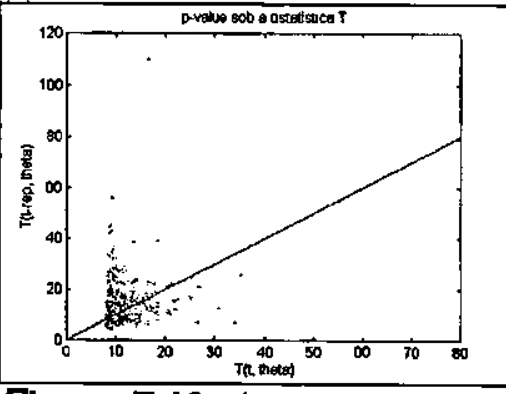

Figura 7.12 - (a) Diagrama de dispersão da estatística do teste,

$\mathrm{T}(\mathrm{y}, \boldsymbol{\beta})=\sum_{\mathrm{i}} \frac{\left(\mathrm{y}_{\mathrm{i}}-\mathrm{E}\left(\mathrm{y}_{\mathrm{i}} \mid \beta\right)\right)^{2}}{\operatorname{var}\left(\mathrm{y}_{\mathrm{i}} \mid \beta\right)}$, para o nível descritivo bayesiano, $\left(\boldsymbol{n}_{d}=\mathbf{5 6 , 4} \%\right)$, (na abscissa

$T(y, \beta))$ versus $\left(T\left(y^{\text {rep }}, \beta\right)\right.$ na ordenada), baseado em 500 simulações.

(b) Preditiva ordenada condicional $\left(c_{i}\right.$ versus $i$ ), $\mathbf{c}\left(\mathrm{I}_{3}\right)=1,3098638130083 \times 10^{-5}$. (a)

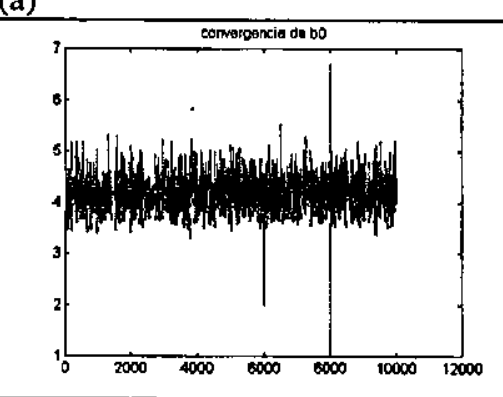

(b)

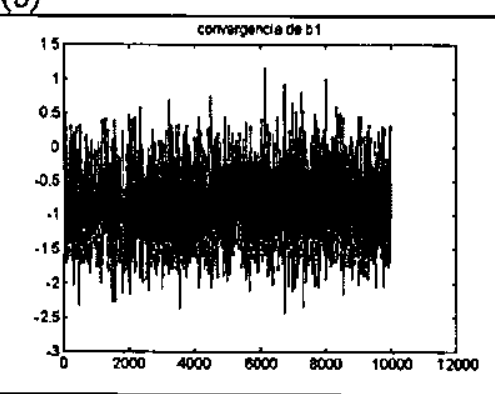

(c)

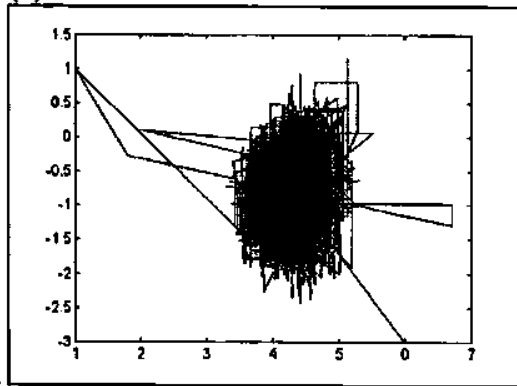

Figura 7.13-Gráfico da convergência para os parâmetros $\alpha$ em (a) e $\beta$ em (b) respectivamente, e em (c) convergência conjunta, baseados em 10000 simulações. 
TABELA 7.5 - Estatísticas a posteriori, com priori difusa, ligação logarítmica e dados censurados.

\begin{tabular}{l|c|c}
\hline & $\alpha$ & $\beta$ \\
\hline $\mathrm{IC}(95 \%)$ & 3,$6552 ; 4,8005$ & $-1.5848 ; 0,1289$ \\
\hline $\mathrm{IC}(90 \%)$ & 3,$7280 ; 4,6605$ & $-1.5082 ;-0,0167$ \\
\hline Critério de G. e Rubin $(\sqrt{\hat{\mathrm{R}}})$ & 1,009003 & 1,001707 \\
\hline Média & 4,1789 & $-0,8154$ \\
\hline Erro padrão & 0,2889 & 0,4418 \\
\hline Taxa de aceitação & $27,7 \%$ & $51,7 \%$ \\
\hline
\end{tabular}

\subsubsection{INFERÊNCIA CLÁSSICA - MODELO COM AMOSTRA CENSURADA}

A função de log-verossimilhança, obtida através da expressão (7.1) é dada por :

$$
\ell(\alpha, \beta)=-12 \alpha-\beta \sum_{i=1}^{12} z_{i}-\sum_{i=1}^{17} t_{i} e^{-\left(\alpha+\beta z_{i}\right)}
$$

As estimativas máxima verossimilhança de $\alpha$ e $\beta$ são dadas pela solução do sistema

$$
S=\left\{\begin{array}{l}
\frac{\partial \ell}{\partial \alpha}=-12+\sum_{i=1}^{17} \mathrm{t}_{\mathrm{i}} \mathrm{e}^{-\left(\alpha+\beta \mathrm{Z}_{\mathrm{i}}\right)}=0 \\
\frac{\partial \ell}{\partial \beta}=-\sum_{\mathrm{i}=1}^{12} \mathrm{z}_{\mathrm{i}}+\sum_{\mathrm{i}=1}^{17} \mathrm{t}_{\mathrm{i}} \mathrm{z}_{\mathrm{i}} \mathrm{e}^{-\left(\alpha+\beta \mathrm{Z}_{\mathrm{i}}\right)}=0
\end{array}\right.
$$

A matriz de variância-covariância assintótica é dada por :

$$
M_{\text {cov }}=-\left[\begin{array}{cc}
E\left(\frac{\partial^{2} \ell}{\partial \alpha^{2}}\right) & E\left(\frac{\partial^{2} \ell}{\partial \alpha \partial \beta}\right) \\
E\left(\frac{\partial^{2} \ell}{\partial \alpha \partial \beta}\right) & E\left(\frac{\partial^{2} \ell}{\partial \beta^{2}}\right)
\end{array}\right]^{-1}=\left[\begin{array}{cc}
\operatorname{var}(\hat{\alpha}) & \operatorname{cov}(\hat{\alpha}, \hat{\beta}) \\
\operatorname{cov}(\hat{\alpha}, \hat{\beta}) & \operatorname{var}(\hat{\beta})
\end{array}\right],
$$


onde,

$$
\begin{aligned}
& E\left(\frac{\partial^{2} \ell}{\partial \alpha^{2}}\right)=-\sum_{i=1}^{17} E\left(t_{i}\right) e^{-\left(\alpha+\beta Z_{i}\right)}=-17 \\
& E\left(\frac{\partial^{2} \ell}{\partial \alpha \partial \beta}\right)=-\sum_{i=1}^{17} E\left(t_{i}\right) z_{i} e^{-\left(\alpha+\beta Z_{i}\right)}=0 \\
& E\left(\frac{\partial^{2} \ell}{\partial \beta^{2}}\right)=-\sum_{i=1}^{17} z_{i}^{2} .
\end{aligned}
$$

Utilizando o método de Newton-Raphson para a solução do sistema de equações (7.7) e calculando (7.8), (ver Apêndice C), teremos a seguir os gráficos da Figura 7.14 e a Tabela 7.6 com algumas estatísticas.

(a)

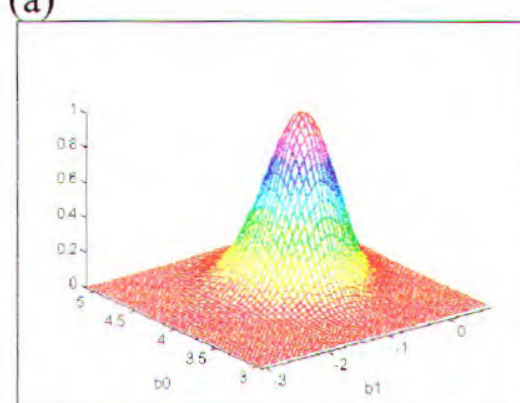

(b)

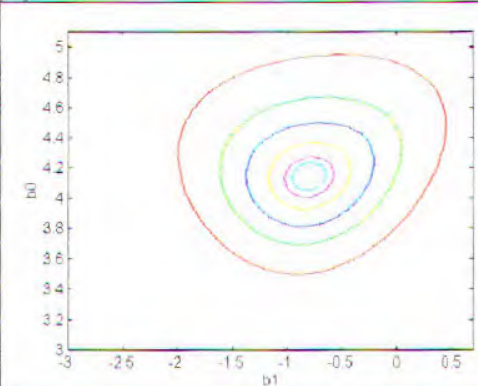

(c)

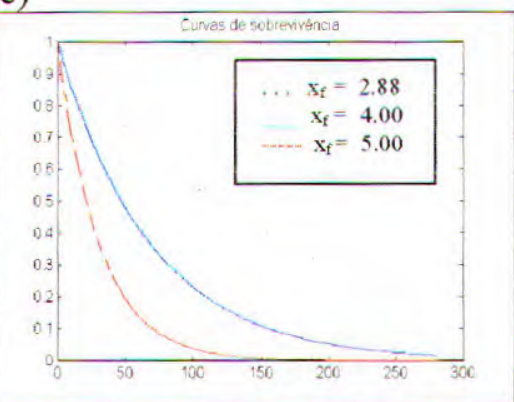

Figura 7.14 - Inferência clássica com dados censurados e ligação logarítmica. (a) Função de verossimilhança relativa, (b) Contornos da função de verossimilhança relativa, (c) Distribuição de sobrevivência preditiva.

TABELA 7.6 - Estatísticas clássicas com ligação logaritmica e dados censurados.

\begin{tabular}{l|c|c}
\hline & $\alpha$ & $\beta$ \\
\hline $\mathbf{I C}(95 \%)$ & 3,$5655 ; 4,7076$ & $-1,7678 ; 0,1701$ \\
\hline IC(90\%) & 3,$6573 ; 4,6158$ & $-1,6120 ; 0,0143$ \\
\hline EMV & 4,1365 & $-0,7988$ \\
\hline Erro padrão & 0,2914 & 0,4944 \\
\hline
\end{tabular}

Comparando a Tabela 7.6 com a Tabela 7.5 , podemos ver que as estimativas pontuais para $\alpha$ e $\beta$ estão compatíveis, o que se pode confirmar, dada a forma simétrica da função de verossimilhança relativa próximo à moda. Quanto aos intervalos, existe uma certa discrepância entre as estimativas clássicas e bayesianas, devido a assimetria existente da função de verossimilhança para valores afastados do centro. 


\subsection{INFERÊNCIA PARA A FUNÇÃO DE LIGAÇÃO INVERSA}

Para o modelo exponencial (2.1), considere que o risco seja dado por:

$$
\theta_{\mathrm{i}}=\alpha+\beta \mathrm{x}_{\mathrm{i}}
$$

isto é,

$$
\mathrm{E}\left(\mathrm{t}_{\mathrm{i}}\right)=\left(\alpha+\beta \mathrm{x}_{\mathrm{i}}\right)^{-1} \quad \text { (ligação inversa). }
$$

Note que não usamos aqui a parametrização $z_{i}=x_{i}-\bar{x}$.

A função de verossimilhança pode ser escrita na seguinte forma:

$$
L(\alpha, \beta)=\prod_{i=1}^{12}\left(\alpha+\beta x_{i}\right) \cdot \exp \left\{-\sum_{j=1}^{17} t_{j}\left(\alpha+\beta x_{j}\right)\right\}
$$

\subsubsection{MODELO COM DADOS CENSURADOS E PRIORI PDA}

Assumiremos como válidas as mesmas considerações feitas para a extração da priori da subseção 5.1.1. Portanto, temos a seguinte priori induzida:

$$
\pi(\alpha, \beta) \propto \exp \left\{-\tilde{\mathrm{t}}_{1}(\alpha+3 \beta)-\tilde{\mathrm{t}}_{2}(\alpha+5) \beta\right\}
$$

Através de (7.10) e (7.11), temos as seguintes densidades marginais a posteriori:

$$
\begin{aligned}
& \pi(\alpha \mid \beta, D) \propto \prod_{i=1}^{12}\left(1+\frac{\beta}{\alpha} x_{i}\right) \alpha^{12} \exp \left\{-\left(\sum_{j=1}^{17} t_{j}+\tilde{t}_{1}+\tilde{t}_{2}\right) \alpha\right\} \\
& \pi(\alpha \mid \beta, D) \propto \prod_{i=1}^{12}\left(\frac{\alpha}{\beta}+x_{i}\right) \beta^{12} \exp \left\{-\left(\sum_{j=1}^{17} x_{j} t_{j}+3 \tilde{t}_{1}+5 \tilde{t}_{2}\right) \beta\right\}
\end{aligned}
$$

onde, $\mathrm{D}=$ conjunto de dados observados. 
Apresentamos a seguir um resumo gráfico (Figuras 7.15, 7.16 e 7.17) e algumas estatísticas, ambos extraídos de 20000 simulações MCMC.

(a)

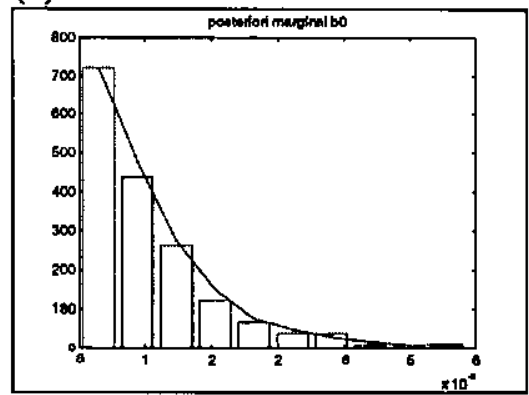

(b)

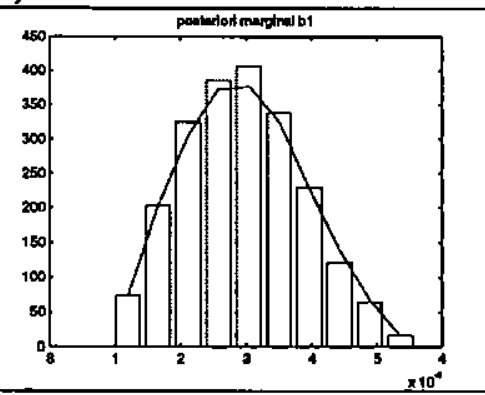

(c)

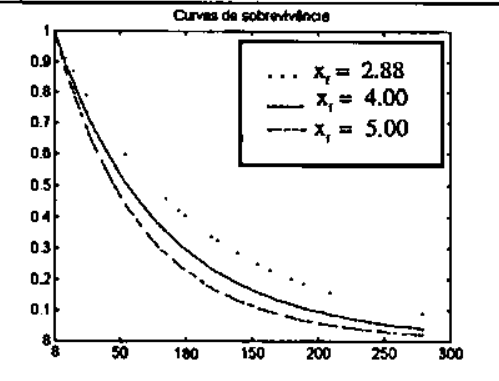

Figura 7.15 - Densidades marginais a posteriori para $\alpha$ em (a), e para $\beta$ em (b), em (c) a distribuição de sobrevivência preditiva. Ligação inversa, dados censurados e priori PDA.

(a)

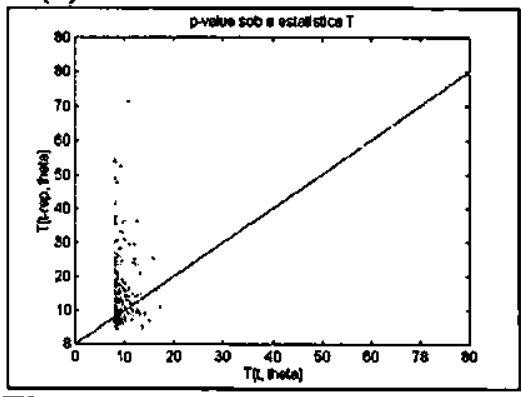

(b)

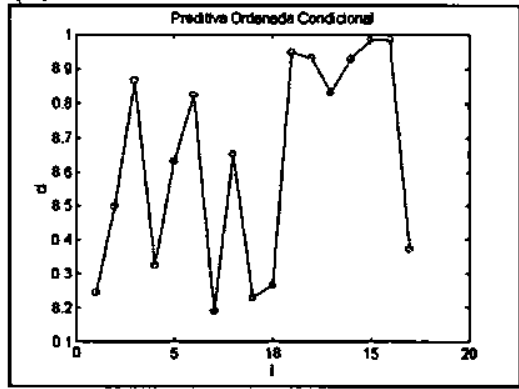

Figura 7.16 - (a) Diagrama de dispersão da estatística do teste, $\mathrm{T}(\mathrm{y}, \beta)=\sum_{\mathrm{i}} \frac{\left(\mathrm{y}_{\mathrm{i}}-\mathrm{E}\left(\mathrm{y}_{\mathrm{i}} \mid \beta\right)\right)^{2}}{\operatorname{var}\left(\mathrm{y}_{\mathrm{i}} \mid \beta\right)}$, para o nível descritivo bayesiano, $\left(\boldsymbol{n}_{d}=\mathbf{7 3 , 8} \%\right)$, (na abscissa $T(y, \beta))$ versus $\left(T\left(y^{\text {rep }}, \beta\right)\right.$ na ordenada $)$, baseado em 500 simulações. (b) Preditiva ordenada condicional ( $c_{i}$ versus $\left.i\right), \mathbf{c}\left(\mathbf{l}_{4}\right)=3,300581713724643 \times 10^{-5}$.

(a)

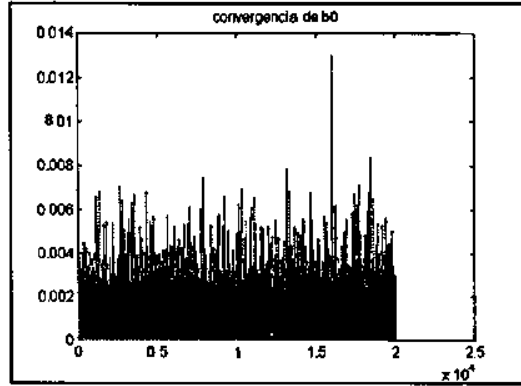

(b)

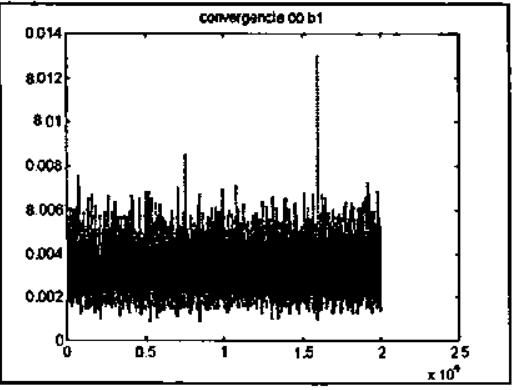

(c)

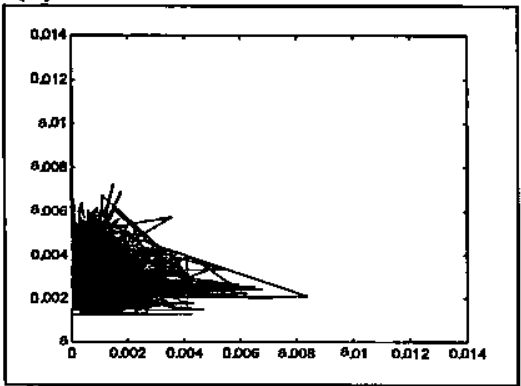

Figura 7.17 - Gráfico da convergência para os parâmetros $\alpha$ e $\beta$ em (a) $e$ (b) respectivamente, e em (c) convergência conjunta, baseados em 20000 simulaçס̃es. 
TABELA 7.7 - Estatísticas a posteriori com ligação inversa, dados censurados $e$ priori PDA.

\begin{tabular}{l|c|c}
\hline & $\alpha$ & $\beta$ \\
\hline $\mathbf{I C}(95 \%)$ & 0,$000029 ; 0,003686$ & 0,$001325 ; 0,004894$ \\
\hline $\mathbf{I C}(90 \%)$ & 0,$000051 ; 0,003034$ & 0,$001564 ; 0,004519$ \\
\hline Critério de G. e Rubin $(\sqrt{\hat{R}})$ & 1.027291 & 1.006345 \\
\hline Média & 0,0010 & 0,0030 \\
\hline Erro padrão & 0,00099 & 0,00091 \\
\hline Taxa de aceitação & $88,3 \%$ & $68,3 \%$ \\
\hline
\end{tabular}

\subsubsection{MODELO COM DADOS CENSURADOS E PRIORI GAMA}

Iremos considerar a priori conjunta sugerida por Bhattacharya et al (1995), dada pela equação (4.13) e hiperparâmetros $v=1, a=1 ; q=2$ e $b=1$.

Utilizando a verossimilhança dada por (7.10), temos as seguintes densidades marginais a posteriori:

$$
\begin{aligned}
& \pi(\alpha \mid \beta, D) \propto \prod_{i=1}^{12}\left(1+\frac{\beta}{\alpha} x_{i}\right) \alpha^{12} \exp \left[-\alpha\left(1+\sum_{j=1}^{17} t_{j}\right)\right] \\
& \pi(\beta \mid \alpha, D) \propto \prod_{i=1}^{12}\left(\frac{\alpha}{\beta}+x_{i}\right) \beta^{13} \exp \left[-\beta\left(1+\sum_{j=1}^{17} x_{j} t_{j}\right)\right] .
\end{aligned}
$$

Devemos chamar a atenção para o fato de que a parametrização $z_{i}=x_{i}-\bar{x}$, não pode ser utilizada em (7.14) e (7.15), pois produziria hiperparâmetros negativos.

A seguir, apresentamos um resumo gráfico (Figuras 7.18, 7.19 e 7.20) e algumas estatísticas, ambos extraídos de 20000 simulações MCMC.

(a)

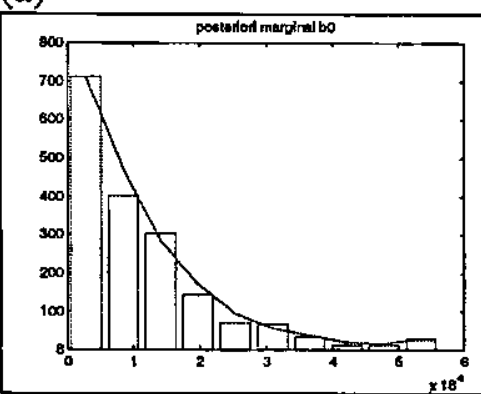

(b)

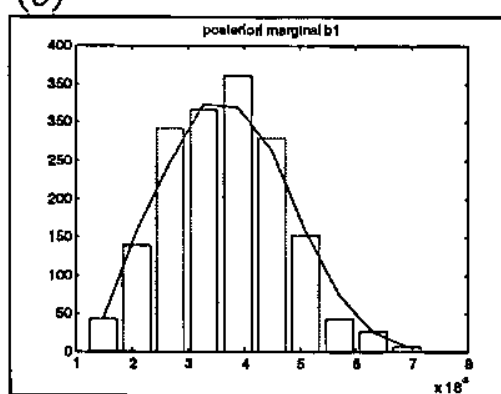

(c)

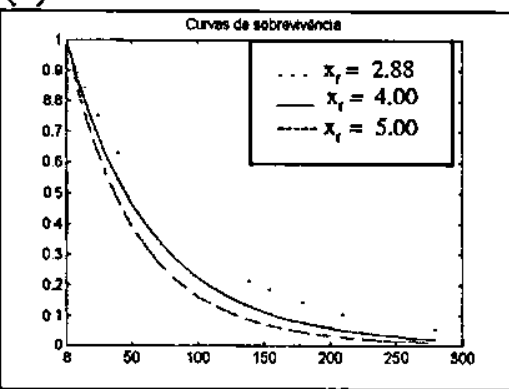

Figura 7.18 - Densidades marginais a posteriori para $\alpha$ em (a), e para $\beta$ em (b), em (c) a distribuição de sobrevivência preditiva. Ligação inversa, dados censurados e priori gama. 
(a)

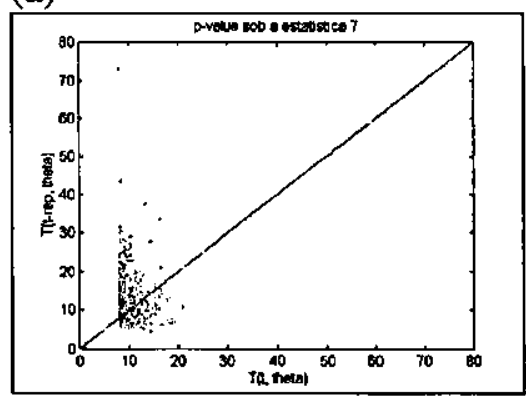

(b)

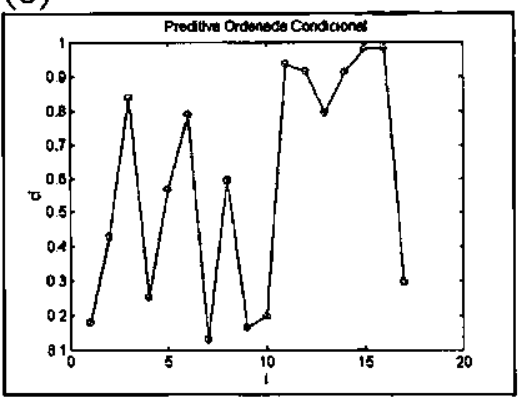

Figura 7.19 - (a) Diagrama de dispersão da estatística do teste,

$\mathrm{T}(\mathrm{y}, \beta)=\sum_{\mathrm{i}} \frac{\left(\mathrm{y}_{\mathrm{i}}-\mathrm{E}\left(\mathrm{y}_{\mathrm{i}} \mid \beta\right)\right)^{2}}{\operatorname{var}\left(\mathrm{y}_{\mathrm{i}} \mid \beta\right)}$, para o nível descritivo bayesiano, $\left(\boldsymbol{n}_{\boldsymbol{d}}=\mathbf{6 3 , 0 \%}\right)$, (na

abscissa $T(y, \beta))$ versus $\left(T\left(y^{\text {rep }}, \beta\right)\right.$ na ordenada $)$, baseado em 500 simulaçōes.

(b) Preditiva ordenada condicional $\left(c_{i}\right.$ versus $i$ ), $c\left(l_{5}\right)=3,404746777683440 \times 10^{-6}$.

(a)

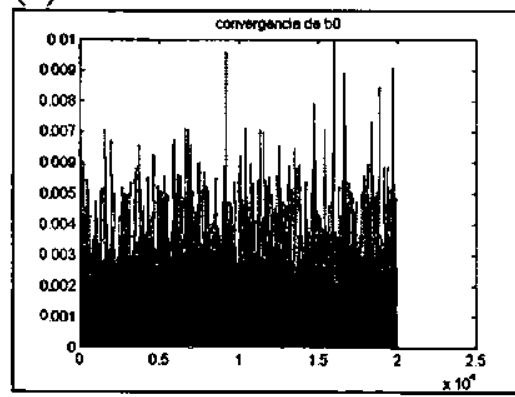

(b)

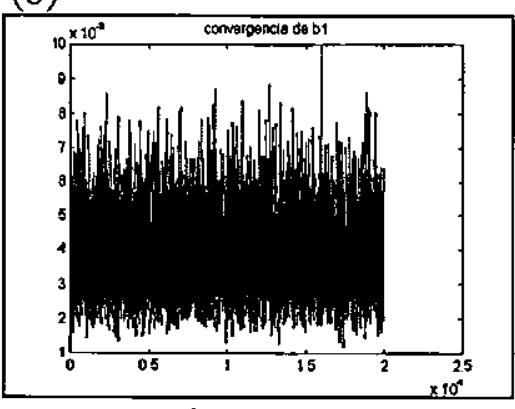

(c)

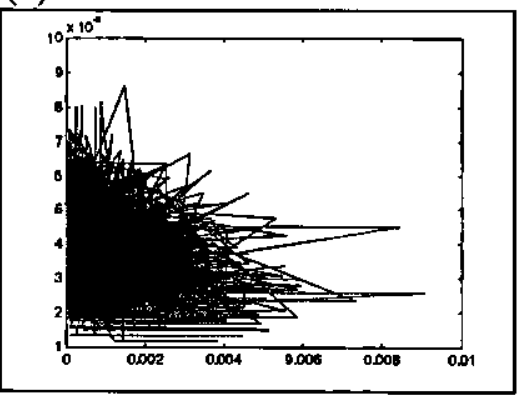

Figura 7.20 - Gráfico da convergência para os parâmetros $\alpha$ e $\beta$ em (a) $e$ (b) respectivamente, e em (c) convergência conjunta, baseados em 20000 simulações.

TABELA 7.8 - Estatísticas a posteriori com ligação inversa, dados censurados e priori gama.

\begin{tabular}{l|c|c}
\hline & $\alpha$ & $\beta$ \\
\hline $\mathrm{IC}(95 \%)$ & 0,$000031 ; 0,004140$ & 0,$001764 ; 0,005743$ \\
\hline $\mathrm{IC}(90 \%)$ & 0,$000078 ; 0,003297$ & 0,$002016 ; 0,005375$ \\
\hline Critério de G. e Rubin $(\sqrt{\hat{\mathrm{R}}})$ & 1,000416 & 1,017518 \\
\hline Média & 0,0011 & 0,0037 \\
\hline Erro padrão & 0,00107 & 0,00105 \\
\hline Taxa de aceltação & $89,6 \%$ & $71,6 \%$ \\
\hline
\end{tabular}

Uma abordagem alternativa a metodologia que usamos aqui, apresentada na seção (4.2) sob o ponto de vista da inferência bayesiana exata, é sugerida por Bhattacharya et al (1995). Para esta inferência, desenvolvemos um programa computacional (ver Apêndice C). Encontramos as 
seguintes estimativas: $\hat{\alpha}=0.00506$ e $\hat{\beta}=0.00314$, através das equações (4.18) e (4.19), respectivamente.

Podemos destacar a grande vantagem da inferência bayesiana aproximada via MCMC sobre a inferência exata, pois esta, como se pode observar ainda requer neste caso programas computacionais, que exigem uma certa dificuldade. Um outro ponto importante que podemos destacar, no caso da inferência exata desenvolvida é por exemplo, sua inviabilidade na obtenção de intervalos de credibilidade, ao contrário da obtenção via inferência aproximada.

Todos os cálculos e procedimentos feitos para o modelo com amostra censurada e priori gama também foram realizados para o modelo com a amostra completa.

\subsection{INFERÊNCIA PARA A FUNÇÃO DE LIGAÇÃO LINEAR}

Vamos concentrar nossas atenções aqui nesta seção ao modelo com priori PDA e amostra censurada, os outros casos em que abordamos até aqui, podem ser obtidos de modo semelhante.

\subsubsection{MODELO COM DADOS CENSURADOS E PRIORI PDA}

Assumiremos novamente o modelo exponencial (2.1), sob a função de ligação linear, dada em (2.2).

A função de verossimilhança pode ser escrita na seguinte forma:

$$
L(\alpha, \beta)=\prod_{i=1}^{12}\left(\alpha+\beta x_{i}\right)^{-1} \exp \left\{-\sum_{j=1}^{17} t_{j}\left(\alpha+\beta x_{j}\right)^{-1}\right\}
$$

A priori conjunta para $\alpha$ e $\beta$ é dada por:

$$
\pi(\alpha, \beta) \propto(\alpha+3 \beta)^{-2}(\alpha+5 \beta)^{-2} \exp \left\{-\tilde{t}_{1}(\alpha+3 \beta)^{-1}-\tilde{t}_{2}(\alpha+5 \beta)^{-1}\right\}
$$

Através de (7.16) e (7.17), temos a seguinte densidade a posteriori conjunta: 


$$
\begin{aligned}
\pi(\alpha, \beta \mid D) \propto & \prod_{i=1}^{12}\left(\alpha+\beta x_{i}\right)^{-1} \exp \left\{-\sum_{j=1}^{17} t_{j}\left(\alpha+\beta x_{i}\right)^{-1}\right\} . \\
& (\alpha+3 \beta)^{-2}(\alpha+5 \beta)^{-2} \exp \left\{-\tilde{t}_{1}(\alpha+3 \beta)^{-1}-\tilde{t}_{2}(\alpha+5 \beta)^{-1}\right\}
\end{aligned}
$$

onde, $\mathrm{D}=$ conjunto de dados observados, também neste caso, não reparametrizados.

Apresentamos a seguir um resumo gráfico (Figuras 7.21, 7.22 e 7.23) e algumas estatísticas, ambos extraídos de 10000 simulações MCMC.
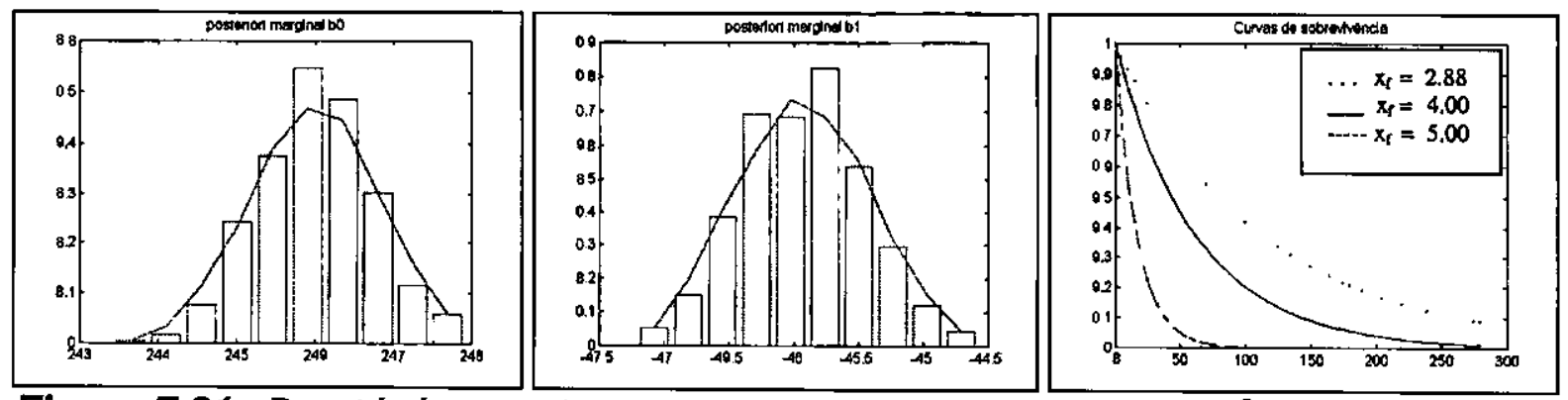

Figura 7.21 - Densidades marginais a posteriori para $\alpha$ em (a), e para $\beta$ em (b), em (c) a distribuição de sobrevivência preditiva. Ligação linear, dados censurados e priori PDA.

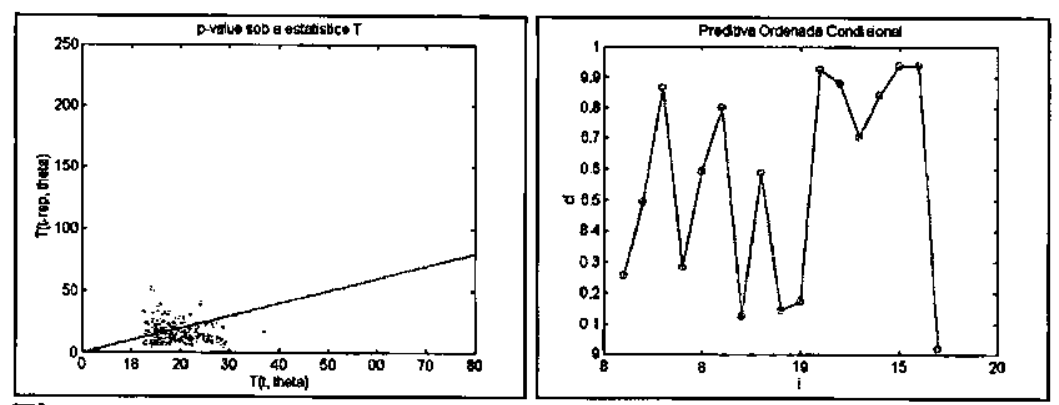

Figura 7.22 - (a) Diagrama de dispersão da estatística do teste, $\mathrm{T}(\mathrm{y}, \beta)=\sum_{\mathrm{i}} \frac{\left(\mathrm{y}_{\mathrm{i}}-\mathrm{E}\left(\mathrm{y}_{\mathrm{i}} \mid \beta\right)\right)^{2}}{\operatorname{var}\left(\mathrm{y}_{\mathrm{i}} \mid \beta\right)}$, para o ntvel descritivo bayesiano, $\left(\boldsymbol{n}_{d}=\mathbf{2 9 , 8 \%}\right),($ na abscissa $T(y, \beta))$ versus $\left(T\left(y^{\text {rep }}, \beta\right)\right.$ na ordenada $)$, baseado em 500 simulaçōes.

(b) Preditiva ordenada condicional ( $c_{i}$ versus $\left.i\right), \mathbf{c}\left(\mathrm{I}_{6}\right)=2,342892138380050 \times 10^{-7}$.
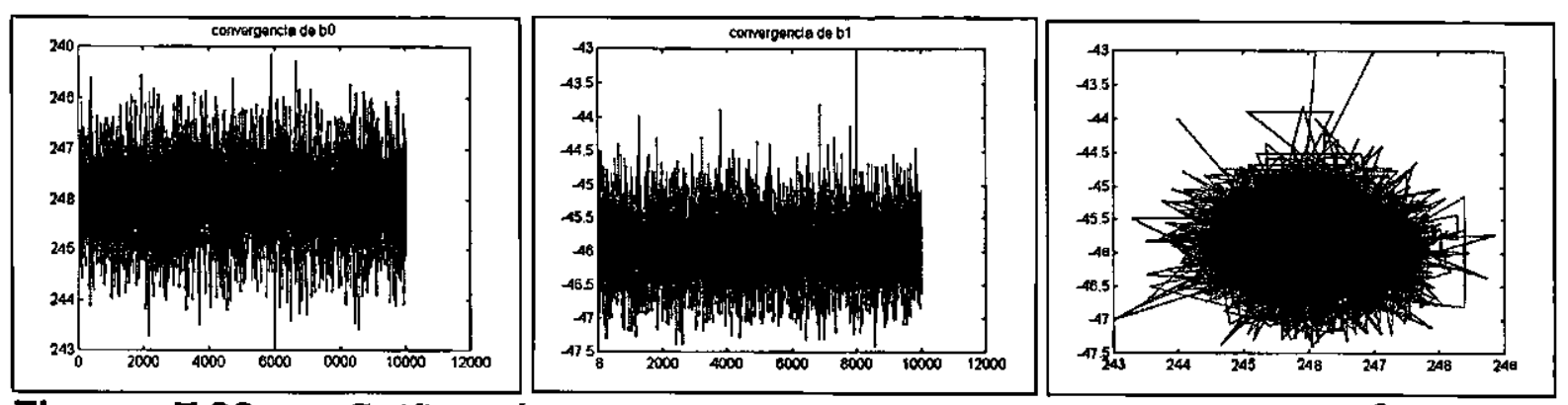

Figura 7.23 - Gráfico da convergência para os parâmetros $\alpha \quad e \quad \beta \quad e m(a) e$ (b) respectivamente, e em (c) convergência conjunta, baseados em 20000 simulações. 
TABELA 7.9 - Estatísticas a posteriori com ligação linear, dados censurados e priori PDA.

\begin{tabular}{l|c|c}
\hline & $\alpha$ & $\beta$ \\
\hline IC(95\%) & 244,$59 ; 247,48$ & $-46,82 ;-45,00$ \\
\hline IC(90\%) & 244,$84 ; 247,17$ & $-46,69 ;-45,13$ \\
\hline Critério de G. e Rubin $(\sqrt{\hat{R}})$ & 1,001156 & 1.000239 \\
\hline Média & 245,99 & $-45,92$ \\
\hline Erro padrão & 0,71875 & 0,47542 \\
\hline Taxa de aceitação & $96,8 \%$ & $87,8 \%$ \\
\hline
\end{tabular}

A inferência bayesiana desenvolvida neste capítulo permitiu analisar o nosso problema sobre vários aspectos. Podemos observar que a introdução de censuras na amostra tende a produzir previsões mais otimistas do tempo de sobrevivência dos pacientes com baixa quantidade de glóbulos brancos, quando a amostra a priori não traduz bem as reais condições do paciente.

Em todos os casos que apresentamos, o nível descritivo bayesiano $\left(\boldsymbol{n}_{d}\right)$ indicou boa adequação do modelo ajustado aos dados. Em relação a escolha da função de ligação, sob o critério da $\mathrm{CPO}$, indicamos a ligação inversa (maior $C(\ell)$, ver Tabela 7.10 a seguir) como a mais apropriada para o uso de PDA's. Para as três funções de ligações, podemos observar a grande diferença nas estimativas a posteriori de $\alpha$ e de $\beta$ e uma grande mudança nos níveis da distribuição de sobrevivência preditiva, mais notável para a ligação inversa em relação as duas outras (ver Figuras 7.3, 7.15 e 7.21). Outras considerações no uso de PDA's envolvendo dados completos e censurados, podem ser vistas em Rodrigues e Chaves (1998).

TABELA 7.10 - Estatísticas a posteriori para $\alpha$ e $\beta$ considerando as funçôes de ligações logarítmica, inversa e linear, com priori PDA e dados censurados.

\begin{tabular}{c|c|c|c|c|c|c}
\hline \multirow{2}{*}{} & \multicolumn{2}{|c|}{ Logarítmica } & \multicolumn{2}{c|}{ Inversa } & \multicolumn{2}{c}{ Linear } \\
\cline { 2 - 7 } & média & erro padrão & média & erro padrão & média & erro padrão \\
\hline$\alpha$ & 4,1126 & 0,2576 & 0,0010 & 0,00099 & 245,99 & 0,71875 \\
\hline$\beta$ & $-0,8842$ & 0,3292 & 0,0030 & 0,00091 & $-45,92$ & 0,47542 \\
\hline CPO $(C(\ell))$ & $6,384959631162401 \times 10^{-6}$ & $3,300581713724643 \times 10^{-5}$ & $2,342892138380050 \times 10^{-7}$ \\
\hline
\end{tabular}

Desejamos ainda chamar a atenção para o fato de que o uso da ligação inversa no nosso problema, produz curvas de sobrevivência preditiva muito próximas para os três níveis da QGB considerados. Isto resultaria em previsões que não iriam distinguir bem um paciente num estado grave dos outros menos graves. Portanto, sugerimos neste caso a ligação logarítmica, apesar de possuir uma $\mathrm{CPO}(\mathrm{C}(\ell))$ menor. Neste caso, se faz necessário o uso de outros critérios, para a discriminação de modelos, além da CPO. 


\section{Capítulo 8}

\section{CONCLUSÕES FINAIS E PROPOSTAS FUTURAS}

A inferência bayesiana que desenvolvemos, aproximada via métodos MCMC para analisar dados clínicos exponenciais permitiu levar em consideração as três funções de ligações propostas e densidades a priori PDA, gama e não-informativa difusa, associadas ao tempo de sobrevivência dos pacientes. Sendo uma alternativa a inferência clássica e principalmente a inferência exata. Não conseguiríamos, por exemplo, trabalhar com densidades a priori do tipo PDA sob o ponto de vista da inferência bayesiana exata em face das complicações teóricas que teríamos que enfrentar, muito além daquelas que tivemos para a priori gama.

$O$ uso da ligação inversa com priori PDA resultaria em previsões do tempo de sobrevivência que não iriam distinguir bem um paciente num estado grave, dos outros menos graves. Portanto, a ligação logarítmica, é sugerida como uma alternativa mais apropriada.

Podemos considerar a implementação de densidades a priori do tipo PDA como a principal contribuição do nosso trabalho a análise de dados clínicos exponenciais e em especial, quando estamos diante de dados censurados.

Em relação as considerações futuras, desejamos destacar:

1. Extensão para várias variáveis auxiliares dos procedimentos desenvolvidos para a inferência bayesiana via MCMC.

2. Introduzir erros nas variáveis auxiliares.

3. Necessidade de métodos alternativos a CPO para discriminação de modelos.

4. Aplicar a metodologia desenvolvida para analisar dados reais atuais. 


\section{Apêndice A}

\section{Integrais Utilizadas na Inferência Bayesiana Exata}

Representação Integral da Funcão Bessel Modificada :

$$
\int_{0}^{\infty} z^{v-1} e^{-A z^{-1}-B z} d z=2\left[\frac{A}{B}\right]^{v / 2} \cdot k_{v}(2 \sqrt{A B})
$$

$$
(\operatorname{Re} A, \operatorname{Re} B>0)
$$

onde $K_{-v}(z)=K_{v}(z)$ é a função Bessel modificada (Abramowitz e Stegun, 1965). Tem-se que :

$$
\begin{aligned}
\mathrm{K}_{\mathrm{n}}(\mathrm{z})= & \frac{1}{2}\left(\frac{1}{2} \mathrm{z}\right)^{-\mathrm{n}} \sum_{\mathrm{k}=0}^{\mathrm{n}-1} \frac{(\mathrm{n}-\mathrm{k}-1) !}{\mathrm{k} !}\left(-\frac{1}{4} \mathrm{z}^{2}\right)^{\mathrm{k}}+(-1)^{\mathrm{n}+1} \ln \left(\frac{1}{2} \mathrm{z}\right) \mathrm{J}_{\mathrm{n}}(\mathrm{z})+ \\
& +(-1)^{\mathrm{n}} \frac{1}{2}\left(\frac{1}{2} \mathrm{z}\right)^{\mathrm{n}} \sum_{\mathrm{k}=0}^{\infty}\{\psi(\mathrm{k}+1)+\psi(\mathrm{n}+\mathrm{k}+1)\} \frac{\left(\frac{1}{4} \mathrm{z}^{2}\right)^{\mathrm{k}}}{\mathrm{k} !(\mathrm{n}+\mathrm{k}) !},
\end{aligned}
$$

onde

$$
\begin{aligned}
& J_{n}(z)=\left(\frac{1}{2} z\right)^{n} \sum_{k=0}^{\infty} \frac{\left(\frac{1}{4} z^{2}\right)^{k}}{k ! \Gamma(n+k+1)}, \\
& \psi(1)=-\gamma=-0.577215664901532860606512 \ldots, \\
& \psi(n)=-\gamma+\sum_{k=1}^{n-1} k^{-1}, \quad(n \geq 2) .
\end{aligned}
$$

Integral para a função Whittaker :

$$
\int_{u}^{\infty} x^{v-1}(x-u)^{A-1} e^{-B x} d x=B^{-\left(\frac{A+v}{2}\right)} u^{\left(\frac{A+v-2}{2}\right)} \Gamma(A) e^{-\frac{B u}{2}} \cdot \frac{W_{v-A}}{2} \frac{1-A-v}{2}(B u)
$$


onde, $\operatorname{Re} \mathrm{A}>0, \operatorname{Re} \mathrm{Bu}>0, \mathrm{e} \mathrm{W}_{\mathrm{k}, \mu}($ ) é a função Whittaker (Abramowitz e Stegun, 1965). Tem-se que :

$$
\begin{aligned}
& W_{k, \mu}(z)=e^{-\frac{1}{2} z} z^{\frac{1}{2}+\mu} U\left(\frac{1}{2}+\mu-k, 1+2 \mu, z\right), \\
&\left(-\pi<\arg z \leq \pi, k=\frac{1}{2} b-a, \mu=\frac{1}{2} b-\frac{1}{2}\right),
\end{aligned}
$$

onde

$$
\mathrm{U}(\mathrm{a}, \mathrm{b}, \mathrm{c})=\frac{\pi}{\operatorname{sen} \pi \mathrm{b}} \cdot\left\{\frac{\mathrm{M}(\mathrm{a}, \mathrm{b}, \mathrm{z})}{\Gamma(1+\mathrm{a}-\mathrm{b}) \Gamma(\mathrm{b})}-\mathrm{z}^{1-b} \frac{\mathrm{M}(1+\mathrm{a}-\mathrm{b}, 2-\mathrm{b}, \mathrm{z})}{\Gamma(\mathrm{a}) \cdot \Gamma(2-b)}\right\},
$$

com $\mathrm{M}(\mathrm{a}, \mathrm{b}, \mathrm{z})$ dada pela função Kummer (Abramowitz e Stegun, 1965) :

$$
\begin{aligned}
& M(a, b, z)=1+\frac{a z}{b}+\frac{(a)_{2} z^{2}}{(b)_{2} 2 !}+\cdots+\frac{(a)_{n} z^{n}}{(b)_{n} n !}+\cdots \\
& (a)_{n}=a(a+1)(a+2) \cdots(a+n-1) ; \quad(a)_{0}=1 .
\end{aligned}
$$

A representação integral (A.2) pode ser transformada por uma simples substituição $\mathrm{x}-\mathrm{u}=\mathrm{t}$ para obtermos o seguinte resultado:

$$
\begin{aligned}
& \int_{0}^{\infty}(t+u)^{v-1} t^{A-1} e^{-B t} d t=B{ }^{-\left(\frac{A+v}{2}\right)} u^{\left(\frac{A+v-2}{2}\right)} \Gamma(A) e^{\frac{B u}{2}} \cdot \frac{W-A}{2}, \frac{1-A-v}{2}(B u) \\
& \text { onde } \operatorname{Re} A>0, \operatorname{Re} B u>0 .
\end{aligned}
$$

Para o cálculo de uma integral envolvendo a função Whittaker deveremos utilizar :

$$
\begin{aligned}
\int_{0}^{\infty} e^{-s t} t^{\tau} \mathrm{w}_{\lambda, \delta}(\rho \mathrm{t}) \mathrm{dt}= & \frac{\Gamma(\tau+\delta+3 / 2) \Gamma(\tau-\delta+3 / 2)}{\Gamma(\tau-\lambda+2)} \rho{ }^{\delta+1 / 2}\left[\mathrm{~s}+\frac{\rho}{2}\right]^{-\tau-\delta-3 / 2} . \\
& \cdot{ }_{2} \mathrm{~F}_{1}\left[\tau+\delta+3 / 2, \delta-\lambda+1 / 2 ; \tau-\lambda+2 ; \frac{2 s-\rho}{2 s+\rho}\right]
\end{aligned}
$$

onde $: \operatorname{Re}(\tau+\delta+3 / 2)>0, \operatorname{Re} s>-\frac{\rho}{2}, \rho>0 \quad ;$

${ }_{2} F_{1}(., \ldots$, ; . ) é a função Gauss hipergeométrica (Abramowitz e Stegun, 1965), isto é :

$$
F(a, b ; c ; z)={ }_{2} F_{1}(a, b ; c ; z)=\frac{\Gamma(c)}{\Gamma(a) \Gamma(b)} \cdot \sum_{n=0}^{\infty} \frac{\Gamma(a+n) \Gamma(b+n)}{\Gamma(c+n)} \cdot \frac{z^{n}}{n !},
$$

$\operatorname{Re}(c-a-b)>0$. 


\section{Apêndice B}

\section{Simulação Monte Carlo em Cadeia de Markov (MCMC)}

Apresentamos a seguir dois métodos de simulação MCMC. Os métodos consistem na substituição da expressão analítica da densidade por uma amostra gerada a partir desta densidade. Em inferência bayesiana aplicada, este procedimento é extremamente importante devido a complexidade das distribuições a posteriori em muitas situações.

\section{B.1 O Amostrador de Gibbs}

O amostrador de Gibbs (ver por exemplo Casella e George, 1992) é uma técnica de simulação de observações de uma distribuição, sem que se tenha de calcular analiticamente a densidade. Sob o enfoque bayesiano, é um esquema iterativo de amostragem de uma cadeia de Markov, cujo núcleo de transição (ou função geradora) é formado pelas densidades condicionais completas.

Seja $\pi(\theta)$ a densidade que estamos interessados e supostamente difícil de amostrar, onde $\theta=\left(\theta_{1}, \theta_{2}, \ldots, \theta_{k}\right)$ é o vetor de parâmetros de interesse. Se as densidades a posteriori condicionais $\pi\left(\theta_{i} \mid \theta_{(i)}, D\right)$, onde $\theta_{(i)}=\left(\theta_{1}, \ldots, \theta_{i-1}, \theta_{i+1}, \ldots \theta_{k}\right)$ estão disponíveis sob uma forma padronizada, o algoritmo do amostrador de Gibbs é descrito da seguinte forma:

(1) Escolher valores iniciais para $\theta, \theta^{(0)}=\left(\theta_{1}^{(0)}, \ldots, \theta_{\mathrm{k}}^{0}\right)$ e inicialize-se o contador de interações da cadeia em $\mathrm{j}=1$; 
(2) Obter os novos valores $\theta^{(j)}=\left(\theta_{1}^{(j)}, \ldots, \theta_{k}^{j}\right)$ a partir de $\theta^{j-1}$ através de sucessivas gerações de valores:

$$
\begin{gathered}
\theta_{1}^{(j)} \sim \pi\left(\theta_{1} \backslash \theta_{2}^{(j-1)}, \ldots, \theta_{k}^{(j-1)}, D\right) \\
\theta_{2}^{(j)} \sim \pi\left(\theta_{2} \backslash \theta_{1}^{(j)}, \theta_{3}^{(j-1)} \ldots, \theta_{k}^{(j-1)}, D\right) \\
\cdot \\
\cdot \\
\cdot \\
\theta_{k}^{(j)} \sim \pi\left(\theta_{k} \backslash \theta_{1}^{(j)}, \theta_{2}^{(j)} \ldots, \theta_{k-1}^{(j)}, D\right)
\end{gathered}
$$

(3) Mudar o contador de j para j+1 e faça o retorno a (2) até obter a convergência.

\section{B.2 Algoritmo de Metropolis-Hastings}

Quando as distribuições condicionais usadas no amostrador de Gibbs não possuem formas padronizadas, devemos utilizar o algoritmo de Metropolis-Hastings (ver por exemplo, Chib e Greenberg, 1995).

Suponha que desejamos amostras da densidade $\pi\left(\theta_{\mathrm{i}} \mid \theta_{(\mathrm{i})}, \mathrm{D}\right)$, onde

$$
\theta_{(\mathrm{i})}=\left(\theta_{1}, \ldots, \theta_{\mathrm{i}-1}, \theta_{\mathrm{i}+1}, \ldots \theta_{\mathrm{k}}\right)
$$

Definir um núcleo de transição $q\left(\theta, \theta^{*}\right)$ da distribuição $\pi(\theta)$ que representa $\pi\left(\theta_{\mathrm{i}} \mid \theta_{(\mathrm{i})}, \mathrm{D}\right)$, e que transforma $\theta$ em $\theta^{*}$. Geralmente o processo se move de $\theta$ para $\theta^{*}$ com mais frequência do que de $\theta^{*}$ para $\theta$ violando a condição de reversibilidade da cadeia, isto é 


$$
\pi(\theta) \mathrm{q}\left(\theta, \theta^{*}\right)>\pi\left(\theta^{*}\right) \mathrm{q}\left(\theta^{*}, \theta\right)
$$

Este fato pode ser eliminado diminuindo o número de movimentos de $\theta$ para $\theta^{*}$, introduzindo a probabilidade de ocorrer este movimento $\alpha\left(\theta, \theta^{*}\right)<1$. Se o movimento ocorre, então $\theta^{(j+1)}=\theta^{*}$, caso contrário $\theta^{(j+1)}=\theta^{(k)}$.

O algoritmo de Metropolis-Hastings, pode ser descrito da seguinte forma:

(1) Inicialize com um valor arbitrário $\theta^{(0)}$ para $\theta$;

(2) Gerar $\theta^{*}$ de $q\left(\theta^{(j)}, \theta^{*}\right)$ e u segundo, $\mathfrak{u} \sim U[0,1]$;

(3) Seja $\alpha=\min \left\{1, \frac{\pi\left(\theta^{*}\right) \mathrm{q}\left(\theta^{*}, \theta^{(j)}\right)}{\pi\left(\theta^{(j)}\right) \mathrm{q}\left(\theta^{(j)}, \theta^{*}\right)}\right\}$. Se $u \leq \alpha$ faça $\theta^{(\mathrm{j}+1)}=\theta^{*}$, senão faça $\theta^{(\mathrm{j}+1)}=\theta^{(\mathrm{j})}$;

(4) Repetir os estágios (2) e (3) até que uma distribuição estacionária tenha sido obtida.

A escolha de $q\left(\theta, \theta^{*}\right)$ pode ser feita de várias maneiras:

(a) Quando a posteriori é escrita na forma $\pi(\theta) \propto \phi(\theta) h(\theta)$, onde $h(\theta)$ é uma densidade que pode ser amostrada e $\phi(\theta)$ limitada, tomamos $h\left(\theta^{*}\right)=q\left(\theta, \theta^{*}\right)$ como candidato gerador. Neste caso :

$$
\alpha=\min \left\{1, \frac{\phi\left(\theta^{*}\right)}{\phi\left(\theta^{(j)}\right)}\right\}
$$

(b) $\mathrm{q}\left(\theta, \theta^{*}\right)=\mathrm{q}_{1}\left(\theta-\theta^{*}\right)$, onde $\mathrm{q}_{1}($.$) é uma densidade multivariada. Neste caso, \theta^{*}=\theta+\varepsilon$, onde $\varepsilon$ é a variável incremento com distribuição $\mathrm{q}_{1}($.$) . Se \mathrm{q}_{1}(\varepsilon)=\mathrm{q}_{1}(-\varepsilon)$, então segundo Muiller (1993): 


$$
\alpha=\min \left\{1, \frac{\pi\left(\theta^{*}\right)}{\pi\left(\theta^{(j)}\right)}\right\} .
$$

Para outras formas alternativas na escolha de $q(.,$.$) e os respectivos cuidados nesta$ escolha, ver Chib e Greenberg (1995). Por exemplo, se q(.,.) apresentar muita variabilidade isto implicará em convergência lenta ou baixa taxa de aceitação.

Após a convergência, todas as cadeias devem ter o mesmo comportamento e, supondo que isso aconteça na $k$-ésima iteração, usam-se os valores $\theta_{j}, \theta_{j+h}, \theta_{j+2 h}, \ldots, \theta_{j+n h}$ onde $j \geq k$, como uma amostra aleatória da distribuição desejada. Para que essa amostra seja i.i.d, h tem que ser um número suficientemente grande, tal que permita haver independência entre as realizaçōes.

Vários critérios para verificar a convergência são propostos na literatura. Neste trabalho utilizamos o critério de Gelman e Rubin (1992), o qual é baseado em técnicas de análise de variância, onde a convergência é aceita quando a variância entre as cadeias for bem menor do que a variância dentro de cada cadeia. 


\section{Apêndice C}

\section{ALGUNS PROGRAMAS DESENVOLVIDOS}

A seguir, apresentamos alguns programas que desenvolvemos em MATLAB utilizados nas várias aplicações realizadas.

Programa para o modelo com priori PDA e função de ligação logarítmica. Dados com censuras.

$\% \quad$ Modelo com Priori informativa (PDA) em MLG e amostra censurada

$\% \quad$ via Algoritmo de Metropolis-Hastings

$\% \quad$...............Ligação logarítmica

$\%$ Dados de pacientes com leucemia aguda (Zippin e armitage, 1966)

$\% \quad$ Preditiva Condicional Ordenada,

$\%$ densidades marginais a posteriori, estatísticas, funçao de sobrevivencia.

clear

t0 =clock;

$\% \quad$ Conjunto de dados

format long e

qgb $=\left[\begin{array}{ll}7502300260043005400600070009400100001050017000 \ldots\end{array}\right.$

$320003500052000100000100000100000]$;

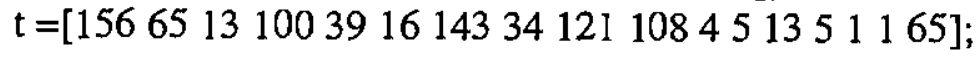

qgbc $=\left[\begin{array}{lllllllll}7502300 & 4300 & 540060007000 & 10000 & 10500 & 17000 & 52000 & 100000 & 100000\end{array}\right] ;$

tc $=\left[\begin{array}{llllllllllll}156 & 65 & 100 & 39 & 16 & 143 & 121 & 108 & 4 & 5 & 1 & 65\end{array}\right]$

$\%$......... (qgbc,tc) $=$ parte não-censurada $;(\mathrm{qgb}, \mathrm{t})=$ amostra completa

$\mathrm{x} 0=\log 10$ (qgb); $\mathrm{n}=17 ; \mathrm{d}=12$;

$\mathrm{x} 0 \mathrm{c}=\log 10(\mathrm{qgbc})$;

$\mathrm{m}=$ mean $(\mathrm{x} 0)$;

$\mathrm{x}=\mathrm{x} 0-\mathrm{m} ; \mathrm{xc}=\mathrm{x} 0 \mathrm{c}-\mathrm{m} ; \%$ (reparametrização)

$\mathrm{n} 1=3-\mathrm{m} ; \mathrm{n} 2=5-\mathrm{m} ; \quad \%$ (níveis da QGB)

$\%$ condiçōes iniciais:

$\mathrm{v}=\left[\begin{array}{llllll}-5 & 4 & 8 & 12 & 5\end{array}\right.$

$-10-5-1-.75]$; 
iter $=10000$;

cont $1=0$; cont $2=0$;

for $\mathrm{k}=2$ :iter+1

if rem(k-2,2000) $==0$ \& $\mathrm{k}<$ iter

$\mathrm{b} 0(\mathrm{k}-1)=\mathrm{v}(1,1+(\mathrm{k}-2) / 2000)$;

$\mathrm{bl}(\mathrm{k}-1)=\mathrm{v}(2,1+(\mathrm{k}-2) / 2000)$;

end

\% geração de b0

$\mathrm{b} 0 \mathrm{v}=\mathrm{b} 0(\mathrm{k}-1) ; \mathrm{b} 1 \mathrm{v}=\mathrm{b} 1(\mathrm{k}-1)$;

$\%$

calculo dos parametros do nucleo

$\%$ gerando candidato $\mathrm{p} / \mathrm{b} 0$

$\mathrm{a}=4 ; \mathrm{b}=.6 ; \quad \% \mathrm{EMV} \mathrm{b} 0=4.14$

y0 =normmd $(a, b)$;

\% teste para aceitação de y0 como novo valor da var. b0

$$
\begin{aligned}
& \text { for } j=1: n \\
& \text { e1a(j) }=\exp \left(-\left(y 0+b 1 v^{*} x(j)\right)\right) \text {; } \\
& \text { end }
\end{aligned}
$$

el $=-t^{*} e 1 a^{\prime} ;$

$\mathrm{e} 2=-86.37^{*} \exp (-(\mathrm{y} 0+\mathrm{n} 1 * \mathrm{~b} 1 \mathrm{v}))$;

e $3=-19.04 * \exp (-(y 0+n 2 * b 1 v))$;

$\% \operatorname{psin} 1=\exp (-(\mathrm{d}+2) * \mathrm{y} 0) * \exp (\mathrm{e} 1+\mathrm{e} 2+\mathrm{e} 3) ;($ numerador $)$

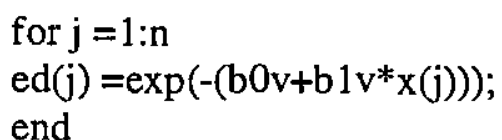

eld $=-t^{*}$ ed';

e2d $=-86.37 * \exp (-(b 0 v+n 1 * b 1 v))$;

e3d $=-19.04 * \exp (-(b 0 v+n 2 * b 1 v))$

$\%$ psidl $=\exp (-(\mathrm{d}+2) * \mathrm{~b} 0 \mathrm{v}) * \exp (\mathrm{e} 1 \mathrm{~d}+\mathrm{e} 2 \mathrm{~d}+\mathrm{e} 3 \mathrm{~d})$; (denominador $)$

psi $1=\exp (-(\mathrm{d}+2) * \mathrm{y} 0+\mathrm{e} 1+\mathrm{e} 2+\mathrm{e} 3+(\mathrm{d}+2) * \mathrm{~b} 0 \mathrm{v}-\mathrm{e} 1 \mathrm{~d}-\mathrm{e} 2 \mathrm{~d}-\mathrm{e} 3 \mathrm{~d})$;

prob $1=\min (1, \mathrm{psi} 1)$

$\mathrm{U}=$ rand;

if $\mathrm{U}<=$ prob1

$\mathrm{b} 0(\mathrm{k})=\mathrm{y} 0$;

cont $1=$ cont $1+1$;

else

$\mathrm{b} 0(\mathrm{k})=\mathrm{b} 0(\mathrm{k}-1)$;

end

$\%$ fim do teste

$\mathrm{bOn}=\mathrm{bO}(\mathrm{k})$;

\% geração de b1 
$\%$ calculo dos parametros do nucleo

$\% \quad$ gerando candidato $\mathrm{p} / \mathrm{b} 1$

$\mathrm{a}=-1 ; \mathrm{b}=.5 ; \quad \%$ EMV $\mathrm{bl}=-0.80$

$\mathrm{y} 1=$ normrnd(a,b);

$\%$ teste para aceitação de y 1 como novo valor da var. b1

for $\mathrm{j}=1: \mathrm{n}$

$\mathrm{e} 1 \mathrm{a}(\mathrm{j})=\exp (-(\mathrm{b} 0 \mathrm{n}+\mathrm{y} 1 * \mathrm{x}(\mathrm{j})))$;

end

e1 $=-t^{*}$ el $a^{\prime}$;

$\mathrm{e} 2=-86.37 * \exp (-(\mathrm{b} 0 \mathrm{n}+\mathrm{n} 1 * \mathrm{y} 1))$;

e $3=-19.04 * \exp (-(\mathrm{bOn}+\mathrm{n} 2 * \mathrm{y} 1))$

$\% \operatorname{psin} 2=\exp (-(\operatorname{sum}(\mathrm{xc})+\mathrm{n} 1+\mathrm{n} 2) * \mathrm{y} 1) * \exp (\mathrm{e} 1+\mathrm{e} 2+\mathrm{e} 3) ;($ numerador $)$

for $\mathrm{j}=1: n$

$\operatorname{ed}(j)=\exp \left(-\left(b 0 n+b 1 v^{*} x(j)\right)\right)$;

end

eld $=-$ - $^{*}$ ed';

e2d $=-86.37 * \exp (-(b 0 n+n 1 * b 1 v))$;

e3d $=-19.04 * \exp (-(b 0 n+n 2 * b 1 v))$;

$\%$ psid $2=\exp \left(-(\operatorname{sum}(\mathrm{xc})+\mathrm{n} 1+\mathrm{n} 2)^{*} \mathrm{~b} 1 \mathrm{v}\right) * \exp (\mathrm{e} 1 \mathrm{~d}+\mathrm{e} 2 \mathrm{~d}+\mathrm{e} 3 \mathrm{~d}) ;($ denominador $)$

psi2 $=\exp (-(\operatorname{sum}(x c)+n 1+n 2) * y 1+e 1+e 2+e 3+(\operatorname{sum}(x c)+n 1+n 2) * b 1 v-e 1 d-e 2 d-e 3 d)$

$\operatorname{prob} 2=\min (1, \mathrm{psi} 2)$;

$\mathrm{U}=\mathrm{rand}$;

if $\mathrm{U}<=$ prob2

$\mathrm{b} 1(\mathrm{k})=\mathrm{y} 1$;

cont $2=$ cont $2+1$;

else

$\mathrm{b} 1(\mathrm{k})=\mathrm{b} 1(\mathrm{k}-1)$;

end

$\%$ fim do teste

end

\% Seleção das amostras finais

$\mathrm{ks}=1$;

cont $=0$;

for $\mathrm{j}=501: 2000$ :iter;

for $\mathrm{i}=\mathrm{j}: \mathrm{j}+1499$;

if $\operatorname{rem}(i-j+1,15)==0$

$\mathrm{b} 0 \mathrm{~s}(\mathrm{ks})=\mathrm{bO}(\mathrm{i})$;

$\mathrm{b} 1 \mathrm{~s}(\mathrm{ks})=\mathrm{b} 1(\mathrm{i})$;

\%....... cálculo do nivel descritivo bayesiano 


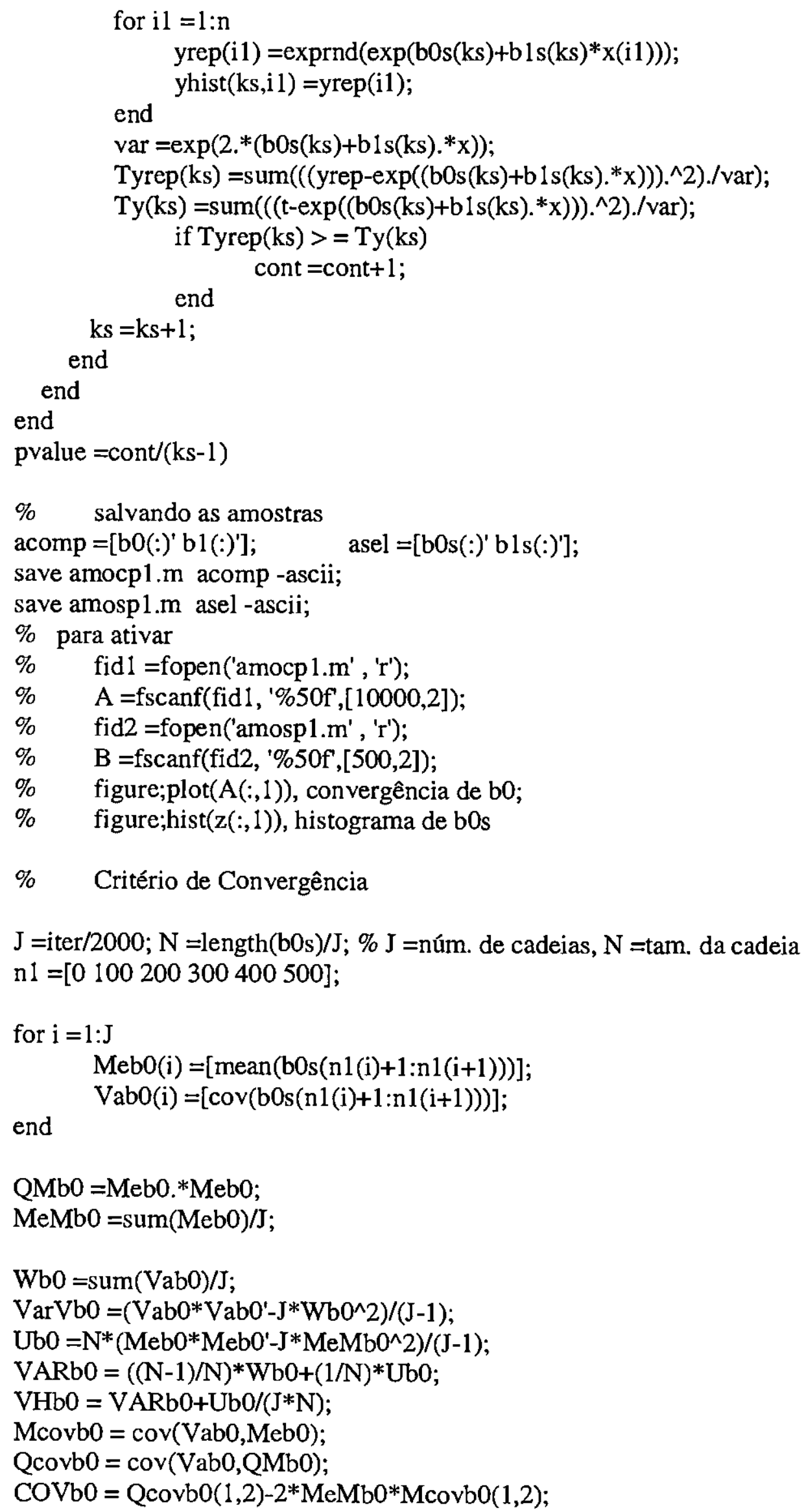




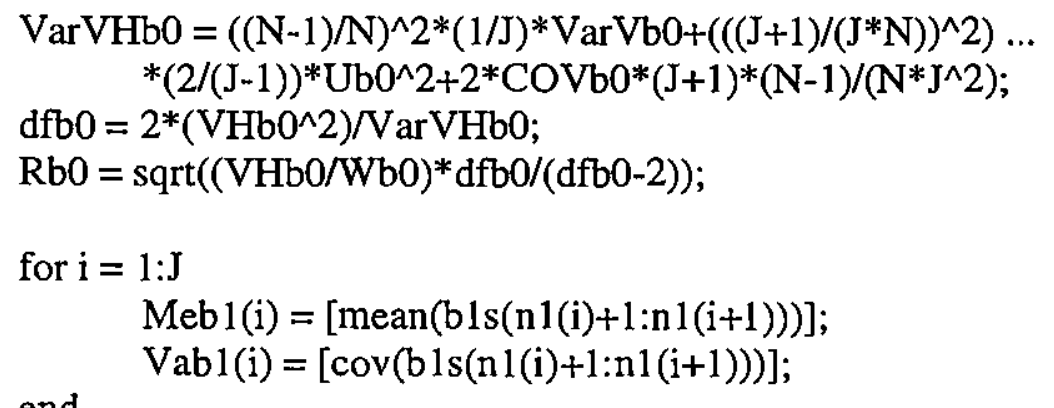

QMb1=Meb1.*Meb1;

$\mathrm{MeMb} 1=\operatorname{sum}(\mathrm{Meb} 1) / \mathrm{J}$;

$\mathrm{Wb} 1=\operatorname{sum}(\operatorname{Vab} 1) / \mathrm{J}$;

$\operatorname{VarVb} 1=\left(\operatorname{Vab} 1^{*} \operatorname{Vab} 1^{\prime}-J^{*} * \mathrm{~Wb} 1^{\wedge} 2\right) /(J-1)$;

$\mathrm{Ub} 1=\mathrm{N} *\left(\mathrm{Meb} 1 * \mathrm{Meb} 1{ }^{\prime}-\mathrm{J} * \mathrm{MeMb} 1^{\wedge} 2\right) /(\mathrm{J}-1)$;

$\mathrm{VARb} 1=((\mathrm{N}-1) / \mathrm{N}) * \mathrm{~Wb} 1+(1 / \mathrm{N}) * \mathrm{Ub} 1$;

$\mathrm{VHb} 1=\mathrm{VARb} 1+\mathrm{Ub} 1 /(\mathrm{J} * \mathrm{~N})$;

Mcovb1=cov(Vab1,Meb1);

Qcovb1=cov(Vab1,QMb1);

COVb1=Qcovb 1 $(1,2)-2 * M e M b 1 * M \operatorname{covb} 1(1,2)$;

$\operatorname{VarVHb} 1=((\mathrm{N}-1) / \mathrm{N})^{\wedge} 2 *(1 / \mathrm{J}) * \operatorname{VarVb} 1+\left(((\mathrm{J}+1) /(\mathrm{J} * \mathrm{~N}))^{\wedge} 2\right)^{*} \ldots$ $(2 /(\mathrm{J}-1))^{*} \mathrm{Ub} 1^{\wedge} 2+2 * \mathrm{COVb}^{*}(\mathrm{~J}+1)^{*}(\mathrm{~N}-1) /\left(\mathrm{N}^{*} \mathrm{~J}^{\wedge} 2\right)$;

$\mathrm{dfb} 1=2 *\left(\mathrm{VHb} 1^{\wedge} 2\right) / \mathrm{VarVHb} 1$;

$\mathrm{Rb} 1=\operatorname{sqrt}((\mathrm{VHb} 1 / \mathrm{Wb} 1) * \mathrm{dfb} 1 /(\mathrm{dfb} 1-2))$;

disp(['Critério de Convergência de Rubin para b0 e b1: ']);

$\mathrm{R}=[\mathrm{Rb} 0 ; \mathrm{Rb} 1]$

namostra $=\mathrm{ks}-1$

$\% \quad$ ESTATÍSTICAS

'media e desvio'

$\mathrm{B} 0=[\operatorname{mean}(\mathrm{b} 0 \mathrm{~s}) \operatorname{std}(\mathrm{b} 0 \mathrm{~s})]$

$\mathrm{B} 1=[$ mean $(\mathrm{b} 1 \mathrm{~s}) \quad \mathrm{std}(\mathrm{b} 1 \mathrm{~s})]$

ACEITACAOb0 $=$ cont $1 /$ iter

ACEITACAOb $1=$ cont $2 /$ iter

$\% \quad$ intervalos de credibilidade

$P=[2.552550759597 .5]^{\prime}$, percb0=prctile(b0s, $\left.\mathrm{P}\right)$, percb1=prctile(b1s, $\left.\mathrm{P}\right)$

$\%$ Graficos

figure; whitebg('w')

histfit2('smo',10,b0s)

\%title('marginal posterior of a')

figure 
histfit2('smo',10,b1s)

\%title('marginal posterior of $b^{\prime}$ )

figure

plot(b0,b1)

figure

plot(b0)

title('convergencia de b0')

figure

plot(b1)

title('convergencia de b1')

figure;hist(yhist(:))

title('histograma das 500 réplicas')

figure; hist(t)

title( 'histograma do tempo de vida')

figure

plot(Ty, Tyrep, 'b.')

hold on

$i=0: 1: 80 ; j=0: 1: 80$;

$\operatorname{plot}\left(\mathrm{i}, \mathrm{j}, \mathrm{I}^{\prime}\right)$

xlabel('T(t, theta)')

ylabel('T(t-rep, theta)')

title(' nivel descritivo bayesiano sob a estatistica T')

$\%$

Preditiva Ordenada Condicional

for $\mathrm{j}=1: n$

$x f=[1 x(j)]$;

for $\mathrm{i}=1$ :namostra

$\mathrm{b}=[\mathrm{b} 0 \mathrm{~s}(\mathrm{i}) \mathrm{bls}(\mathrm{i})]$;

end

$\operatorname{predt}(\mathrm{i})=\exp \left(-\mathrm{t}(\mathrm{j}) \cdot{ }^{*} \exp \left(-\mathrm{xf}^{*} \mathrm{~b}^{\prime}\right)\right)$;

end

$c(j)=$ mean (predt);

$\mathrm{cl}=\operatorname{prod}(\mathrm{c})$

figure

$\mathrm{i}=1: 1: \mathrm{n}$;

plot(i,c,'b-',i,c,'ro')

title('Preditiva Ordenada Condicional')

xlabel('i')

ylabel('ci')

\% função de sobrevivência preditiva

ts $=0: 10: 280$

$\mathrm{vf}=\left[\begin{array}{lll}2.88 & 4 & 5\end{array}\right]$;

for $\mathrm{j}=1$ :length(vf) 


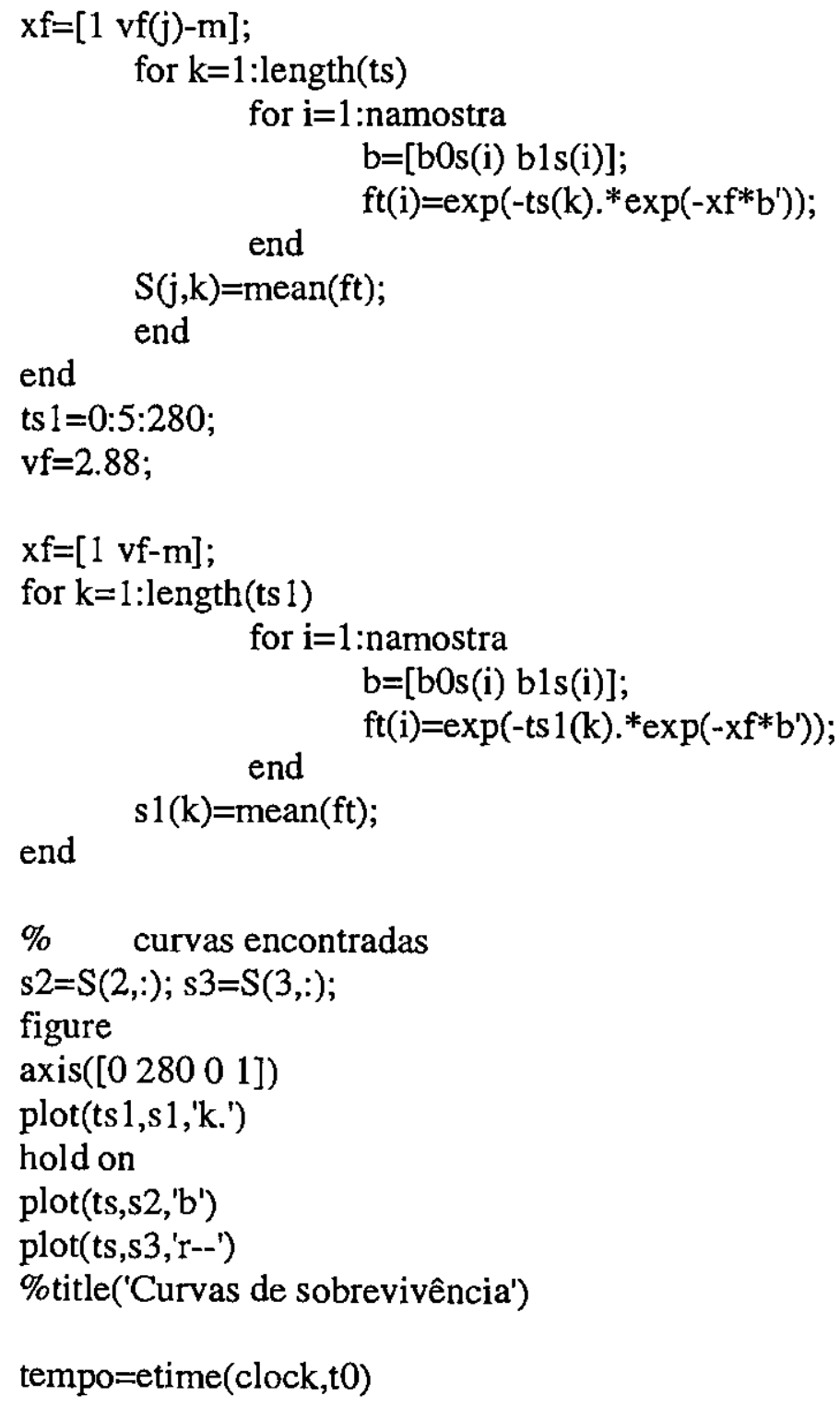


Programa para inferência bayesiana exata com ligação inversa e amostra com censuras.

clear

format long e

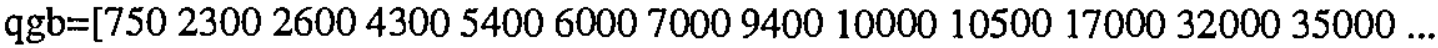
$52000100000100000100000]$;

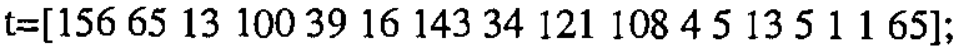

$\mathrm{x} 0=\log 10$ (qgb); $\mathrm{n}=17$;

$\% \times 0=x 0$-mean $(\mathrm{x} 0) ; \%$ (reparametrização)

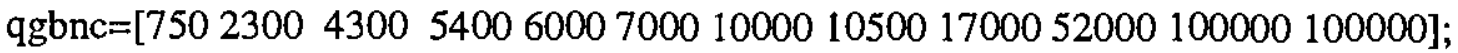

tnc $=\left[\begin{array}{llllllllllll}156 & 65 & 100 & 39 & 16 & 143 & 121 & 108 & 4 & 5 & 1 & 65\end{array}\right]$;

$\%$... (qgbnc,tnc) $=$ parte não-censurada; $(q g b, t)=$ amostra completa

$\mathrm{d}=12$;

$\mathrm{xnc}=\log 10$ (qgbnc); $\mathrm{x}=\mathrm{xnc}$;

$\mathrm{xreg}=\left[\mathrm{ones}(\mathrm{n}, 1) \times 0^{\prime}\right]$;

$\mathrm{T}=\mathrm{t} . \wedge(-1) ; \quad \%$..... Ligação Inversa .....

\%bil $=$ regress $\left(\mathrm{T}^{\prime}, \mathrm{xreg}\right)$

bi2 $=\left(\operatorname{inv}\left(x^{\prime r e g}{ }^{\prime *} x r e g\right)\right) *\left(x^{\prime} g^{\prime *} T^{\prime}\right)$;

$\% b^{\wedge}=\left[\begin{array}{ll}.1761 & .3171\end{array}\right]$

\% Calculo dos hiperparâmetros $(\mathrm{v}, \mathrm{a}) \mathrm{e}(\mathrm{q}, \mathrm{b})$ da priori conjunta

\%aest $=.1761$; best $=.3171$;

$\% \operatorname{var} 1=.01 ; \operatorname{var} 2=.01 ;$

$\% \mathrm{a}=$ aest/var 1 ; $=$ =aest*a; $\quad \mathrm{b}=$ best/var $2 ; \mathrm{q}=$ best*b;

$\mathrm{a}=1 ; \mathrm{v}=1 ; \mathrm{q}=2 ; \mathrm{b}=1$;

$\%$ Produto dos $\mathrm{r}$ elementos distintos do conjunto $\{\mathrm{x} 1, \ldots, \mathrm{x} 12\}$

$\%$ Cálculo da constante $\mathrm{K}$ e das estimativas a posteriori de

$\%$ alfa e beta

$\mathrm{S}=\operatorname{sum}(\mathrm{t}) ; \quad \mathrm{Q}=\operatorname{sum}(\mathrm{x} 0 . * \mathrm{t})$

$\mathrm{s} 0=1$

$s 1=\operatorname{sum}(x)$

$N=$ length $(x)$;

$s 2=0 ;$

for $\mathrm{i}=1: \mathrm{N}-1$

end

$s 2=\operatorname{sum}(x(i) * x(i+1: N))+s 2 ;$

for $i 1=1: N-2$ 
for $i 2=i 1+1: N-1$

$\mathrm{s} 3=\operatorname{sum}(x(\mathrm{i} 1) * x(\mathrm{i} 2) * x(\mathrm{i} 2+1: \mathrm{N}))+\mathrm{s} 3$;

end

end

$\mathrm{s} 4=0$

for $\mathrm{i} 1=1: \mathrm{N}-3$

for $\mathrm{i} 2=\mathrm{i} 1+1: \mathrm{N}-2$

for $i 3=i 2+1: N-1$

$s 4=\operatorname{sum}\left(x(\mathrm{i} 1)^{*} x(\mathrm{i} 2)^{*} x(\mathrm{i} 3)^{*} x(\mathrm{i} 3+1: N)\right)+s 4 ;$

end

end

end

s5 $=0$;

for $\mathrm{i} 1=1: \mathrm{N}-4$

for $\mathrm{i} 2=\mathrm{i} 1+1: \mathrm{N}-3$

for $\mathrm{i} 3=\mathrm{i} 2+1: \mathrm{N}-2$

for $\mathrm{i} 4=\mathrm{i} 3+1: \mathrm{N}-1$

$\mathrm{s} 5=\operatorname{sum}\left(x(\mathrm{i} 1)^{*} \mathrm{x}(\mathrm{i} 2) * x(\mathrm{i} 3)^{*} \mathrm{x}(\mathrm{i} 4)^{*} \mathrm{x}(\mathrm{i} 4+1: \mathrm{N})\right)+\mathrm{s} 5$; end

end

end

end

s6=0;

for $i 1=1: N-5$

for $i 2=i 1+1: N-4$

for $i 3=i 2+1: N-3$

for $i 4=i 3+1: N-2$

for $\mathrm{i} 5=\mathrm{i} 4+1: \mathrm{N}-1$

$\mathrm{s} 6=\operatorname{sum}\left(x(\mathrm{i} 1)^{*} x(\mathrm{i} 2)^{*} x(\mathrm{i} 3)^{*} x(\mathrm{i} 4) * x(\mathrm{i} 5) * x(\mathrm{i} 5+1: \mathrm{N})\right)+\mathrm{s} 6$;

end

end

end

end

end

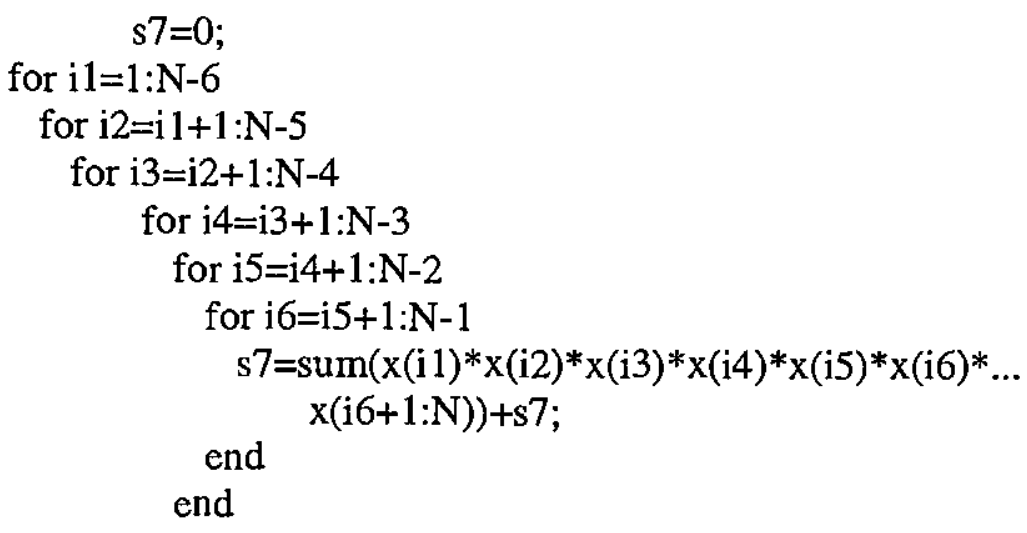




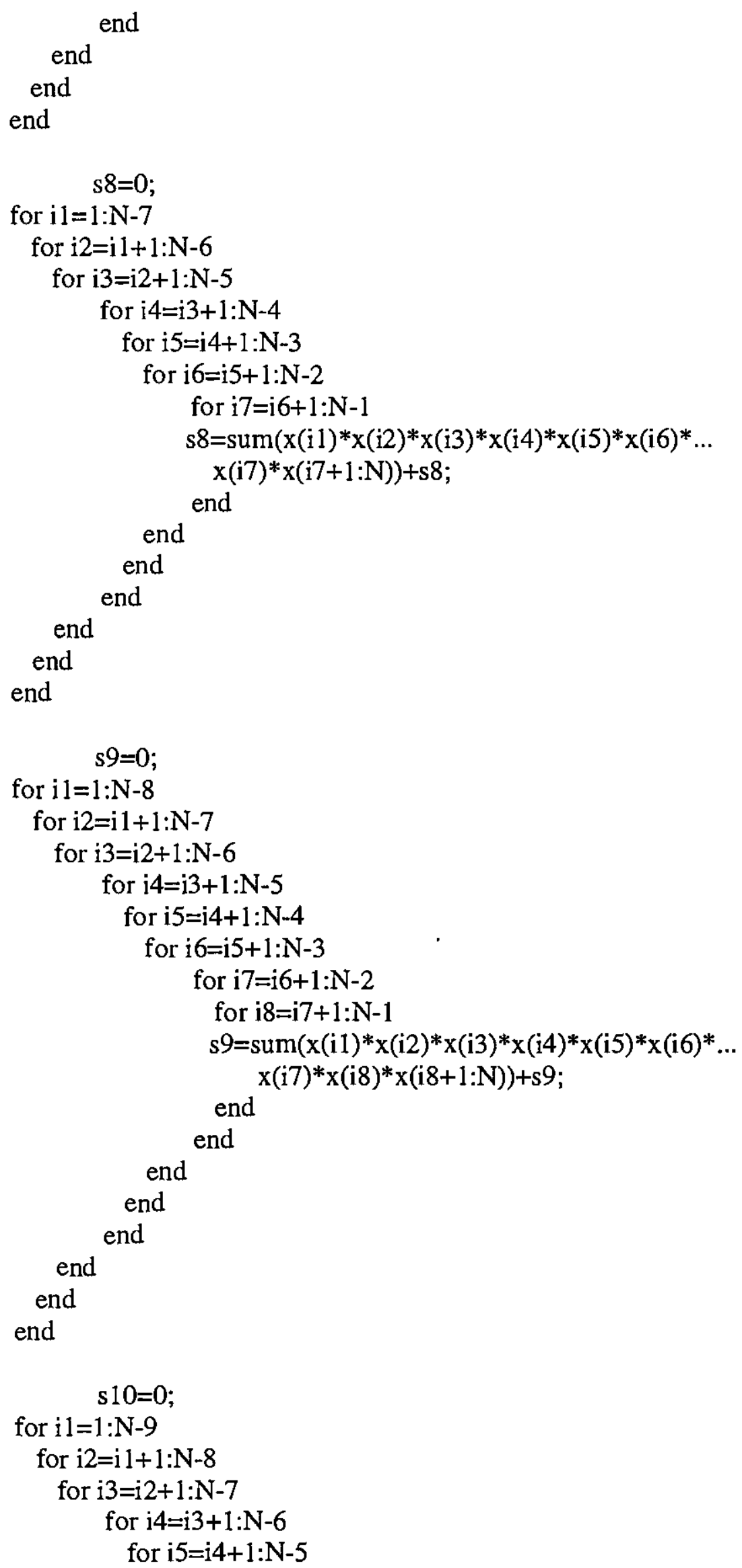



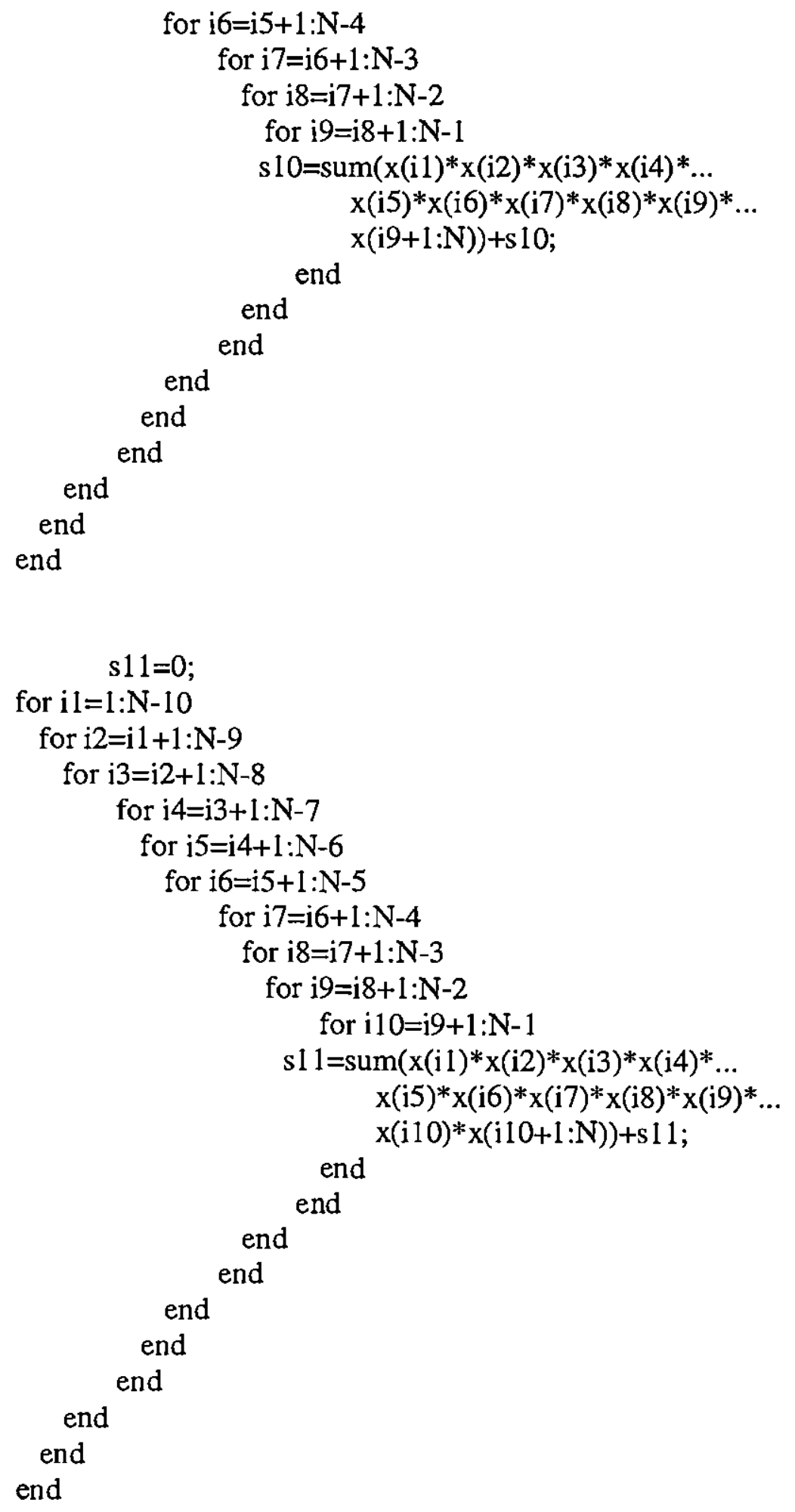

s12= $\operatorname{prod}(x) ;$

$\%$ soma $=\mathrm{s} 0+\mathrm{s} 1+\mathrm{s} 2+\mathrm{s} 3+\mathrm{s} 4+\mathrm{s} 5+\mathrm{s} 6+\mathrm{s} 7+\mathrm{s} 8+\mathrm{s} 9+\mathrm{s} 10+\mathrm{s} 11+\mathrm{s} 12$;

$\mathrm{srd}=[\mathrm{s} 0 \mathrm{~s} 1 \mathrm{~s} 2 \mathrm{~s} 3 \mathrm{~s} 4 \mathrm{~s} 5 \mathrm{~s} 6 \mathrm{~s} 7 \mathrm{~s} 8 \mathrm{~s} 9 \mathrm{~s} 10 \mathrm{~s} 11 \mathrm{~s} 12]$ 
\% Cálculo da constante $\mathrm{k}$

$\mathrm{r}=0 ; \mathrm{k}=0$;

for $\mathrm{i}=1$ :length(srd)

$\mathrm{k}=\mathrm{k}+\operatorname{srd}(\mathrm{i})^{*}\left(\operatorname{gamma}(\mathrm{d}+\mathrm{v}-\mathrm{r})^{*} \operatorname{gamma}(\mathrm{q}+\mathrm{r})\right) /\left(\left((\mathrm{a}+\mathrm{S})^{\wedge}(\mathrm{d}+\mathrm{v}-\mathrm{r})\right)^{*}(\mathrm{~b}+\mathrm{Q})^{\wedge}(\mathrm{q}+\mathrm{r})\right) ;$

$\mathrm{r}=\mathrm{r}+1$;

end

$\% \quad$ Estimativa de alfa

$\mathrm{r}=0 ; \mathrm{S} 1=0$;

for $\mathrm{i}=1$ :length(srd)

$\mathrm{S} 1=\mathrm{S} 1+\operatorname{srd}(\mathrm{i}) *\left(\operatorname{gamma}(\mathrm{d}+\mathrm{v}-\mathrm{r}+1)^{*} \operatorname{gamma}(\mathrm{q}+\mathrm{r})\right) /\left(\left((\mathrm{a}+\mathrm{S})^{\wedge}(\mathrm{d}+\mathrm{v}-\mathrm{r}+1)\right)^{*}(\mathrm{~b}+\mathrm{Q})^{\wedge}(\mathrm{q}+\mathrm{r})\right)$; $\mathrm{r}=\mathrm{r}+1$;

end

alfa $=\left(\mathrm{k}^{\wedge}(-1)\right)^{*} \mathrm{~S} 1$

$\% \quad$ estimativa de beta

$\mathrm{r}=0 ; \mathrm{S} 2=0$;

for $\mathrm{i}=1$ : length(srd)

$\mathrm{S} 2=\mathrm{S} 2+\operatorname{srd}(\mathrm{i})^{*}\left(\operatorname{gamma}(\mathrm{d}+\mathrm{v}-\mathrm{r})^{*} \operatorname{gamma}(\mathrm{q}+\mathrm{r}+1)\right) /\left(\left((\mathrm{a}+\mathrm{S})^{\wedge}(\mathrm{d}+\mathrm{v}-\mathrm{r})\right)^{*}(\mathrm{~b}+\mathrm{Q})^{\wedge}(\mathrm{q}+\mathrm{r}+1)\right)$; $\mathrm{r}=\mathrm{r}+1$;

end

beta $=\left(k^{\wedge}(-1)\right)^{*} \mathrm{~S} 2$

\% calculo da variancia de alfa e beta

$\mathrm{r}=0 ; \mathrm{S} 3=0$;

for $\mathrm{i}=1$ :length(srd)

$\mathrm{S} 3=\mathrm{S} 3+\operatorname{srd}(\mathrm{i}) *\left(\operatorname{gamma}(\mathrm{d}+\mathrm{v}-\mathrm{r}+2)^{*} \operatorname{gamma}(\mathrm{q}+\mathrm{r})\right) /\left(\left((\mathrm{a}+\mathrm{S})^{\wedge}(\mathrm{d}+\mathrm{v}-\mathrm{r}+2)\right)^{*}(\mathrm{~b}+\mathrm{Q})^{\wedge}(\mathrm{q}+\mathrm{r})\right)$; $r=r+1$;

end

valfa $=\left(\mathrm{k}^{\wedge}(-1)\right)^{*} \mathrm{~S} 3-(\text { alfa })^{\wedge}(2)$

$\mathrm{r}=0 ; \mathrm{S} 4=0$;

for $\mathrm{i}=1$ :length(srd)

$\mathrm{S} 4=\mathrm{S} 4+\operatorname{srd}(\mathrm{i}) *\left(\operatorname{gamma}(\mathrm{d}+\mathrm{v}-\mathrm{r})^{*} \operatorname{gamma}(\mathrm{q}+\mathrm{r}+2)\right) /\left(\left((\mathrm{a}+\mathrm{S})^{\wedge}(\mathrm{d}+\mathrm{v}-\mathrm{r})\right)^{*}(\mathrm{~b}+\mathrm{Q})^{\wedge}(\mathrm{q}+\mathrm{r}+2)\right)$; $\mathrm{r}=\mathrm{r}+1$;

end

vbeta $=\left(\mathrm{k}^{\wedge}(-1)\right)^{*} \mathrm{~S} 4-(\text { beta })^{\wedge}(2)$ 
Programa para a inferência clássica com ligação logarítmica e dados com censuras.

clear

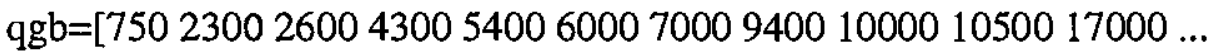

$320003500052000100000100000100000]$;

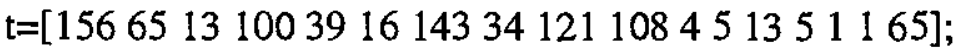

qgbc=[lf50 2300 430054006000700010000105001700052000100000100000$]$;

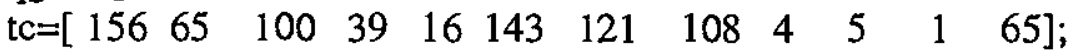

$\mathrm{x} 0=\log 10(\mathrm{qgb}) ; \mathrm{n}=17 ; \mathrm{d}=12 ;$

$\mathrm{xOc}=\log 10(\mathrm{qgbc})$;

$\mathrm{m}=\operatorname{mean}(\mathrm{x} 0)$;

$\mathrm{x}=\mathrm{x} 0-\mathrm{m} ; \mathrm{xc}=\mathrm{x} 0 \mathrm{c}-\mathrm{m} ; \%$ (reparametrização)

$\mathrm{n} 1=3-\mathrm{m} ; \mathrm{n} 2=5-\mathrm{m} ; \quad \%$ (níveis da QGB)

\% estimativas de b0 e b1 via N. Raphson :

$\%$ bOe $=4.136543726180247 \mathrm{e}+000 ;$ ble=-7.988470536172783e-001;

$\%$ condições iniciais

$\mathrm{a}(1)=3 ; \mathrm{b}(1)=-1$;

iter=100;

for $\mathrm{k}=2$ :iter

$\mathrm{f} 1(\mathrm{k})=-12+\operatorname{sum}(\mathrm{t} . * \exp (-(\mathrm{a}(\mathrm{k}-1)+\mathrm{b}(\mathrm{k}-1) * \mathrm{x})))$

$\mathrm{f} 2(\mathrm{k})=\operatorname{sum}\left(\left(\mathrm{x} \cdot{ }^{*} \mathrm{t}\right) \cdot{ }^{*} \exp \left(-\left(\mathrm{a}(\mathrm{k}-1)+\mathrm{b}(\mathrm{k}-1)^{*} \mathrm{x}\right)\right)\right)-\operatorname{sum}(\mathrm{xc})$;

$\% \quad$ Derivadas

df $1 \mathrm{a}=-\operatorname{sum}\left(\mathrm{t} . * \exp \left(-\left(\mathrm{a}(\mathrm{k}-1)+\mathrm{b}(\mathrm{k}-1)^{*} \mathrm{x}\right)\right)\right)$

$\mathrm{df} 1 \mathrm{~b}=-\operatorname{sum}\left(\left(\mathrm{x} .{ }^{*} \mathrm{t}\right){ }^{*} \exp \left(-\left(\mathrm{a}(\mathrm{k}-1)+\mathrm{b}(\mathrm{k}-1)^{*} \mathrm{x}\right)\right)\right)$;

df $2 a=d f I b ;$

df $2 b=-\operatorname{sum}\left(\left(t *^{*}\left(x .^{\wedge}(2)\right)\right) *^{*} \exp \left(-\left(a(k-1)+b(k-1)^{*} x\right)\right)\right)$;

$\mathrm{J}=[\mathrm{df} 1 \mathrm{a}$ df $1 \mathrm{~b}$

df2a df2b];

$\mathrm{I}=\operatorname{inv}(\mathrm{J})$;

$\mathrm{j} 11=I(1,1) ; \mathrm{j} 12=\mathrm{I}(1,2) ; \mathrm{j} 21=\mathrm{I}(2,1) ; \mathrm{j} 22=\mathrm{I}(2,2)$;

$\mathrm{a}(\mathrm{k})=\mathrm{a}(\mathrm{k}-1)-(\mathrm{j} 11 * \mathrm{f} 1(\mathrm{k})+\mathrm{j} 12 * \mathrm{f} 2(\mathrm{k}))$;

end

$b(k)=b(k-1)-(j 21 * f 1(k)+j 22 * f 2(k))$;

$\mathrm{bOe}=\mathrm{a}$ (iter)

$\mathrm{ble}=\mathrm{b}(\mathrm{iter})$ 
$A=d f 1 a ; \quad B=d f 2 b ; \quad C=d f 1 b$

$\%$ erros-padroes assintoticos de b0 e b1 :

epb0 $=\operatorname{sqrt}\left(-\mathrm{B} /\left(\mathrm{A}^{*} \mathrm{~B}-\mathrm{C}^{\wedge} 2\right)\right), \quad \%$ ou epb0 $=\operatorname{sqrt}(-\mathrm{I}(1,1))$ epb1=sqrt $\left(-A /\left(A^{*} B-C^{\wedge} 2\right)\right), \quad \%$ ou epb1 $=\operatorname{sqrt}(-I(2,2))$

$\% \quad$ Intervalos de confianca $90 \%$ e $95 \%$ :

$\mathrm{p} 1=.95 ; \mathrm{p} 2=0.975$

$\mathrm{z} 1=\operatorname{norminv}(\mathrm{p} 1,0,1) ; \mathrm{z} 2=\operatorname{norminv}(\mathrm{p} 2,0,1)$;

li $90 \mathrm{~b} 0=b 0 \mathrm{e}-\mathrm{z} 1 * \mathrm{epb0} ;$ ls $90 \mathrm{b0}=\mathrm{b} 0 \mathrm{e}+\mathrm{zl}$ *epb0;

li $90 \mathrm{~b} 1=\mathrm{b} 1 \mathrm{e}-\mathrm{z} 1{ }^{*} \mathrm{epb} 1 ;$ Is $90 \mathrm{~b} 1=\mathrm{b} 1 \mathrm{e}+\mathrm{z} 1 * \mathrm{epb} 1$;

li $95 b 0=b 0 e-z 2 *$ epb0; Is $95 b 0=b 0 e+z 2 * e p b 0$; li $95 \mathrm{~b} 1=\mathrm{b} 1 \mathrm{e}-\mathrm{z} 2 * \mathrm{epb} 1$; ls95b1=b1e+z2*epb1;

IC $90 \mathrm{bo}=[\mathrm{li} 90 \mathrm{b0}$ ls $90 \mathrm{b0}]$

IC $95 b 0=[1$ i $95 b 0$ ls $95 b 0]$

IC $90 \mathrm{~b} 1=[1 \mathrm{i} 90 \mathrm{~b} 1 \mathrm{ls} 90 \mathrm{~b} 1]$

$\mathrm{IC} 95 \mathrm{~b} 1=[\mathrm{li} 95 \mathrm{~b} 1 \mathrm{ls} 95 \mathrm{~b} 1]$

$\%$ verossimilança relativa

$\mathrm{b} 0 \mathrm{i}=3 ; \mathrm{b} 0 \mathrm{~s}=5.1 ; \mathrm{bli}=-3 ; \mathrm{b} 1 \mathrm{~s}=.7$;

$\mathrm{ib} 0=(\mathrm{b} 0 \mathrm{~s}-\mathrm{b} 0 \mathrm{i}) / 50 ; \mathrm{ib} 1=(\mathrm{bls}-\mathrm{bli}) / 50$;

b0=b0i:ib0:b0s; b1=b1i:ib1:b1s;

for $\mathrm{i}=1$ :length(b1)

for $\mathrm{j}=1$ :length(b0)

e1=exp(-d*b0(j)-(b1(i))*sum(xc));

for $\mathrm{k}=1: \mathrm{n}$

$e 2 a(k)=\exp (-(b 0(j)+b 1(i) * x(k))) ;$

end

$e 2=-t^{*} e 2 a^{\prime} ;$

$L(i, j)=e 1^{*} \exp (e 2)$;

end

end

$\mathrm{Lmax}=\max (\max (\mathrm{L}))$;

cont $=0$;

if $L \max ==0$

cont $=$ cont +1 ; 


\author{
else \\ $\mathrm{L}=\mathrm{L}$ './Lmax; \\ end
}

figure; whitebg('w')

$\operatorname{mesh}(\mathrm{b} 1, \mathrm{~b} 0, \mathrm{~L})$

axis([1.02*b1i b1s $0.98 * b 0 i$ b0s 0 l])

$\% \operatorname{axis}\left(\left[\begin{array}{llllll}-2.2 & 0 & 2.9 & 5 & 0 & 1\end{array}\right]\right)$

xlabel('b1')

ylabel('b0')

\%zlabel('verossimilhança(b0,b1)')

$\%$ contornos

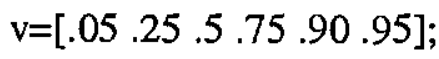

figure

contour(bl,b0,L,v)

xlabel('b1')

ylabel('b0')

\% função de sobrevivência preditiva

$\mathrm{ti}=0: 5: 280$;

$\mathrm{vf}=\left[\begin{array}{ll}2.88 & 4\end{array}\right]$;

for $\mathrm{j}=1$ :length(vf)

$x f=[1 v f(j)-m]$;

for $\mathrm{k}=1$ :length(ti)

$\mathrm{b}=[\mathrm{b} 0 \mathrm{e}$ ble];

end

$S(j, k)=\exp \left(-t i(k) \cdot * \exp \left(-x f^{*} b^{\prime}\right)\right)$;

end

$\% \quad$ Curvas encontradas

$\mathrm{s} 1=\mathrm{S}(1, \mathrm{)}) ; \mathrm{s} 2=\mathrm{S}(2,:) ; \mathrm{s} 3=\mathrm{S}(3,:) ;$

figure

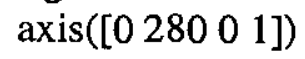

plot(ti,s1,'k.')

hold on

plot(ti,s2,'b')

plot(ti,s3,'r--')

title('Curvas de sobrevivência') 


\section{Referências Bibliográficas}

ABRAMOWITZ, M. e Stegun, I. A. (1965). Handbook of Mathematical Functions with Formulas, Graphs, and Mathematical Tables. National Bureau of Standards, Applied n. 436 , Theory and Methods, Mathematics Series 55.

BEDRICK, J. E., Christensen, R. e Johnson, W. (1996). A New Perspective on Priors for Generalized Linear Models. Journal of the American Statistical Association, v. 91, p. 14501460 .

BEDRICK , J.E., Christensen, R. e Johnson, W. (1997). Bayesian Binomial Regression : Predicting Survival at a Trauma Center. The American Statistician, v. 51, n. 3, p. 211-218.

BHATTACHARYA, S. K. (1967). Bayesian Approach to Life Testing and Reliability Estimation. Journal of the American Statistical Association, v. 62, n. 317, p. 48-62.

BHATTACHARYA, S. K., Kumar, D. e Singh, N. K. (1995). Bayesian Survival Analysis of Clinical Data Using a Covariate. Revista Brasileira de Probabilidade e Estatística, v.2, p.141156.

BICKEL, P.J. e Doksum, K. A. (1977). Mathematical-Statistics Basic Ideas and Selected Topics. San Francisco: Holden-Day.

BYAR, D. P., Huse, R. e Bailar, J. C. III, and the Veterans Administration Cooperative Urological Research Group. (1974). An Exponential Model Relating Censored Survival Data and Concomitant Information for Prostatic Cancer Patients. Journal of the National Cancer Institute, v. 52, n. 2, p. 321-326.

CASELLA, G., e George, E. (1992). Explaining the Gibbs sampler. The American Statistician, v. 46, p. $167-174$.

CARLIN, B. P. e Louis, T. A. (1997). Bayes and Empirical Bayes Methods for Data Analysis. London: Chapman and Hall.

CHIB, S. e Greenberg, E. (1995). Understanding the Metropolis-Hastings Algorithm. The American Statistician, v. 49, n. 4, p. 327-335.

COX, D. R. (1964). Some Applications of Exponential Ordered Scores. Journal of the Royal Statistical Society, B, 26, p. 103-110.

FEIGL, P. e Zelen, M. (1965). Estimation Exponential Survival Probabilities With Concomitant Information. Biometrics, 21, p. 826-838. 
GELFAND, A.E. e Dey, D. K. (1994). Bayesian Model Choice: Asymptotic and Exact Calculations. Journal of the Royal Statistical Society, B, 56, p.501-514.

GELMAN, A. e Rubin, D.B. (1992). Inference from Iterative Simulation Using Multiple Sequences. Statistical Science, v. 7, n. 4, p. 457-511.

GELMAN, A., Carlin, J., Stern, H., e Rubin, D.B. (1995). Bayesian Data Analysis. London: Chapman and Hall.

KALBFLEISCH, J. D. e Prentice, R. L. (1980). The Statistical Analysis of Failure Time Data. New York: Wiley.

LEE, E. T. (1992). Statistical Methods for Survival Data Analysis. New York: Wiley.

MCCULLAGH, P. e Nelder, J. A. (1989). Generalized Linear Models. London: Chapman and Hall.

MÜLLER, P. (1993). A Generic Approach to Posterior Integration and Gibbs Sampling. Technical Report, Department of Statistics, Purdue University.

NELDER, J. A. e Wedderburn, R. W. M. (1972). Generalized Linear Models. Journal of the Royal Statistical Society, A, 135, 370-384.

PRENTICE, R. L. (1973). Exponential Survival with Censoring and Explanatory Variables. Biometrika, 60, 2, p. 279-288.

RODRIGUES, J. e Chaves, J. S. (1998). A Note on Bayesian Exponential Regression Model with Censored Data. Notas do Instituto de Ciências Matemáticas e de Computação, Universidade de São Paulo, n. 56. (Submetido para publicação no Journal of Applied Statistical Science, EUA).

ZIPPIN, C. e Armitage, P. (1966). Use of Concomitant Variables and Incomplete Survival Information in the Estimation of an Exponential Survival Parameter. Biometrics, 22, p. 665672. 